\title{
APPLICATION OF PHASE CHANGE MATERIAL IN SUSTAINABLE COOLING OF DATA CENTERS
}

by

\author{
NIKHIL DHIMAN \\ Presented to the Faculty of the Graduate School of \\ The University of Texas at Arlington in Partial Fulfillment \\ of the Requirements \\ for the Degree of \\ MASTER OF SCIENCE IN MECHANICAL ENGINEERING
}

THE UNIVERSITY OF TEXAS AT ARLINGTON

MAY 2013 
Copyright @ Nikhil Dhiman 2013

All Rights Reserved 


\section{ACKNOWLEDGEMENTS}

I would like to express my thanks to my advisor Prof. Agonafer for his great mentorship and support in my research in every possible ways at The University of Texas at Arlington. I am highly grateful to him for providing me both academic and industrial level research experience. Without his persistent guidance and motivation this dissertation would not have been possible

I extend my warm thanks and welcome to my committee members, Prof. Haji Sheikh and Prof. Kent Lawrence for serving my committee. I would also like to thanks Mr. Mike Kaler, Mr. James Hoverson and Mr. Naveen Kannan of Mestex - A Division of Mestek, Inc. for their consistent expert advice. Their industrial experience acquires a huge role in the completion of my research work.

Many thanks to Ms. Sally Thomson for her great help throughout the period of my association with UTA. I am obliged to thank the entire EMNSPC team and special mention to Betsegaw Gebrehiwot and Jeet Shah for their full support and guidance.

Finally, this acknowledgement would not be complete without mentioning my family and friends for their great support and encouragement.

April 12, 2013 


\section{ABSTRACT \\ APPLICATION OF PHASE CHANGE MATERIAL IN SUSTAINABLE COOLING OF DATA CENTERS}

Nikhil Dhiman, M.S.

The University of Texas at Arlington, 2013

Supervising Professor: Dr. Dereje Agonafer

Keywords: mPCM (Micro-encapsulated Phase Change Material), S.E.M (Scanning Electron Microscope)

The ever increasing information technology heat load and data center cooling energy are the main reasons to investigate the performance of microencapsulated phase change slurry over other heat transfer fluids. In recent years, more effort is being made on the development of a new technique to use the phase change materials as pump-able heat transfer fluid and as heat storage system.

Microencapsulated phase change slurry is dispersion where the phase change material, microencapsulated by a polymeric capsule, is dispersed in water. Compared to water, these new fluids have a higher heat capacity during phase change and a possible enhancement, as a result of this phase change, in the heat transfer phenomenon. The composition of phase change material used in slurry greatly affects its efficiency, If not selected properly it can cause serious damage, e.g. agglomeration and clogging of pipes. Current available systems use microencapsulated phase change slurry with heat exchangers.

The main objective of this work is to develop standalone pump-able microencapsulated phase change slurry that is able to withstand shear stresses of the pump and other course surfaces of pipe and pipe joints. In this study, experiments were performed, to determine performance of microencapsulated phase change slurry over conventional heat transfer fluids. 
After certain pumping cycles, scanning electron microscopy has been done to analyze the conditions of shell material of polymeric capsule. Also, testing has been done to predict durability and life cycle of microencapsulated phase change slurry. 
TABLE OF CONTENTS

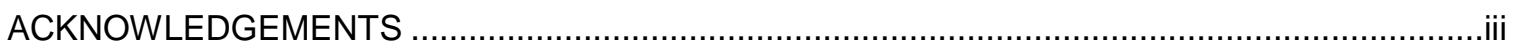

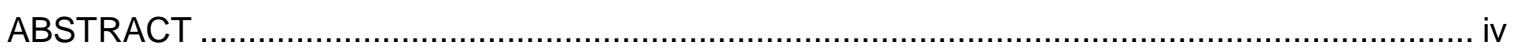

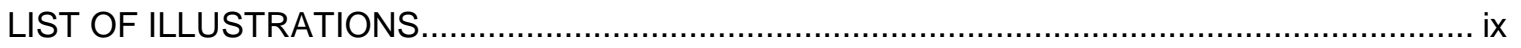

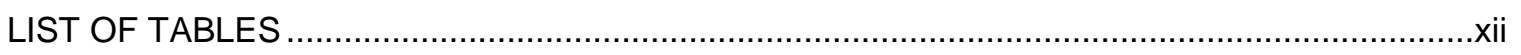

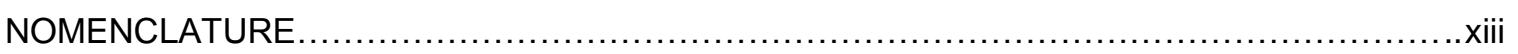

$\begin{array}{ll}\text { Chapter Page } & \text { Pas }\end{array}$

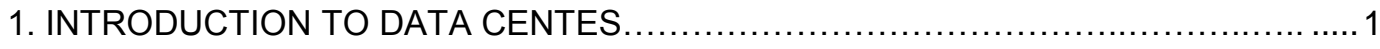

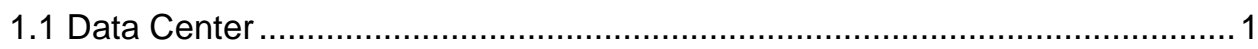

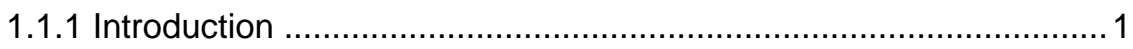

1.2 Modular Data Center Description ......................................................... 2

1.2.1 First Generation Data Center ................................................... 3

1.2.2 Second Generation Modular Data Center.................................... 4

1.2.3 Comparison between Traditional and Modular Data Center

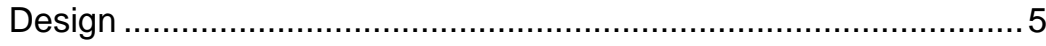

1.2.4 Power Usage Effectiveness: Metric for Energy Efficiency .............. 5

2. FREE COOLING AND PHASE CHANGE MATERIAL ............................................... 7

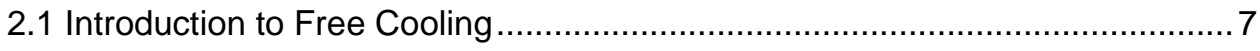

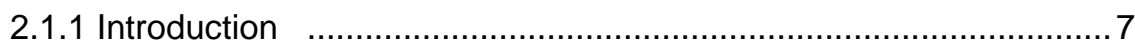

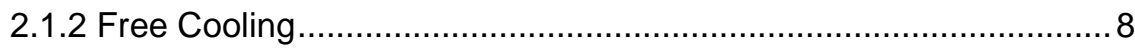

2.1.3 Types of Free Cooling Systems ............................................. 9

2.1.3.1 Air Side Economizers .............................................

2.1.4 Intel Proof of Concept .............................................................. 11

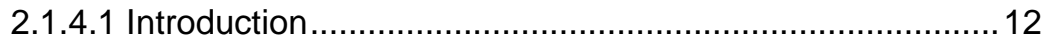


2.1.4.2 Results..................................................................... 12

2.2 Introduction to Phase Change Materials .................................................. 14

2.2.1 Energy density of different PCM per mass and per volume unit as a function of temperature................................................... 16

2.2.2 PCM Characteristics for Heat Transfer Fluid .............................. 16

2.2.3 Properties of Phase Change Materials ...................................... 17

2.2.4 Microencapsulation of Phase Change Materials.........................18

\section{DATA CENTER COOLING WITH MICROENCAPSULATED PHASE CHANGE}

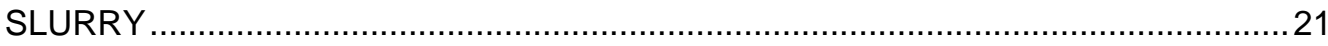

3.1 Introduction to Data Center Energy Consumption ......................................21

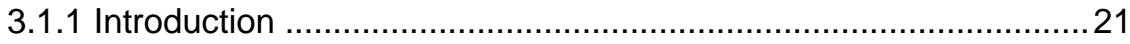

3.1.2 Objective and Approach ...................................................... 22

3.2 System Description and Configuration.................................................. 23

3.2.1 Description of Experimental Setup............................................23

3.2.1.1 Methodology of the Experiment ...................................24

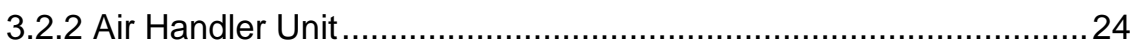

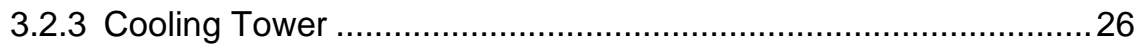

3.2.3.1 Advantages of the Cross Flow Design ............................26

3.2.3.2 Disadvantages of the Cross Flow Design .......................27

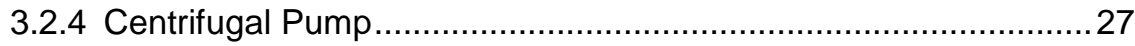

3.2.5 Microencapsulated Phase Change Slurry..................................28

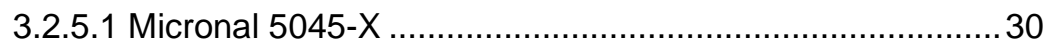


4. SCANNING ELECTRON MICROSCOPY FOR MICROENCAPSULATED PHASE

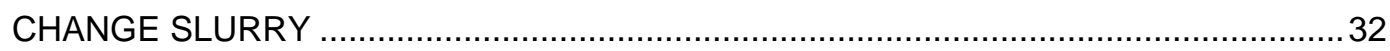

4.1 Scanning Electron Microcopy Working ................................................. 32

4.1.1 SEM Sample Preparation for mPCS …..................................... 33

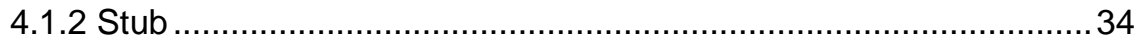

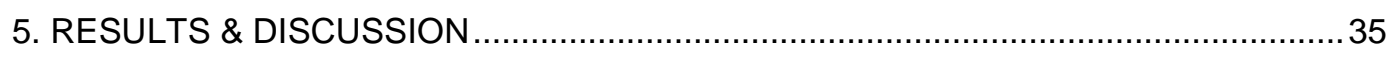

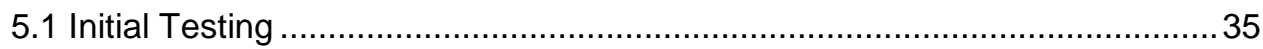

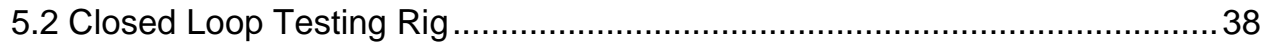

5.2.1 Experimental Results ....................................................... 40

5.3 Pump Compatibility Test setup for Micronal 5045-X.................................. 40

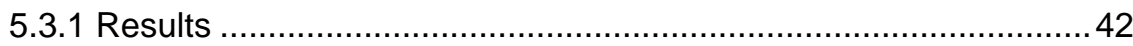

5.4 Testing for Water vs. mPCS as Thermal Storage Fluid .............................. 46

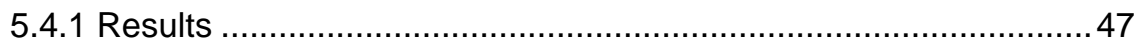

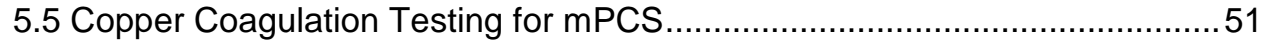

5.6 Final Testing with mPCS Slurry ....................................................... 51

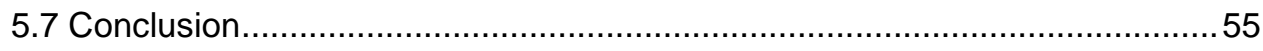

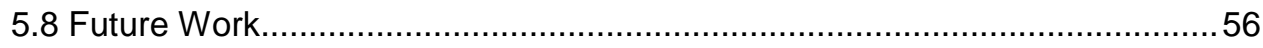

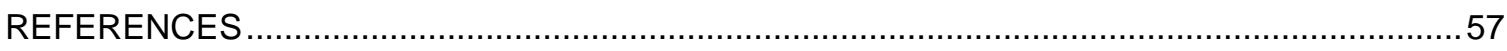

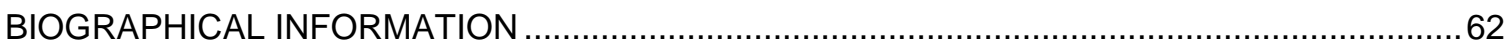




\section{LIST OF ILLUSTRATIONS}

Figure

Page

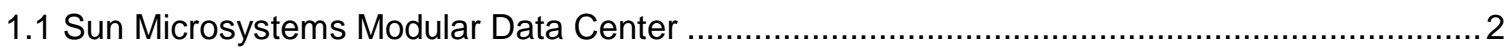

1.2 Raised Floor Air Cooling and Cold Aisle Containment System .......................................... 3

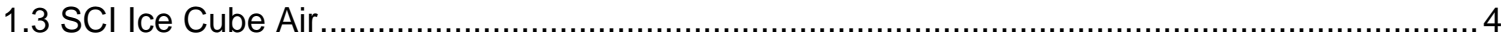

1.4 PUE Comparison for Different Cooling Designs .............................................................. 6

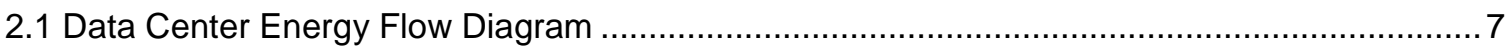

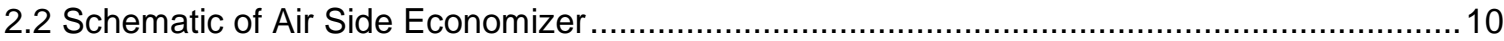

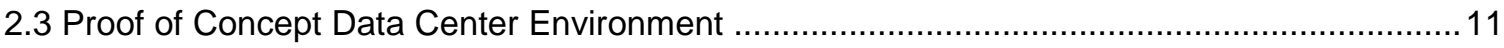

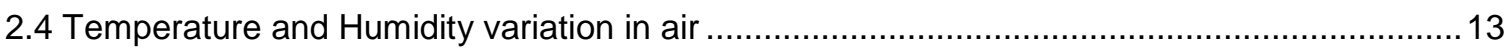

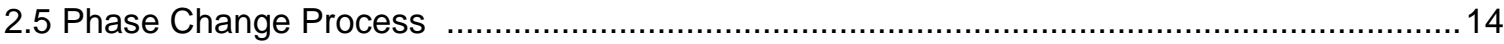

2.6 General classification of Phase Change Material ............................................................ 15

2.7 Specific enthalpy as a function of temperature for different materials, per mass unit [kJ/kg]

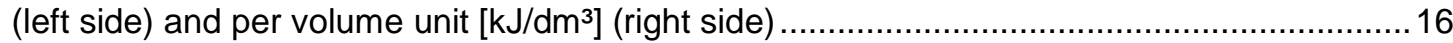

2.8 Microencapsulated Phase Change Material Process .........................................................19

3.1 Energy Consumption Distribution of Data Center ..............................................................22

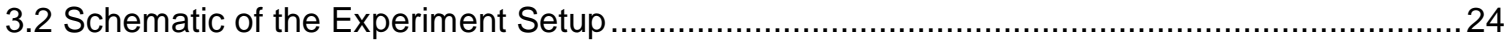

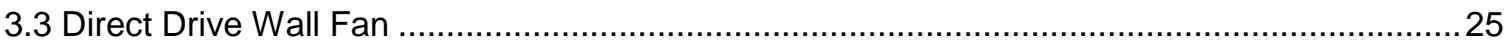

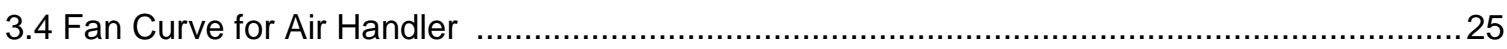

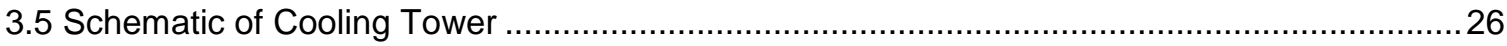

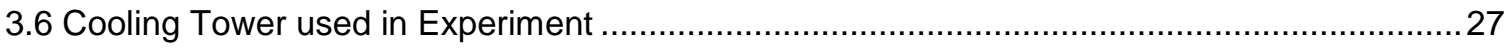

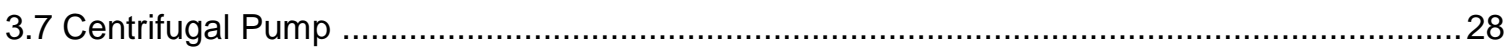

3.8 General Appearance of Phase Change Slurry and SEM Image .........................................29

3.9 Specification of the Micronal 5007-X

ix 


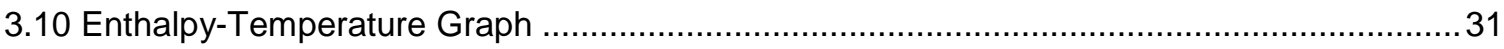

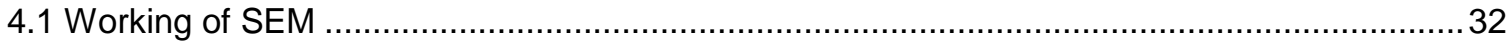

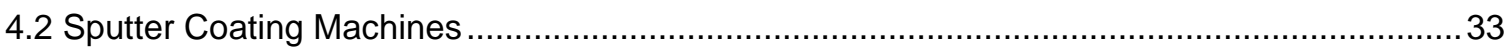

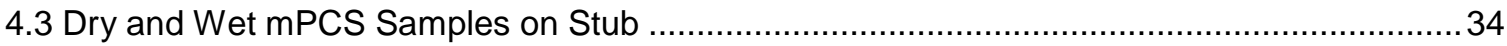

4.4 The general appearance of the powder mPCM capsule on the left and its SEM image on

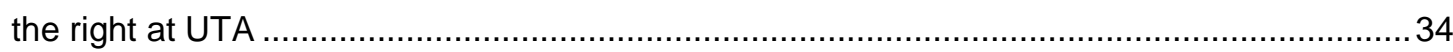

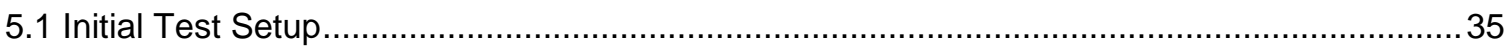

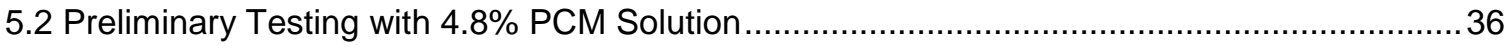

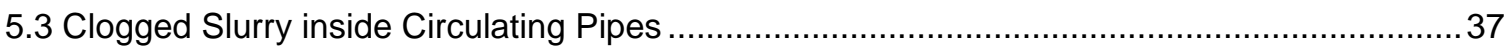

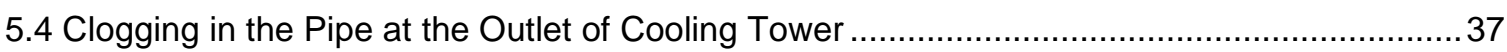

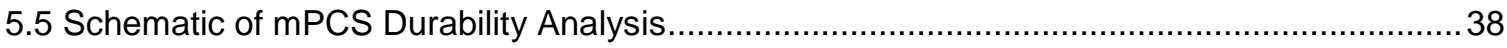

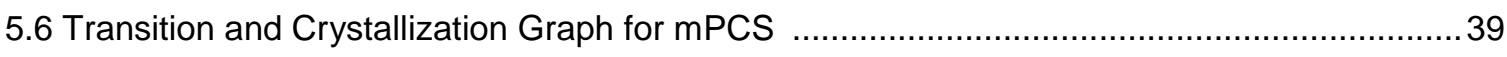

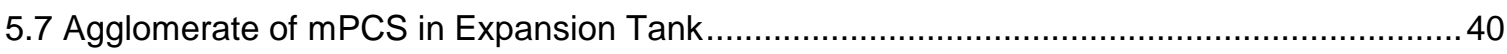

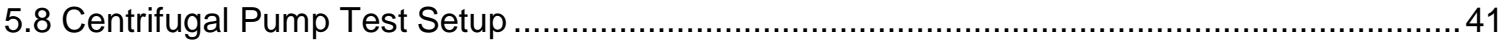

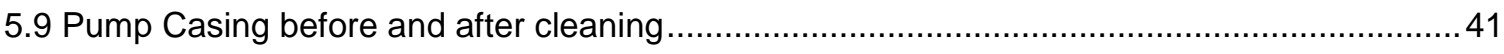

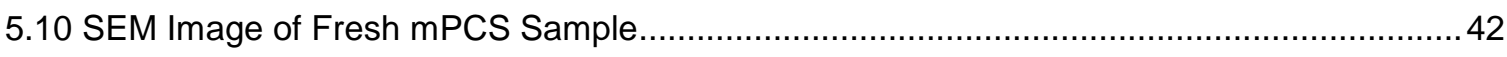

5.11 Measured Particle Diameter of Fresh mPCS Sample ....................................................... 42

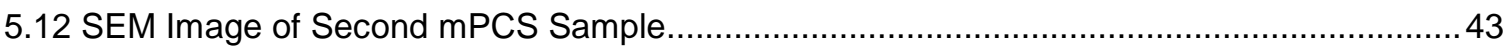

5.13 Measured Particle Diameter of Second mPCS Sample.................................................... 43

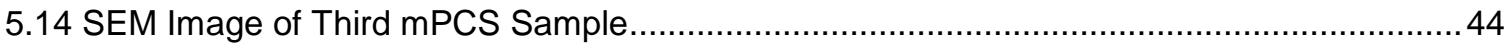

5.15 Measured Particle Diameter of Third mPCS Sample .......................................................... 45

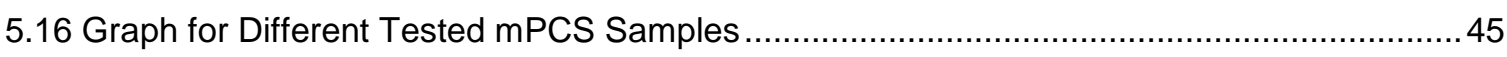

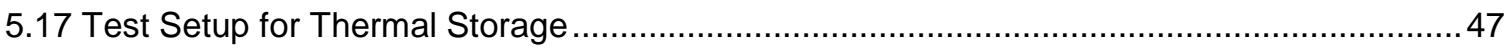

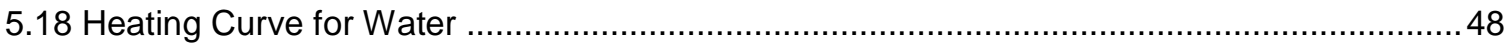




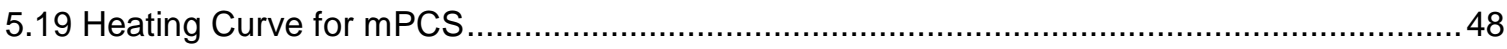

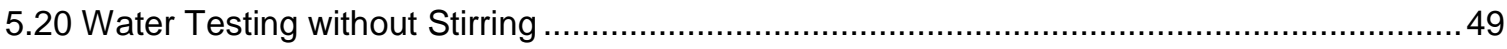

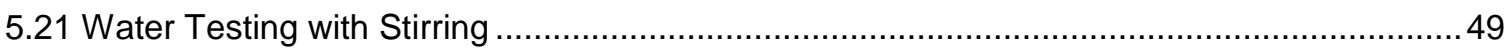

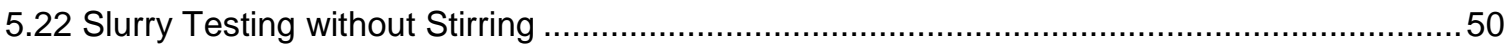

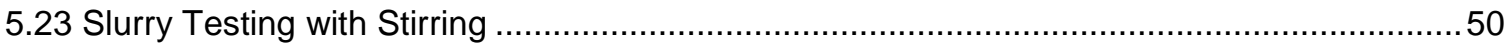

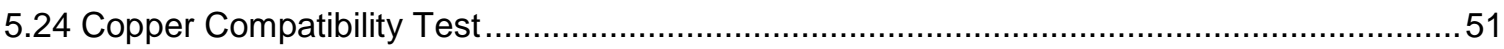

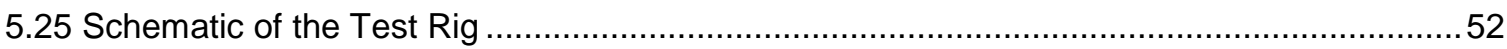

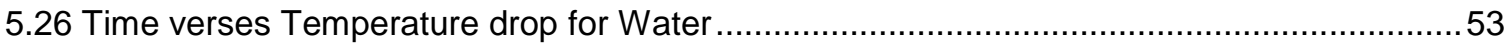

5.27 Temperature difference due to Heat from Pump …........................................................ 53

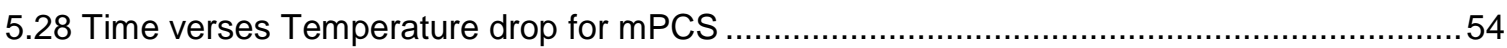

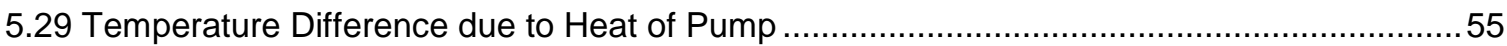




\section{LIST OF TABLES}

Table

Page

1.1 Comparison between Traditional and Modular Data Center Design ........................................ 5

2.1 Difference between Traditional and Free Air Cooling Technology ........................................ 8

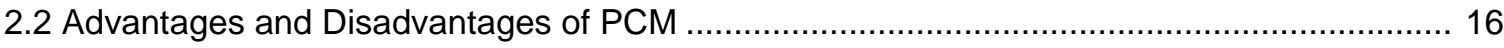

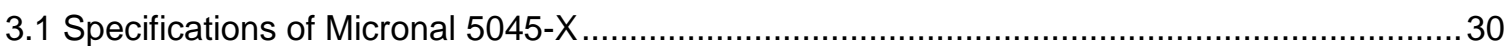

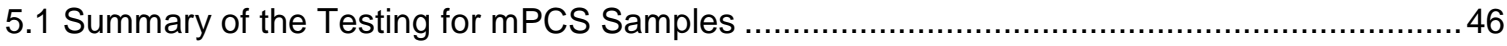

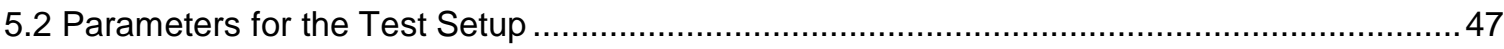




\section{NOMENCLATURE}

$C p=$ suspension's specific heat

$q_{w}=$ heat flux across the pipe wall

$R=$ radius of pipe

$k=$ suspension's thermal conductivity

$\mathrm{Cm}=$ mass fraction of $\mathrm{PCM}$ in suspension

$\lambda=$ PCM's latent heat of fusion 


\section{CHAPTER 1}

\section{INTRODUCTION TO DATA CENTERS}

\subsection{Data Center}

\subsubsection{Introduction}

Data center is a facility that contains various computer related equipment's most commonly known as IT equipment. IT equipment can be further sub divided as data processing servers, data storage servers and network equipment's. In addition data center also house uninterrupted power supplies (UPS), Computer room air conditioner (CRAC) units and power cooling modules [1].

With ever increasing in advancement in technology, the rise in demand for the important work of data centers has created a noticeable impact on the power grid. Moreover data center dominates in almost every field these days. In fact, data centers can be 40 times more energy intensive than a standard office building and require higher levels of power and cooling [2].

Traditional data centers were constructed more like buildings with; in such a way that they have very less natural ventilation the reason being they were designed to house IT equipment's. It is important that the operating environmental conditions of the data center falls under the recommended ASHRAE range for following criteria [1].

Further, traditional data centers design is almost obsolete now days. The reason being it is not flexible to alter the installed components, moreover or if expansion is required it has to be accounted in count in the beginning itself. Also, traditional data center facility is fixed. So, if any new installation of racks is required all the components have to be delivered at the facility [3].

In order to make this process more efficient and less time consuming, new containerized type data center were introduced by Sun Microsystems as project black box or 
sun modular data center. The standard 20 foot shipping container was turned into modular data center which can house up to 280 servers. The power supply and cooling unit were installed externally. The advantage of modular center is that it can directly deploy at the facility which saves the lag time as well as it requires less skill labor as compared to traditional data center. Since then, several companies including, Google, Hewlett Packard, CommScope, PDI, Microsoft Amazon etc. have developed containerized data centers featuring state of the art technologies [4]. Following is the figure shows the sun micro modular data center.

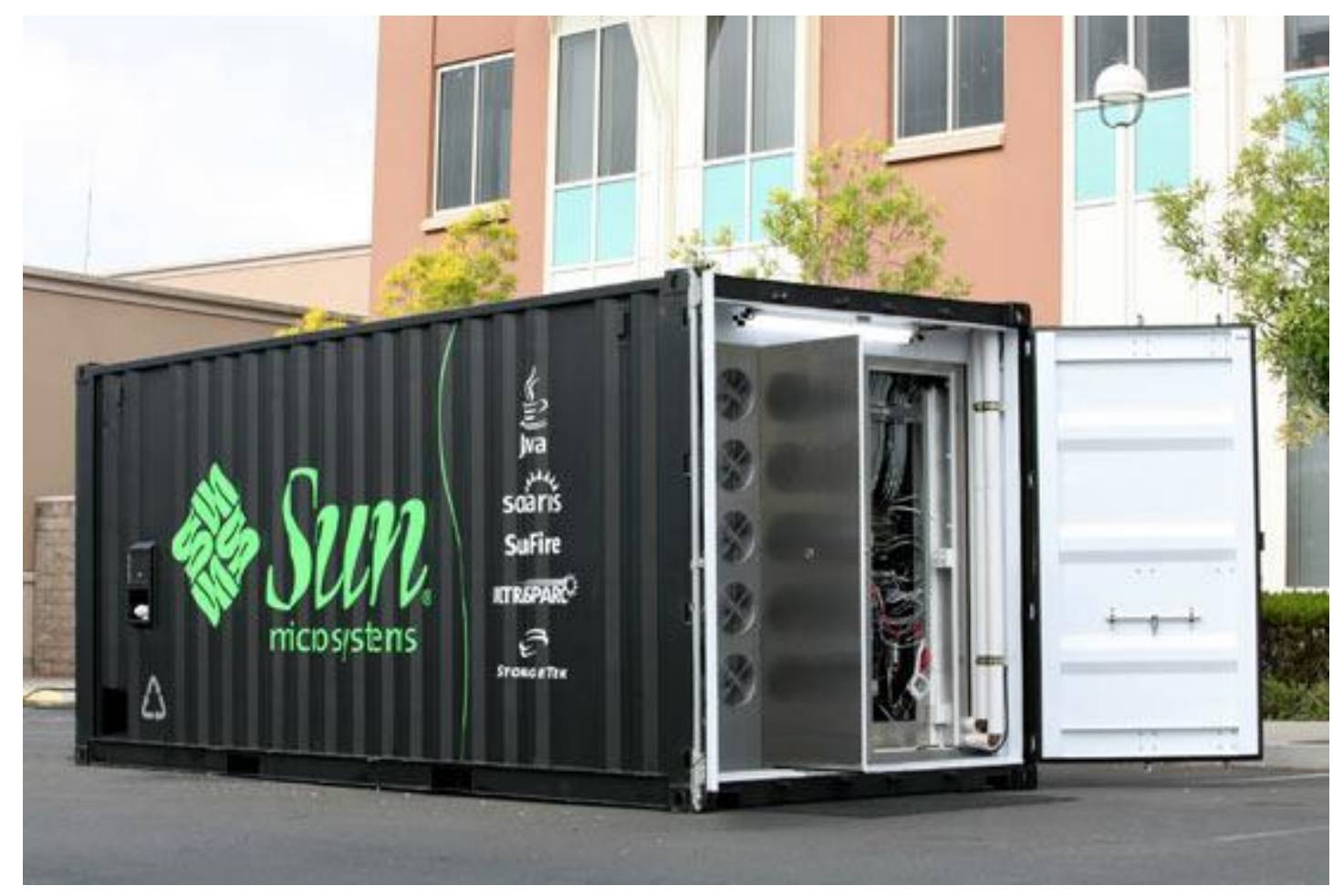

Figure 1.1: Sun Microsystems Modular Data Center [5]

\subsection{Modular Data Center Description}

Modular data centers are kind of more enhanced version of traditional data centers. It offers various advantages like ease of deployment and shipping, the outer frame is of fixed dimensions i.e. standard is followed which can be implemented anywhere. These overall results in lower operating costs compared to traditional data centers. Another importance of the modular data center is better thermal management and better air flow distribution. Also, free 
cooling technologies like air and water side economizers can be easily implemented in modular data center. Modular data center can be sub divided into two first generation and second generation $[3,4]$

\subsubsection{First Generation Data Center}

First generation modular data centers avails the standard CRAC units outside the container and they require some chilled water system or secondary refrigerant coils as cooling infrastructure support. In first generation data center many different configurations for rack placement and air cooling distribution can be accomplished. One of the most common configurations offered by first generation data center is hot aisle and cold aisle containment. Figure below shows the hot and cold aisle configuration [3, 4]

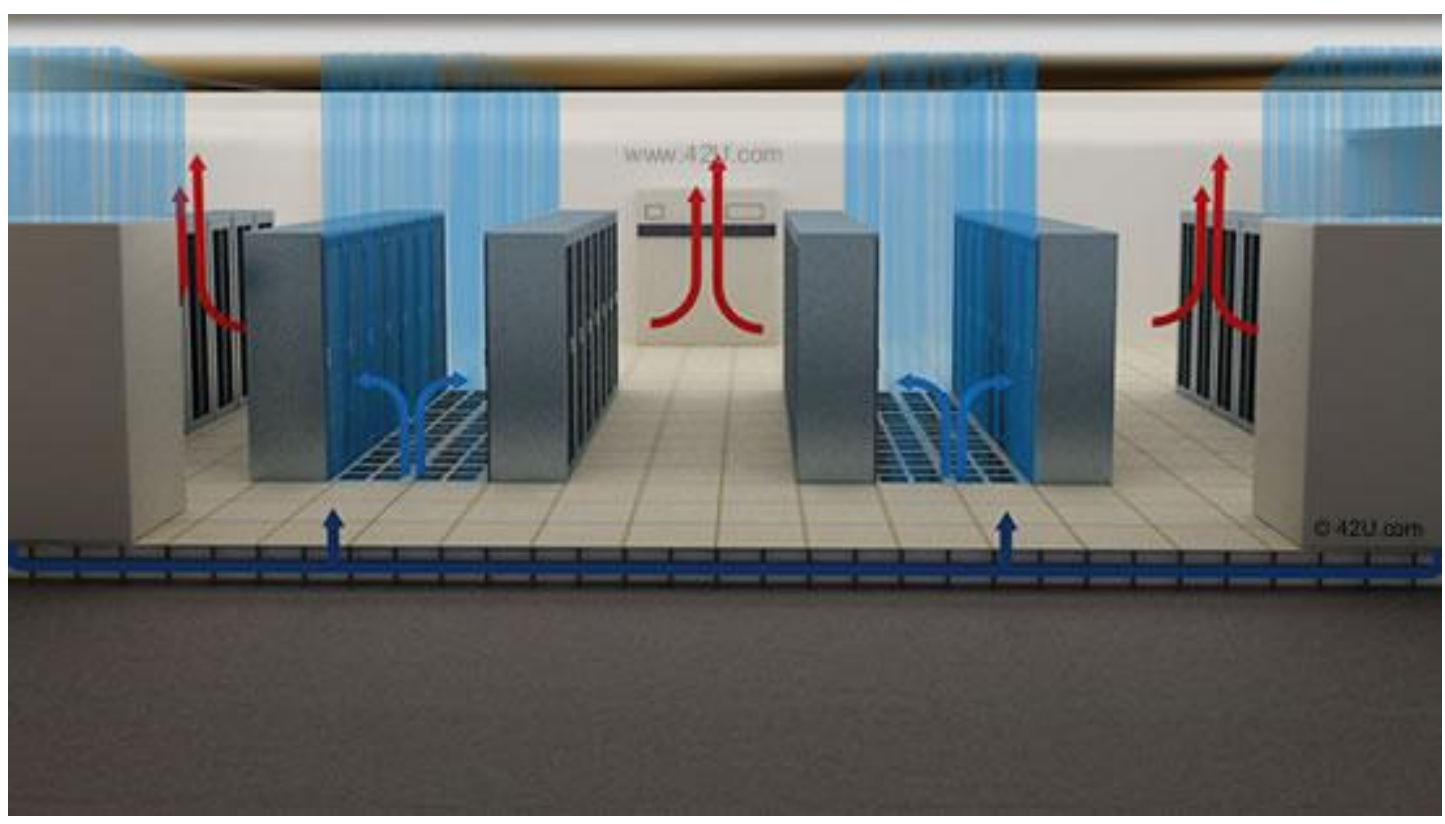

Figure 1.2: Raised Floor Air Cooling and Cold Aisle Containment System

The figure above describes the raised floor air cooling system: In this cool air from the power cooling module or CRAC units is supplied to the racks through under floor plenum. The blue curtains basically depict the cold aisle containment. Cool air from power cooling module is supplied to the racks through raised floor and is distributed to servers. Servers are housed in such a way that the front sides of servers which receive the cold air face each other. This 
configuration prevents mixing of hot and cold air and hence provides excellent hot/cold aisle containment. This configuration is employed in facilities where free cooling techniques doesn't work or may serve only as a short term solution for immediate expansion of existing facilities. First generation modular units are less energy efficient and more expensive than second generation units.

\subsubsection{Second Generation Modular Data Centers}

Second generation modular data centers directly make use of free cooling techniques sometimes along with any sustainable cooling source like evaporative cooling. This kind of data centers eliminate the need of chilled water supply or direct expansion cooling. These systems use free cooling, chilled water or direct expansion methods. Evaporative cooling, another highly efficient technique, is sometimes paired with free cooling when ambient air temperature reduction is needed. These systems may also include chilled water or direct expansion cooling units as backup when outside air temperatures dictate that use of economizers may not be favorable $[3,4]$

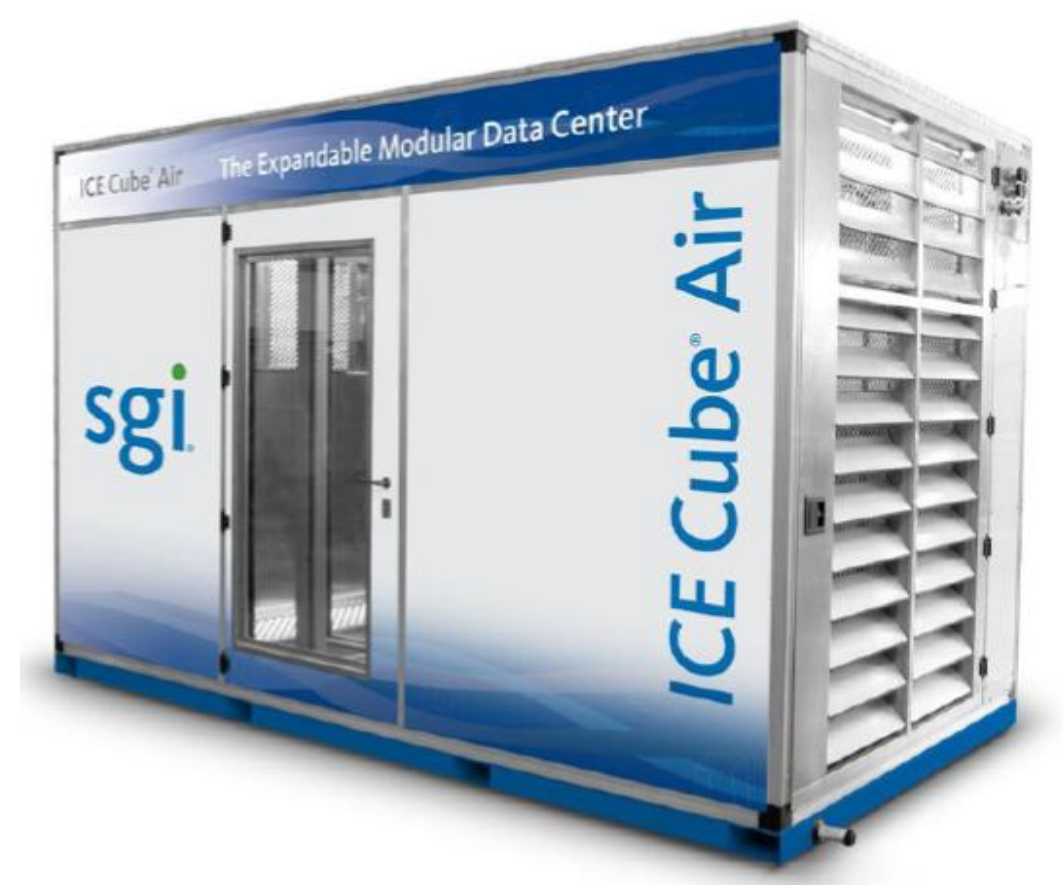

Figure 1.3: SCI Ice Cube Air [6] 
1.2.3 Comparison between Traditional and Modular Data Center Design

Table 1.1: Comparison of Primary Attributes $[3,4]$

\begin{tabular}{|c|c|c|c|}
\hline Primary Attributes & $\begin{array}{c}\text { Traditional "Brick and } \\
\text { Mortar" Data Center }\end{array}$ & $\begin{array}{c}\text { First Generation } \\
\text { Modular }\end{array}$ & $\begin{array}{c}\text { Second Generation } \\
\text { Modular }\end{array}$ \\
\hline Deployment & $\begin{array}{c}\text { Long - typically two } \\
\text { years from design to } \\
\text { commissioning } \\
\text { perhaps in months } \\
\text { depending on site } \\
\text { conditions and } \\
\text { available } \\
\text { infrastructure }\end{array}$ & $\begin{array}{c}\text { Same as First Gen. } \\
\text { Modular with } \\
\text { advantage that } \\
\text { reduced cooling } \\
\text { infrastructure is } \\
\text { required }\end{array}$ \\
\hline Capital Cost & $\begin{array}{c}\text { Highest - generally } \\
\text { thought to range from } \\
10-\$ 20 \text { million per } \\
\text { MW of IT capacity }\end{array}$ & $\begin{array}{c}\text { Lower - though there } \\
\text { is a lack of } \\
\text { documented } \\
\text { deployment costs }\end{array}$ & $\begin{array}{c}\text { Lowest - marginal } \\
\text { increase in cost of } \\
\text { unit, made up for } \\
\text { by reduced } \\
\text { infrastructure costs }\end{array}$ \\
\hline Operating Cost & $\begin{array}{c}\text { Variable, with legacy } \\
\text { data centers having } \\
\text { PUE's exceeding 2.0 } \\
\text { and best-in-class } \\
\text { designs approaching } \\
1.2 \text { or lower if using } \\
\text { outside air for cooling }\end{array}$ & $\begin{array}{c}\text { Similar to traditional } \\
\text { data center using the } \\
\text { same cooling type. } \\
\text { Pre-engineering and } \\
\text { better system } \\
\text { integration may } \\
\text { provide some } \\
\text { advantages. }\end{array}$ & $\begin{array}{c}\text { Similar to best in } \\
\text { class legacy data } \\
\text { centers that use } \\
\text { air-side cooling. }\end{array}$ \\
\hline
\end{tabular}

1.2.4 Power Usage Effectiveness: Metric for Energy Efficiency

Power usage effectiveness (PUE) is a standard/metric developed by The Green Grid consortium in order to determine energy efficiency within a data center [3, 4 \& 7]. Power usage effectiveness (PUE) is defined as:

$$
\text { PUE }=\text { Total Facility Power/IT Equipment Power }
$$

The ratio of the total power used by a data center facility to the IT equipment power supplied.

Total facility power includes all the components such as power generators, UPS systems and batteries and cooling systems such as chillers, cooling towers, pumps and CRAC units [8 Niket]. The IT equipment power includes components like, computing and storage servers, KVM switches and motherboards or any other electronic accessories used for monitoring and controlling the data center. 


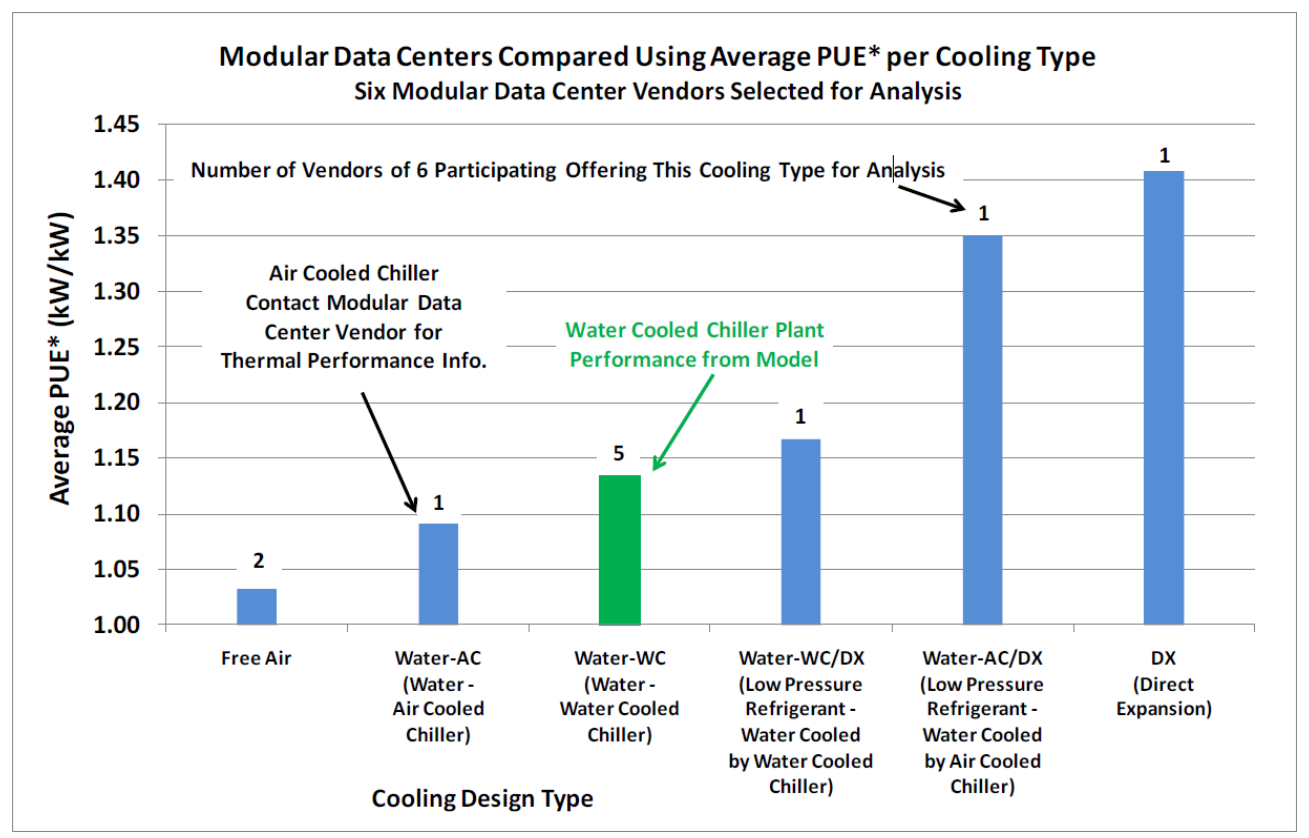

Figure 1.4: PUE comparison for Different Cooling Designs [3, 4 \& 8]

The ideal PUE value of 1.0 indicates 100 percent efficiency. This generalize that all the power that is being supplied is used up in the IT power only. The value of PUE generally ranges between 2.0 to 3.0 for data centers [3, $4 \& 9]$. 


\section{CHAPTER 2}

\section{FREE COOLING AND PHASE CHANGE MATERIAL}

\subsection{Introduction to Free Cooling}

\subsubsection{Introduction}

Data center accompanies large number of servers. The data center server's stores and process enormous amount of information in day to day services. In fact with the increase in information technology load trend to operate these servers for longer and continuous durations it consumes huge amount of energy. Despite these devices are becoming efficient day to day but simultaneously the power required to run these devices is also increasing. Continuous operation of these devices leads to the generation of a large amount of heat. One of the major goals to improve the efficiency of data center is to bring down the value of power usage effectiveness. The average power usage effectiveness value ranges between 2 and 2.5. The major portion of the energy consumption from these servers contributes to the cooling system. The cooling system needs tremendous amount of energy to lower down the temperature of the servers. This further increase in energy has caused an increase in electricity consumption for cooling systems. Figure 2.1 show energy consumption for different systems within a data center

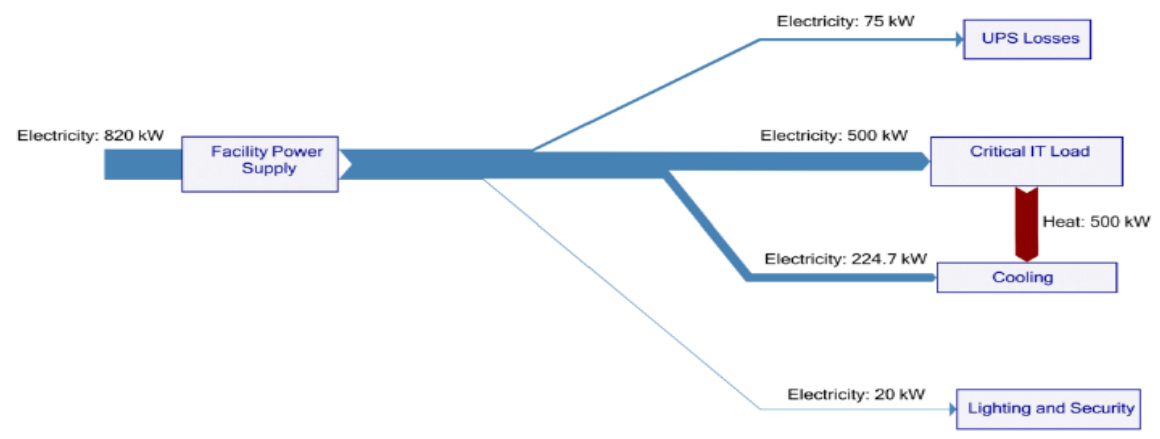

Figure 2.1: Data Center Energy Flow Diagram [10] 
In order to reduce the consumption for these cooling systems or in other way to make these cooling systems more efficient, free cooling technique is widely being adopted. Free cooling is simple technology that takes advantage of external ambient conditions in order to cool servers $[3,4]$

\subsubsection{Free Cooling}

Free cooling uses ambient conditions for cooling information technology (IT) equipment completely or part of the time (coupled with traditional computer room air conditioning (CRAC) units). This system requires less energy since it doesn't use compressors for cooling incoming air. Free cooling is the most efficient cooling alternative for data center cooling. Mostly under free cooling ambient air is introduced into the data center through filters, and is then forced by fans that provide the required flow rate to directly cool the server equipment. The heated server exhaust air is then vented back out to the ambient. This cooling method is highly effective at reducing energy consumption for data centers in cooler climates where it can be utilized for a significant portion of the year [11]. The basic difference between conventional and free cooling technology where applicable is shown is Table 2.1 .

Table 2.1: Difference between Traditional and Free Air Cooling Technology

\begin{tabular}{|l|l|}
\hline \multicolumn{1}{|c|}{ Traditional Cooling Technology } & \multicolumn{1}{c|}{ Free Air Cooling Technology } \\
\hline $\begin{array}{l}\text { Use vapor compression systems or chilled } \\
\text { water system to cool the servers. }\end{array}$ & Make use external ambient conditions \\
\hline $\begin{array}{l}\text { These cooling systems require large and } \\
\text { heavy mechanical systems such as } \\
\text { compressors and condensers for rejecting } \\
\text { heat to the atmosphere. }\end{array}$ & $\begin{array}{l}\text { Uses outside air completely or part of time } \\
\text { with traditional units, thus significantly } \\
\text { reducing the use of mechanical systems. }\end{array}$ \\
\hline $\begin{array}{l}\text { Requires cooling tower to bring down the } \\
\text { temperature of the primary refrigerant }\end{array}$ & $\begin{array}{l}\text { The hot exhaust air can be released to the } \\
\text { atmosphere directly. }\end{array}$ \\
\hline $\begin{array}{l}\text { This entire process consumes a large } \\
\text { amount of energy. }\end{array}$ & $\begin{array}{l}\text { This pose a very good solution for cutting } \\
\text { down energy costs. }\end{array}$ \\
\hline
\end{tabular}




\subsubsection{Types of Free Cooling Systems}

Free cooling can be further specified into two: air side economizer and water side economizer. In air side cooling, cold air is pulled in through filters from outside and circulated through the servers and other dissipating units at the entrance. The heat is carried away and is finally rejected to the atmosphere. However, air side cooling is less expensive and reduces the energy costs but large volume of air is required to cool data center. Large fans are required to pull in an enormous quantity of air through the filters. The main problem encounters with the filters is that they have to be replaced periodically. Filters are used to prevent the entry of any contaminants such as dust, moisture etc. in the data center. With the passage of time the filters becomes dirty and offers very high pressure drop. Also, it requires larger ducts for air and offers high pressure drop. Although this cooling technique sounds very simple, it may not be practical to apply for cooling large volumes of area within a data center as pulling in so much air and filtering it is extremely difficult $[3,4]$

Water side economizer accompanies use heat exchangers at the back of the server cabinets known as rear- door heat exchangers. In this technology water is circulated through the channels in rear door heat exchangers. This is much more efficient in transferring heat than air per unit volume. Finally heat from the water is rejecting to the atmosphere using cool ambient air with the help of cooling towers. Moreover, the water side cooling can be achieved by integrating it with centralized chilled water systems and condensers $[3,4]$

Our main interest in this project is to see the effectiveness of air side economizer with closed loop system circulating phase change slurry. The water most commonly used is replaced with the phase change slurry in the system $[3,4]$

\subsubsection{Air Side Economizers}

Air side economizers as described above brings in outside air and made it through a control mechanism which to regulate it based upon its conditions. Air side economizers utilize a system of dampers to control and sensors. The dampers are used to vary the amount of the 
flow rate of air required. The Sensors are employed for monitoring outside and inside air temperature and humidity. If external conditions suit the recommended temperature and humidity range it is allowed to pass through filters and then directly into the server cabinets.

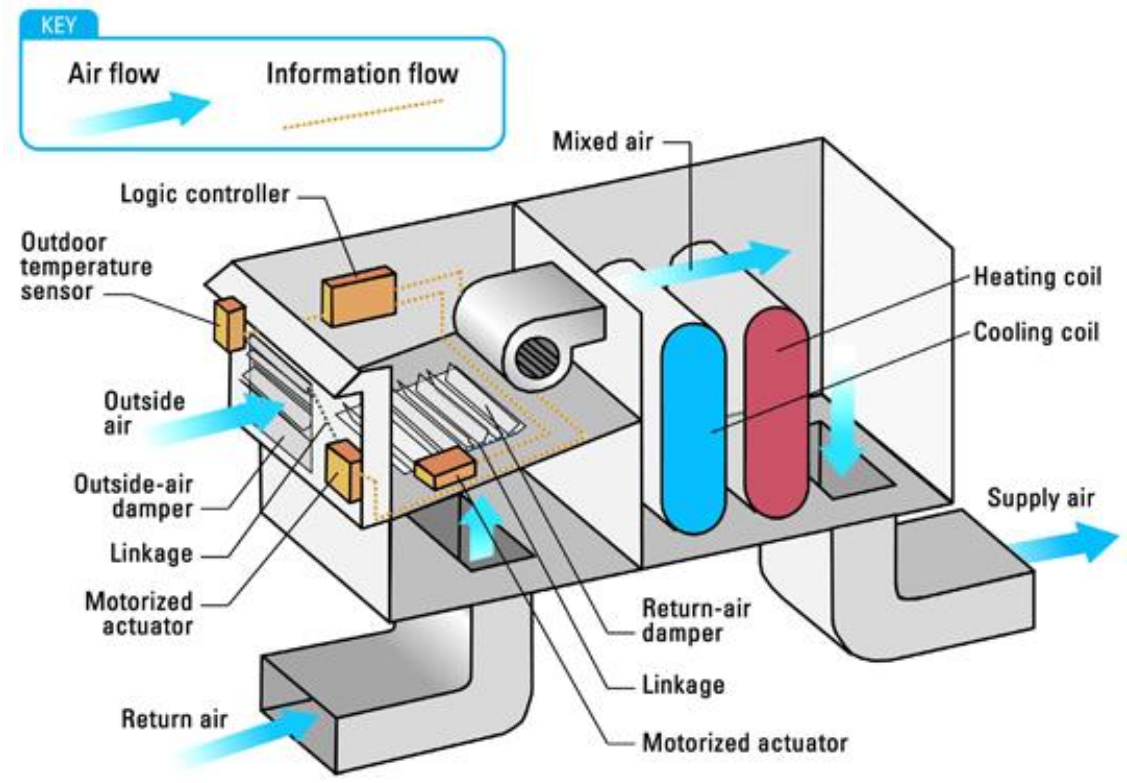

Figure 2.2: Schematic of Air Side Economizer [12]

While passing through the filters, all the major contaminants are filtered out. The filtered air is circulated across the portion that another cooling and heating unit. At times the heating or cooling have to be used to bring the outside air temperature and humidity into the recommended envelope before it enters the data center. Also, that portion has capability to mix hot data center exhaust air with the filtered incoming air. In case large volumes of outside air are introduced into the system, exhaust dampers maintain the pressure by driving out unnecessary air. Hence, use of air side economizers helps in reducing the use of air conditioning units and chilled water systems significantly.

A study by Shehabi et al. [13] compares the energy consumption rates of traditional data centers with air side economizers and water side economizers in five different climate zones in the state of California. The report suggested the use of airside economizer have resulted in better performance in all climate zones. In addition another study by Syska 
Hennessy Group [14] suggested that for the region of San Francisco, outside air can be used for almost throughout the year.

Saket et al. [15] studied the effect of air side economizers for various cases using numerical models. The models were designed and analyzed to determine their performance at different operating conditions. Four different cases were analyzed: The first case includes conventional data center with CRAC units and an under floor plenum supply. The other three configurations contain air side economizers. The system parameters such as heat load, flow rate, rack layout and percentage open area ratio for the tiles remained the same including system dimensions. The use of air side economizers promised substantial energy savings in comparison with conventional data center with CRAC units.

\subsubsection{Intel Proof of Concept}

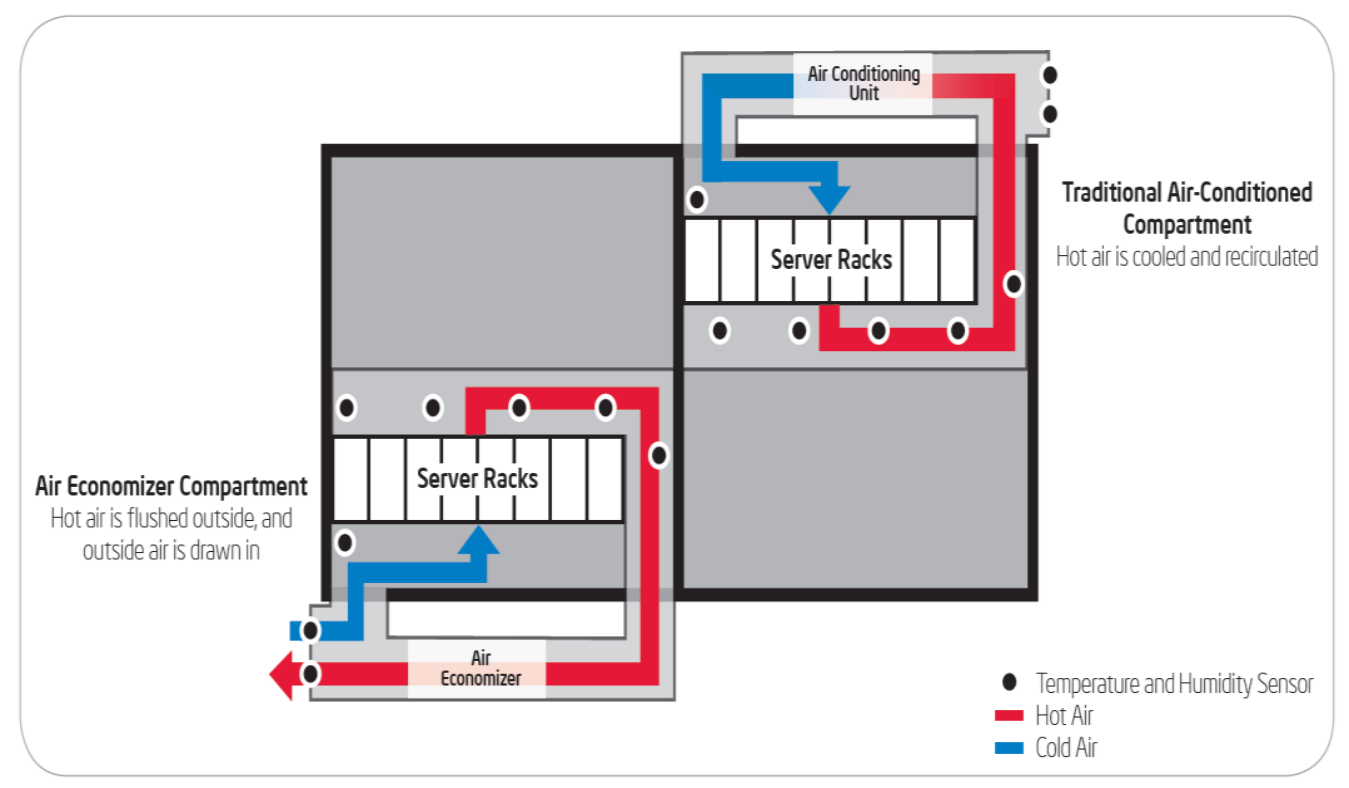

Figure 2.3: Proof of Concept Data Center Environment [16] 


\subsubsection{Introduction}

Intel conducted a large proof of concept test using approximately 900 production design servers at a data center. The region selected for this test has a temperate desert climate with relatively low humidity. The test was conducted for over the period of 10 months (October 2007August 2008)

The test setup includes trailer with dimensions of 1000-sqaure-foot (SF). The trailer was divided exactly into two halves with 500 SF. To downsize the cost low grade direct expansion DX units were installed in one compartment. The other compartment was modified to operate completely on economizer mode, drawing $100 \%$ fresh air from the outside. Temperature and humidity sensors were installed to monitor the conditions in each compartment.

The economizer mode was set to maintain the air temperature ranging from $65^{\circ} \mathrm{F}$ to $90^{\circ} \mathrm{F}$. This fulfills the one of the goal to test the acceptable limits of operating temperature. Chillers were installed to cool the supply air temperature if it exceeds $90^{\circ} \mathrm{F}$. Also, if the temperature of the supply air is below the $65^{\circ} \mathrm{F}$ the hot exhaust air was mixed with the supply air to meet the required temperature. No attempt was made to control the humidity. In addition, minimal filtering was applied to the supply air using standard MERV rating household filters. This filter permits the fine dust to pass through [16].

\subsubsection{Results}

During the testing period the economizer mode was subjected to varied temperature and humidity range. The temperature varied from $65^{\circ} \mathrm{F}$ to $92^{\circ} \mathrm{F}$ and humidity value varied from $4 \%$ to $90 \%$ [16]. The Fig 2.4 indicates the variation. 


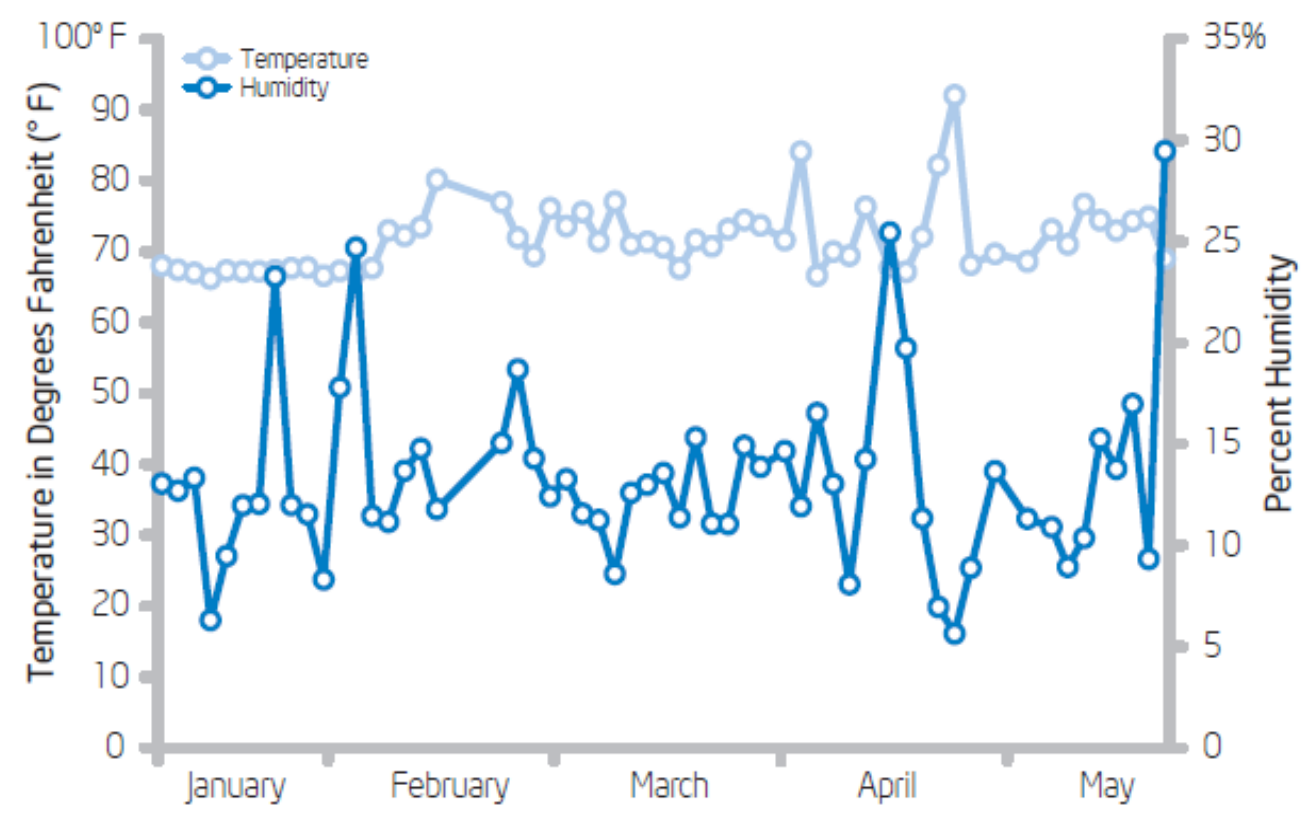

Figure 2.4: Temperature and Humidity variation in air [16]

When both the compartments of the trailer were cooled with DX units the total power consumption was around $500 \mathrm{KW}$. When one of the compartments of the trailer was replaced by the economizer mode, it resulted in the net savings of $111.78 \mathrm{KW}$ to $28.6 \mathrm{KW}$.

At the end, the servers and interior of the compartment were fully covered with the layer of dust. Although there is huge variation in humidity and temperature, there was a minimal difference between the server failure rates. The failure rates were $4.6 \%$ in economizer mode and 3.86 in direct expansion unit.

The Air side economizer has limitation based upon the region in which they lie. In other words they can be very suited in the area where the temperature remain in the ASHRAE recommended range for data centers throughout year. In order to overcome this problem sometimes air-side economizers are used along with other cooling technologies to bring down the temperature in the recommended envelope. One of the less expensive technology includes, evaporative cooling both direct and indirect. Evaporative cooling promises huge energy savings 
with respect to direct expansion units. Evaporative cooling uses water as a primary coolant to lower the temperature of the supply air.

In order to increase the heat capacity of water microencapsulated phase change material is dispersed in it. The microencapsulated phase change material slurry is mixture of microencapsulated phase change material dispersed in carrier fluid, e.g. water or oil. The mPCM slurry promise higher heat transfer capacity than other conventional heat transfer fluids.

\subsection{Introduction to Phase Change Materials}

Phase change material possesses quality to store and release large amount of thermal energy while changing its phase, for example: from solid-liquid, liquid to vapor. Best example to describe phase change material is water and ice. When ice is heated, during its phase change from solid to liquid its temperature remains constant. The temperature does not change till two phases (solid - liquid) exist simultaneously. In addition the inflowing energy is used up in the phase transition.

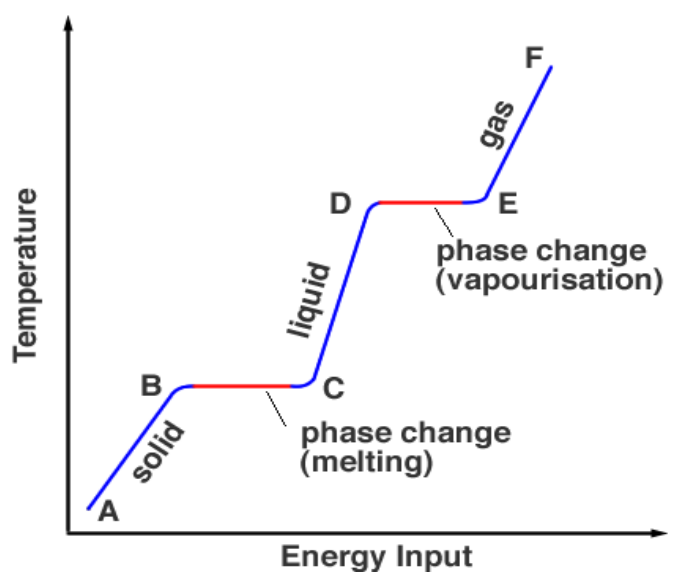

Figure 2.5: Phase Change Process [17]

The energy consumption in phase change is known as latest heat of fusion or melting. In contrast, water takes up or rejects heat sensibly. It takes 333 kilojoules of energy to melt one kilogram of ice at $0^{\circ} \mathrm{C}\left(32^{\circ} \mathrm{F}\right)$ to produce one kilogram of water. However, the same amount of energy would also be able to heat a kilogram of water from $0 \mathrm{C}$ to approx. $80^{\circ} \mathrm{C}\left(176^{\circ} \mathrm{F}\right)$. Phase change material acts as thermal storage and they release and absorb heat at constant 
temperature. In addition, these materials suits very well for the thermal management as they can absorb nearly 5-14 times more thermal energy per unit their volume. Moreover, the flexibility of these materials to lie under varied heat of fusion makes it more compatible.

Further PCM can be mainly classified into three categories namely organic, inorganic and eutectic [18]. Following figure represents the PCM classification by sharma et al [19]

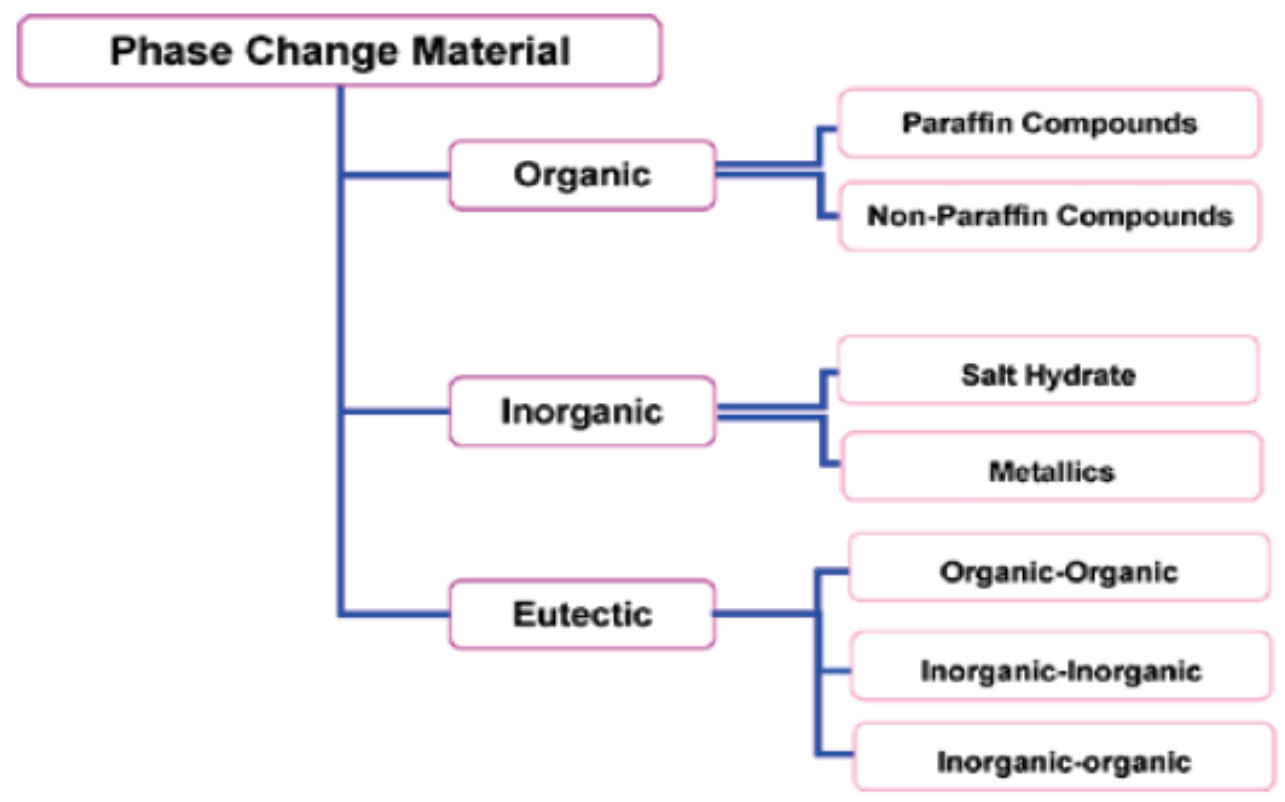

Figure 2.6: General classification of Phase Change Material [19]

Generally there are two main groups of PCMs: Organic (paraffin compounds) and Inorganic (salt hydrates). Paraffin promises more stability in terms of thermal cycling, i.e. it can undergo large number of phase changes maintaining the same characteristics. Despite, being more stable in comparison to salt hydrates they are flammable and possess lower melting enthalpy and density. The salt hydrates are corrosive in nature and their implementation needs careful examination. Also, salt hydrates undergo sub-cooling thus inhibiting the release of stored energy [20]. 
Table 2.2: Advantages and Disadvantages of PCM [20, 21]

\begin{tabular}{|c|c|}
\hline \multicolumn{1}{|c|}{ Organic (Paraffin) } & In Organic(Salt Hydrate) \\
\hline Advantages & - high melting enthalpy \\
- not corrosive & high density \\
- No or little sub cooling & \\
\hline Disadvantages & $\begin{array}{l}\text { Disadvantages } \\
\text { - lower melting enthalpy } \\
\text { - lower density } \\
\text { - flammable }\end{array}$ \\
\hline
\end{tabular}

2.2.1 Energy density of different PCM per mass and per volume unit as a function of temperature determined by T-History method (Marin et al., 2002)
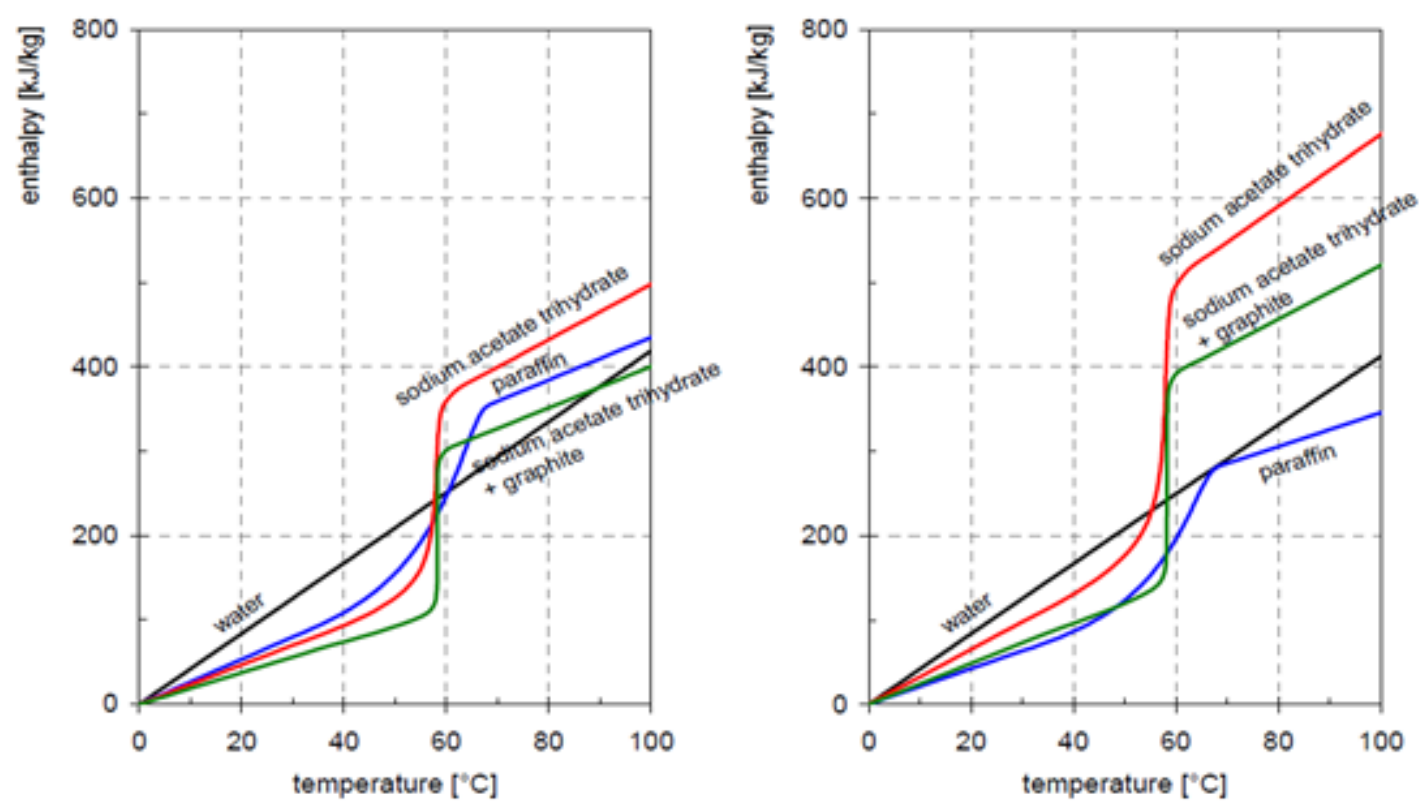

Figure 2.7: specific enthalpy as a function of temperature for different materials, per mass unit [kJ/kg] (left side) and per volume unit [kJ/dm³] (right side) [20]

\subsubsection{PCM Characteristics for Heat Transfer Fluid [20]}

In general PCM should meet the standard criteria to be used as heat transfer fluid:

- Suitable melting temperature

- High melting enthalpy per volume unit $\left[\mathrm{kJ} / \mathrm{m}^{3}\right]$ 
- High specific heat $[\mathrm{kJ} /(\mathrm{kg} \mathrm{K})]$

- Low volume change due to the phase change

- High thermal conductivity

- Cycling stability

- Non-flammable,

- Non-poisonous

- Non-corrosive

\subsubsection{Properties of Phase Change Materials}

Phase Change Material performance in heat exchange systems depends on factors such as the Stefan number, mass fraction, and the latent heat of fusion. In order for the PCM to be at optimal effectiveness, the Stefan number must be lower than 1 and defined as follows:

Where:

$$
\begin{aligned}
& \qquad S t=\frac{C_{p}\left(q_{w} \frac{\mathrm{R}}{\mathrm{K}}\right)}{C_{m}} \\
& C p=\text { suspension's specific heat } \\
& q w=\text { heat flux across the pipe wall } \\
& R=\text { radius of pipe } \\
& k=\text { suspension's thermal conductivity } \\
& C m=\text { mass fraction of PCM in suspension } \\
& \lambda=\text { PCM's latent heat of fusion }
\end{aligned}
$$

High mass fractions and latent heat of fusion would intuitively be preferred because they permit a higher heat capacity. However, high mass fractions increase slurry viscosity, which in turn demands more pumping power [22] 


\subsubsection{Microencapsulation of Phase Change Materials}

PCM possess high thermal storage capacity but on the other hand they have relatively low thermal conductivity. In general there are two ways by which we can enhance the thermal conductivity of PCM:

- The heat transfer distance between PCM i.e. by conduction can be reduced: this can be achieved by microencapsulating the material into small capsules.

- Also, thermal conductivity of PCM can be increased by embedding structures of materials with high conductivity. This is done by adding graphite powders into the PCM which increases its thermal conductivity by the factor of 10-20 (Öttinger, 2004), but also creates a kind of carrier structure that inhibits the segregation of salt hydrates and therefore improves their cycling stability segregation of salt hydrates and therefore improves their cycling stability. [20]

The concept of a carrier fluid with encapsulated particles of phase change materials was first introduced by Mehalick and Tweedie [18, 23]. Micro-encapsulation is a process in which small particles of PCMs are coated by some shell material and are produced in the form of small capsules. These capsules maintain uniform wall surface and its size ranges from few micrometers to few millimeters. The material inside the shell material is referred as core material. In addition, by microencapsulating the core material remains isolated from the entire system, it changes its phase inside the shell material thus preventing it from any kind of fouling or contamination problem [22] 


\section{Micro-capsule \\ Wall material}

\section{Core material (PCH)}

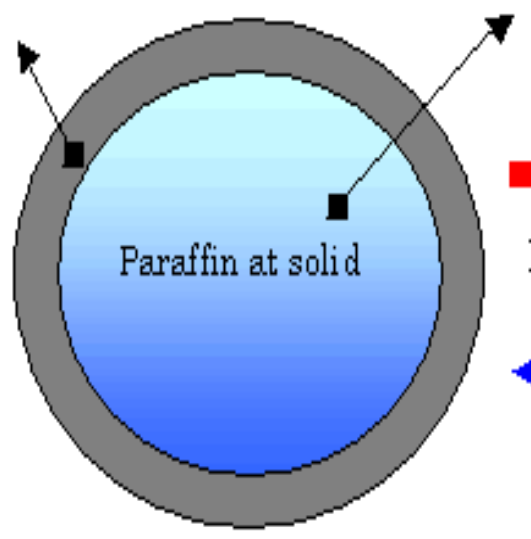

\section{Cool thermal accumulation}

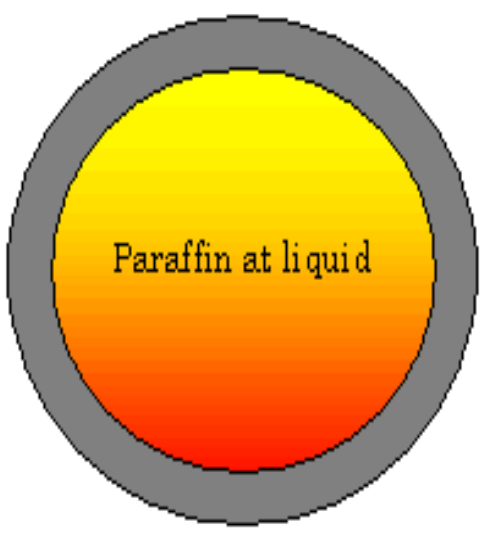

Warmil thermal accumulation

Figure 2.8: Microencapsulated Phase Change Material Process [24]

The shell material is generally a polymer and the particles can be either multi phase or liquid solid \& gas. The two most commonly used microencapsulation techniques include, in-situ polymerisation and interfacial polycondensation. In order to obtain stable microcapsules In-situ polymerization technique is often employed. The procedure for In-situ polymerization is as follows: at beginning the shell material is dissolved into water and/or the paraffin; then by using high shear mixer the paraffin is added into the mixture and emulsified to yield the designed size distribution. The polymerization of the paraffin microcapsules is started by changing the $\mathrm{PH}$ value and temperature, or by adding flocculation or coacervation agents. At last the obtained microcapsules are filtered, washed and dried. It is possible to obtain homogeneous slurry if the shell materials with hydrophilic groups like melamine resin are used. Su et al. [25] reported that the shells of microencapsulated phase change materials do not have big impacts on the phase change temperature of pure paraffin.

This microencapsulation offers various features such as high energy storage density, low pumping power requirements, and high heat transfer rates. There are various phase- 
change materials, e.g. paraffin, fibers, foams and composites which are developed to enhance thermal efficiency as coolants, when used in conjunction with liquids [26, 27 and 28]

In comparison to liquid cooling techniques, for example: using water as a coolant, it has been seen that microencapsulated phase change slurry perform higher heat transfer rate. Also, microencapsulated slurry founds its application in many fields some of them includes: in electronic cooling using micro channels, Various HVAC systems. Studies by Wang et al [29] depicts that PCM slurry offer better heat transfer coefficient in laminar fluids than single phase fluids but depicts complex phenomena at low turbulent conditions. Moreover, Microencapsulated Phase Change Material Slurry (MPCS) flowing in a circular tube were experimentally and numerically investigated, which showed enhanced convective heat transfer by Zeng et al [30] Numerical analysis of convective heat transfer enhancement with microencapsulated phase change material slurries have also been investigated by Hu and Zang [31]. The forced convection heat transfer with microencapsulated phase change material slurries has also been theoretically investigated by Charunyakorn et. al [32], Roy and Avanic [33].

Despite, its high thermal storage and high heat transfer coefficients; the major limitations with phase change material is its tendency to clog in distribution pipes and lower heat transfer rates in heat exchangers. A literature review suggests that microencapsulating the PCM can eliminate this problem while maintaining its original properties [22]. 


\section{CHAPTER 3}

\section{DATA CENTER COOLING WITH MICROENCAPSULATED PHASE CHANGE SLURRY}

\subsection{Introduction to Data Center Energy Consumption}

\subsubsection{Introduction}

The increasing demand of the data centers work has created an obtrusive impact on the energy consumption from power grid. The efficiency of data centers has become a topic of concern as the densely packed, energy intensive computer equipment inside a data center are creating power demands that are much higher than those of a standard residence or commercial office building [34]. In fact, data centers can be 40 times more energy intensive than a standard office building and require higher levels of power and cooling [35]. Furthermore, direct power consumption for these data com facilities is increasing due to growing demand for the services they provide, particularly internet and intranet services [36].

A major portion of US electricity supply is being utilized by the data centers. The report by US Environmental Protection Agency (EPA) dated: August 2, 2007, on server and data center energy efficiency to Congress, summarizes summarize that US server and data center sector used 61 terawatt hours (TW h) of electricity in 2006 (double the amount consumed in 2000). In 2006, this 61 TW h of electricity represented $1.5 \%$ of total US electricity consumption and cost $\$ 4.5$ billion [3]. Current efficiency scenario states, US energy consumption by servers and data centers could nearly double again by 2012 to 107.4 TW h or $\$ 7.4$ billion annually [37].

According to a survey conducted by the Association for Computer Operation Managers (AFCOM) and Inter Unity Group, data centers are growing larger in size, consuming more electricity and dissipating more energy in form of heat. Data center power requirements on an average are increasing by $8 \%$ per year, and $20 \%$ per year in the largest centers [38]. This 
results in problems with maintaining temperature of the devices and system. As processor manufacturers such as Intel, AMD, IBM, and others continue promising follow on Moore's Law doubling the number of transistors on a piece of silicon every 18 months - the resulting power density increase within the chips leads to dramatically rising temperatures inside and around those chips [39]. As servers become more power dense, more kilowatts are required to run and to cool them. Of the total power consumed by a typical datacenter, about half is attributed to conventional cooling [36].

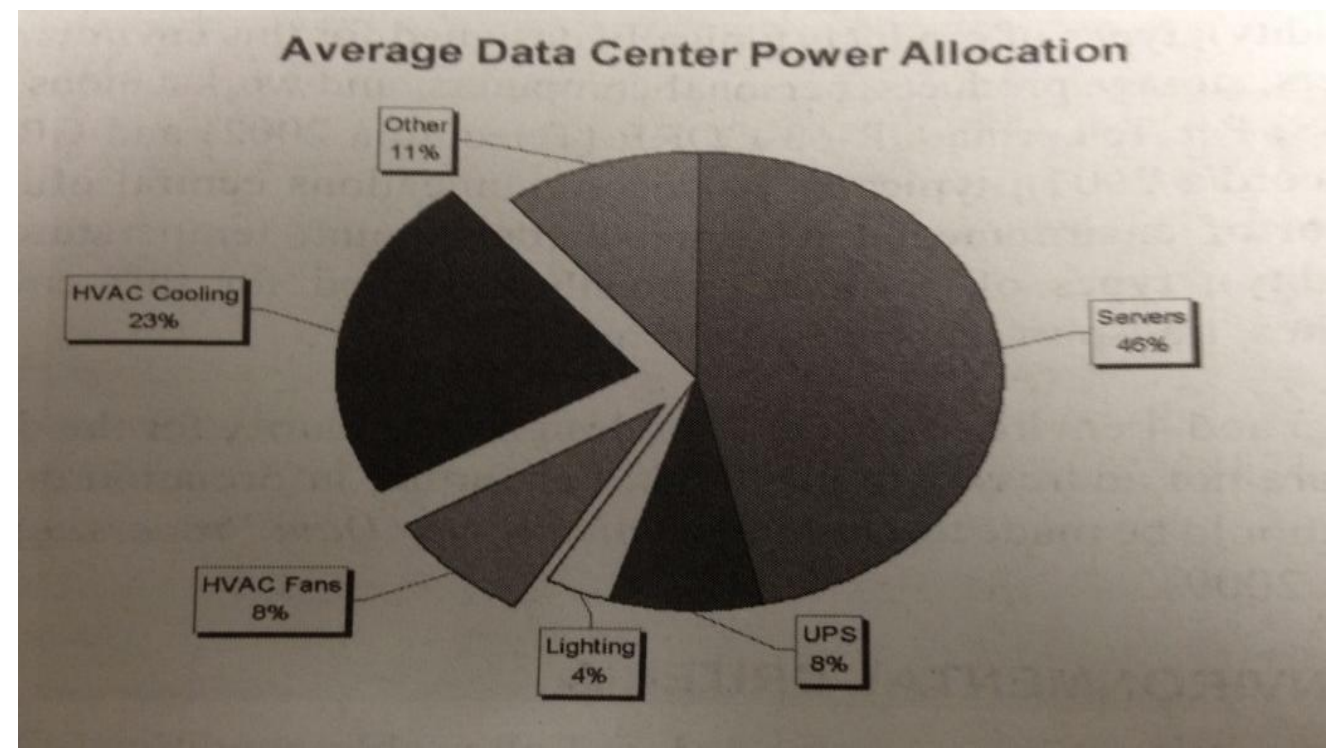

Figure 3.1: Energy Consumption Distribution of Data Center [4]

\subsubsection{Objective and Approach}

Cooling of data centers using standard electrically-driven vapor compression CRAC units is responsible for consuming up to $50 \%$ of power that a typical data center uses. Since these CRAC units consumes significant portion of grid energy so, the primary objective is to reduce the grid power consumption for the cooling system in data center.

Use of traditional cooling methods for data centers, such as computer room air conditioning units (CRAC), has resulted in the consumption of substantial amount of data center energy. Where applicable, the most common and less expensive techniques, air and liquid 
cooling are used over compressor based technology, such as CRAC units. Because of the less amount of energy required to run these cooling systems. Liquid cooling most commonly use water as a primary refrigerant to lower down the server temperature. High density servers require large volume of water to cool the servers to regulate their normal performance. In addition, little literature review says that according to a US geological survey, 39 percent of the water is consumed for power production alone in the United States. One method of conditioning outside air is to use indirect evaporative cooling system. Indirect evaporative cooling uses heat exchanger to cool the ambient air. The ambient air never comes in contact the coolant directly. It lowers the ambient air temperature sensibly keeping the relative humidity constant unlike direct evaporative cooling. Most commonly water has been used as coolant in both direct and indirect evaporative cooling system. In this study contains use of another coolant known as micro-encapsulated phase change slurry. It is an aqueous dispersion of a microencapsulated phase-change material.

\subsection{System Description and Configuration}

Phase change materials have been investigated over the years as thermal energy storage for cooling and heating applications. These researches have also investigated encapsulated PCM in different sizes in order to maximize its thermal properties in various heat transfer applications. They are currently in use in systems like PCM heat transfer fluid in heat exchangers, buildings, as well as storage facilities. However, little research effort has investigated on its use as a pumpable heat transfer fluid.

\subsubsection{Description of Experimental Setup}

The first set-up is a system comprising of a cooling tower, air handler and coolant circulating pipes, as shown in the schematic: 


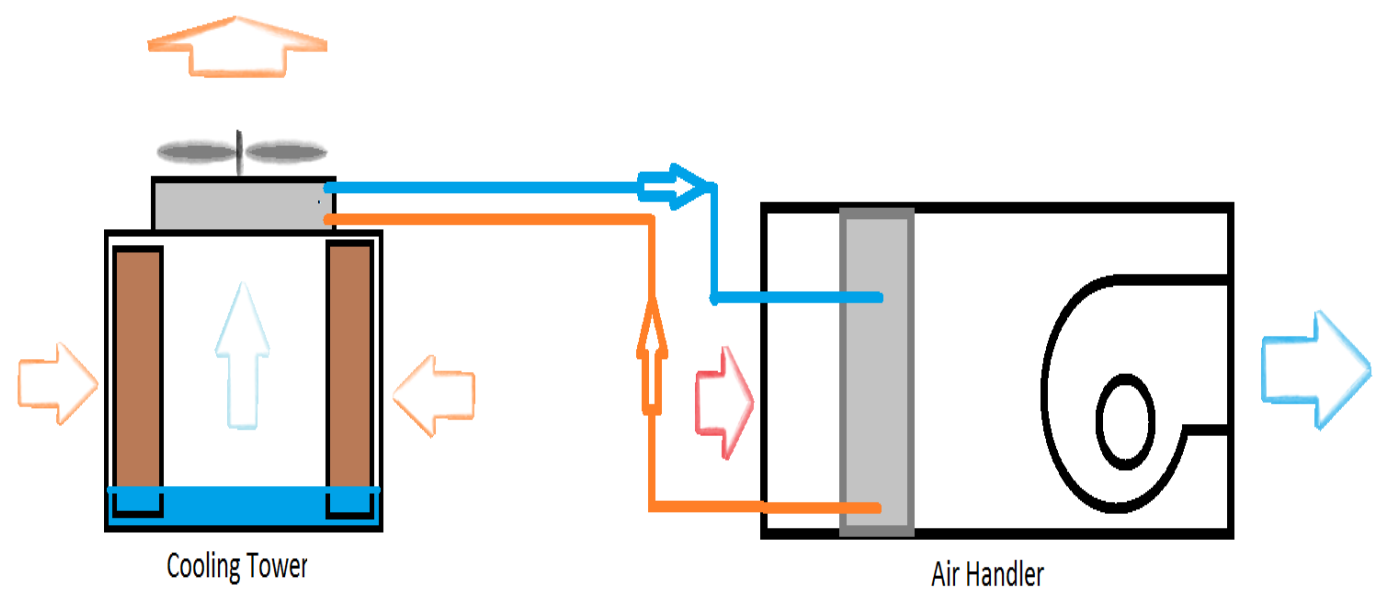

Figure 3.2: Schematic of the Experimental Setup

\subsubsection{Methodology of the Experiment:}

1. Ambient air is allowed to enter the rectangular duct.

2. Ambient air is made to pass through the external gas burner; to make its temperature is above the melting point of phase change material.

3. After passing over the burner and attaining the desired temperature, it made to pass over the cooling coils

4. The copper cooling coils contains microencapsulate phase change slurry which is circulated with the help of centrifugal pump

5. Now the cold air enters the supply fan area, and gets pushed down to the supply air openings to the container.

\subsubsection{Air Handler Unit}

A direct drive propeller wall fan was used to push cool supply air into the room. It has a volume flowrate of $9003 \mathrm{ft} 3 / \mathrm{m}$ and power of $2.32 \mathrm{bhp}$. 


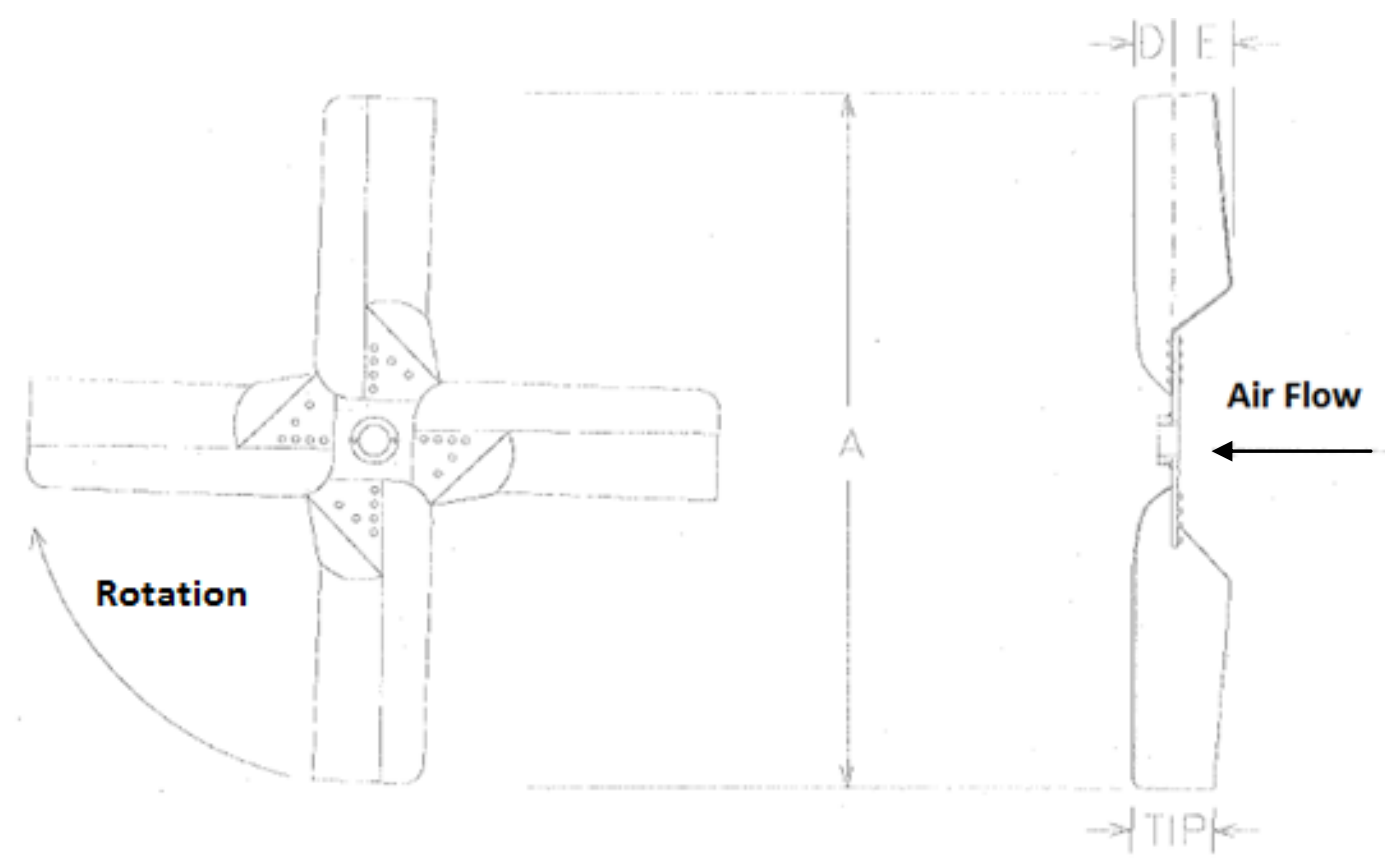

Figure 3.3: Direct Drive Wall Fan

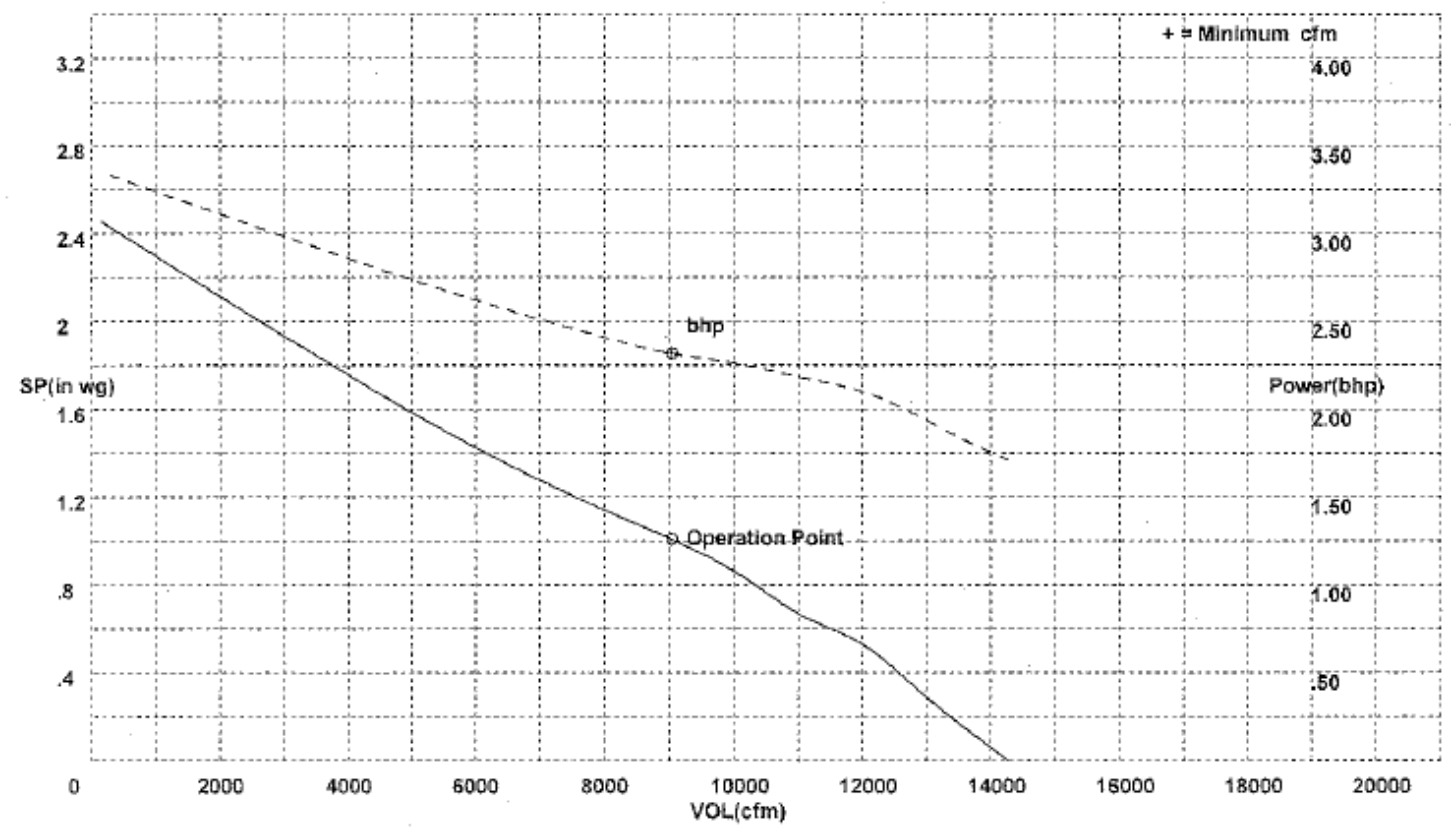

Figure 3.4: Fan curve for Air Handler 


\subsubsection{Cooling Tower}

The cross flow design cooling tower is used to cool the slurry after it takes up heat from the ambient air in heat transfer section. Cross flow type cooling tower is chosen. In this type of cooling tower the air flow pattern is perpendicular to the flow of coolant as shown in the figure below. Outside air enter the evaporative cooling pads from one or more vertical faces. Water is allowed to flow over the cooling pad through distributor in the direction of gravity. It uses the principle of direct evaporative cooling, which cools the air by reducing its dry bulb temperature and increases its specific humidity. Lastly, a fan forces the air out into the atmosphere. Gravity distributes the water through the evaporative cooling pad

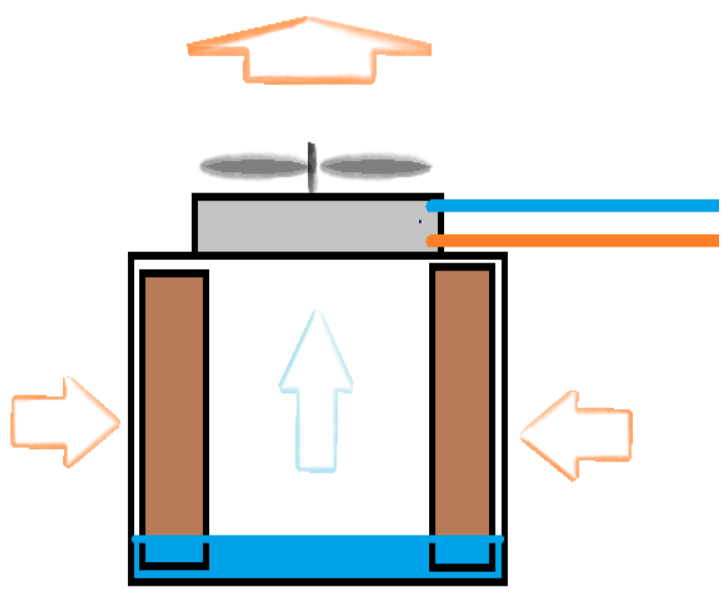

Figure 3.5: Schematic of Cooling Tower

\subsubsection{Advantages of the Cross Flow Design:}

- Gravity water distribution reduces pumping power and provides natural maintenance

- Non-pressurized spray provides uniform water flow over pad

- Relatively cheap and less expensive, mainly due to small pump 


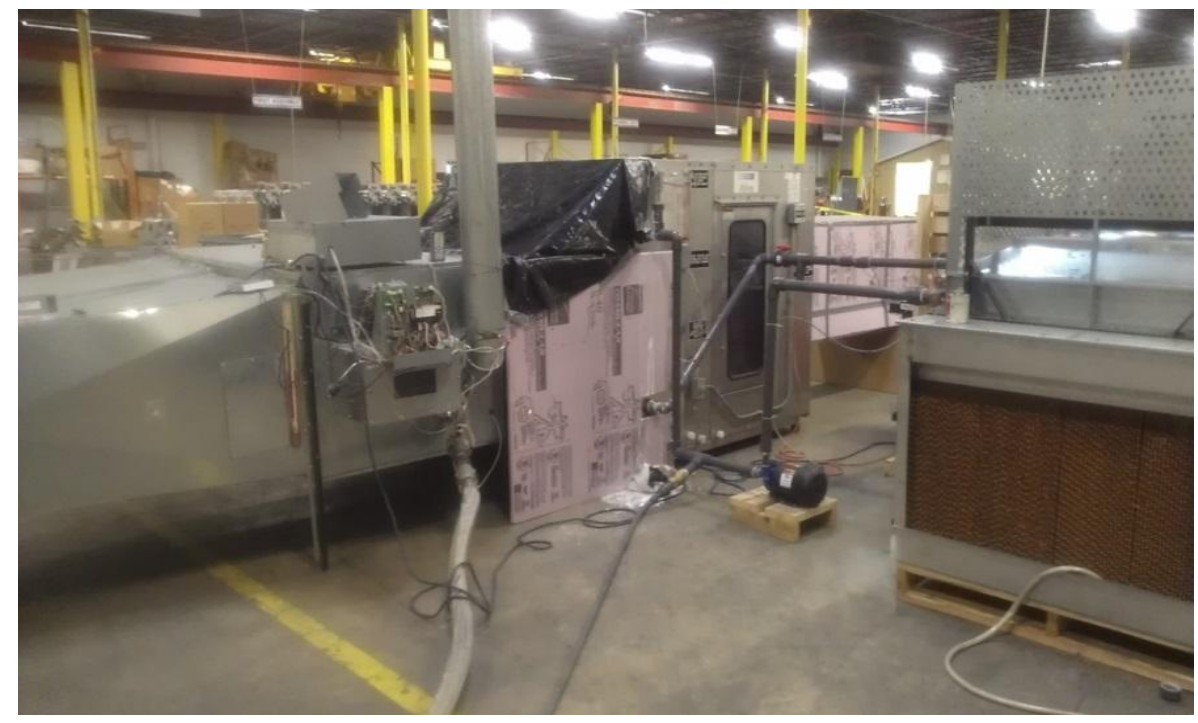

Figure 3.6: Cooling Tower used in Experiment

\subsubsection{Disadvantages of the cross flow design:}

- More prone to freezing than counter flow designs

- Variable flow is useless in some conditions

\subsubsection{Centrifugal Pump}

Cast Iron centrifugal pump and electric motor assembly was used in the experiment to circulate water and slurry. The pump has following specification: 1/2 horsepower, three phase totally enclosed fan cooled ac electric motor. 1 inch Inlet x 3/4 inch outlet NPT ports. Variable frequency drive was used vary the flow rate of the water and or slurry inside the cooper coils. 


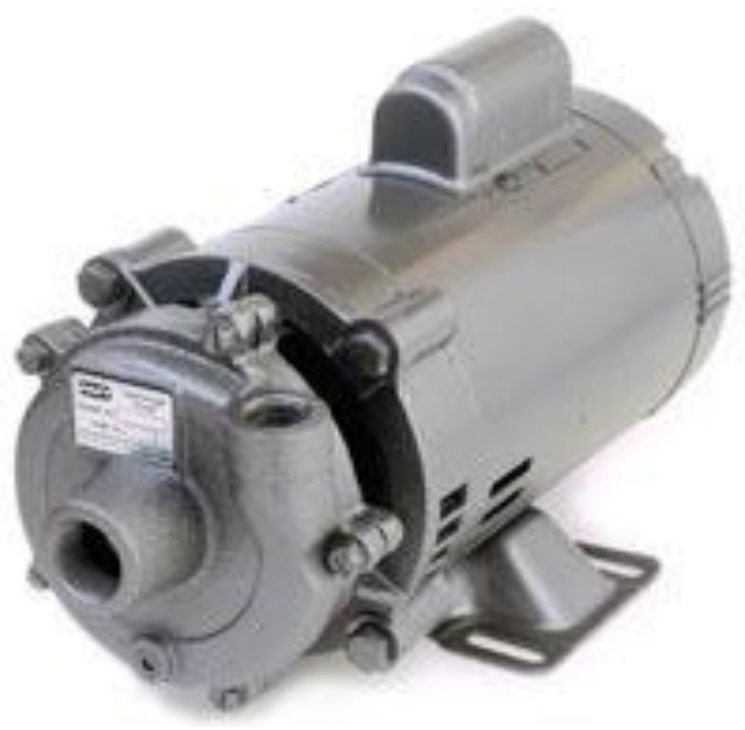

Figure 3.7: Centrifugal Pump [40]

\subsubsection{Microencapsulated Phase Change Slurry}

The dispersion type mPCM Micronal 5007-X was first used in the experiment. The following figure below on the left shows in general the appearance of microencapsulated phase change slurry and its image under scanning electron microscope on the right. Followed by the figure 3.7 in the red outline are the specifications of the BASF Micronal 5007-X used initially. This type of mPCM was not found compatible with the system. It resulted in clogging and agglomeration problems. In order to resolve this new kind of slurry (Micronal 5045-X) was used for final experiments. 


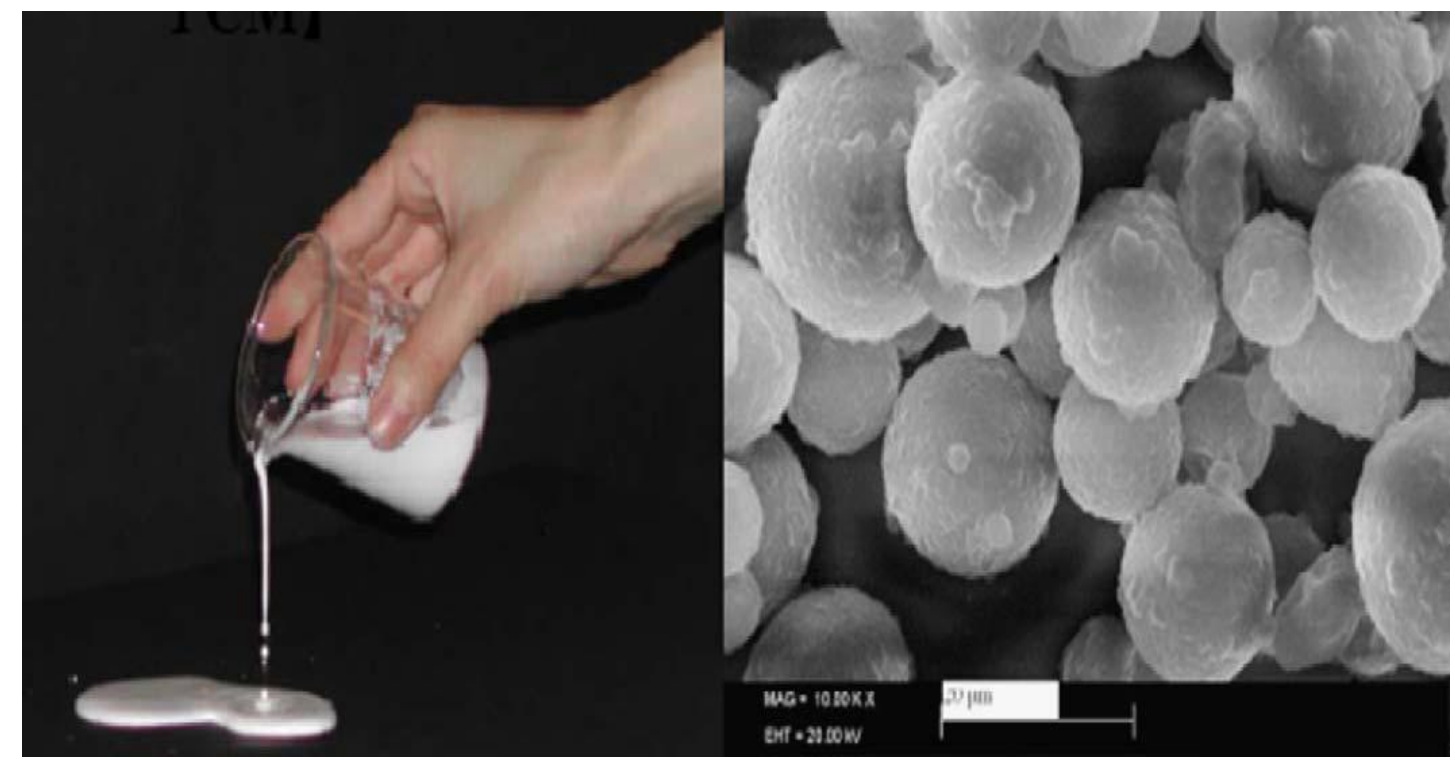

Figure 3.8: General Appearance of Phase Change Slurry and SEM Image [41]

\begin{tabular}{|c|c|c|c|c|c|c|c|c|c|c|c|}
\hline \multirow[t]{2}{*}{ PRODUCT } & \multicolumn{3}{|c|}{ APPLICATION } & \multicolumn{8}{|c|}{ CHARACTERISTICS } \\
\hline & 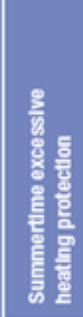 & 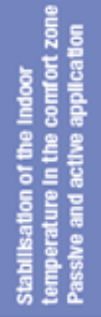 & 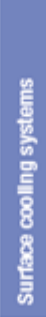 & $\frac{8}{\frac{8}{2}}$ & 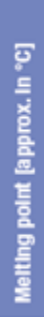 & 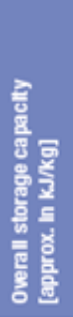 & 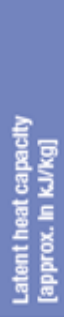 & 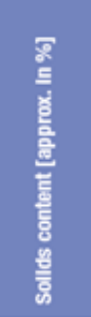 & 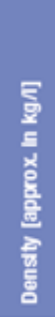 & 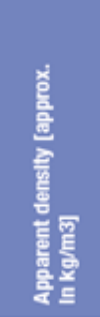 & 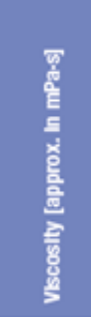 \\
\hline MIcronal $^{\bullet}$ DS 5000 & $\mathbf{a}$ & & & Dispersion & 26 & 59 & 45 & $42-44$ & 0.98 & & $200-600$ \\
\hline MIcronal $^{\bullet}$ DS 5001 & 물 & & & Powder & 26 & 145 & 110 & & & $250-350$ & \\
\hline MIcronal $^{\bullet}$ DS 5007 & & = & & Dispersion & 23 & 55 & 43 & $42-44$ & 0.98 & & $200-600$ \\
\hline MIcronal $^{\bullet}$ DS 5008 & & a & & Powder & 23 & 135 & 100 & & & $250-350$ & \\
\hline MIcronal ${ }^{\bullet}$ DS 5030 & & & a & Dispersion & 21 & 51 & 37 & $42-44$ & 0.98 & & $200-600$ \\
\hline MIcronal $^{\bullet}$ DS 5029 & & & a & Powder & 21 & 125 & 90 & & & $250-350$ & \\
\hline MIcronal$^{\bullet}$ DS $5037 \mathrm{X}$ & घ & & & Dispersion & 26 & 59 & 45 & $42-44$ & 0.98 & & $200-600$ \\
\hline MIcronal $^{\circ}$ DS $5038 \mathrm{X}$ & घ & & & Powder & 26 & 145 & 110 & & & $250-350$ & \\
\hline MIcronal ${ }^{\bullet}$ DS $5039 \mathrm{X}$ & & $\mathbf{E}$ & & Dispersion & 23 & 55 & 43 & $42-44$ & 0.98 & & $200-600$ \\
\hline MIcronat $^{\circ}$ DS $5040 \mathrm{X}$ & & E & & Powder & 23 & 135 & 100 & & & $250-350$ & \\
\hline
\end{tabular}

Figure 3.9: Specifications of the Micronal 5007-X [42] 


\subsubsection{Micronal DS 5045-X}

Micronal $5045 \mathrm{X}$ is an aqueous, formaldehyde-free dispersion of a paraffin wax mixture encapsulated in highly cross-linked polymethylmeth-acrylatelt. It meets all suitable properties to be used as heat transfer fluid in decentralized air conditioners. Also, it offer low pumping cost and helps in reducing the pumping power due to small particle size of $1-3 \mu \mathrm{m}$ of mPCM. The thermal capacity of slurry with a solids content of $35 \%$ is around $70 \%$ higher than that of water in the $20-35^{\circ} \mathrm{C}$ temperature range. [42]

Table 3.1: Specifications of Micronal 5045-X [42]

\begin{tabular}{|l|l|}
\hline Solids content & ca. $44 \%$ in water \\
\hline $\mathrm{pH}$ & $7.5-8.5$ \\
\hline Dynamic viscosity & $<1500 \mathrm{mPa} . \mathrm{s}$ \\
\hline Density & $\leq 1 \mathrm{~g} / \mathrm{cm} 3$ \\
\hline Peak melting range & ca. $26-28^{\circ} \mathrm{C}$ \\
\hline Enthalpy of fusion (emulsion, liquid) & ca. $68 \mathrm{~kJ} / \mathrm{kg}$ \\
\hline Thermal capacity ${ }^{*}\left(20-35^{\circ} \mathrm{C}\right.$, liquid) & ca. $117 \mathrm{~kJ} / \mathrm{kg}$ \\
\hline Enthalpy of fusion (active solids) & min. $160 \mathrm{~kJ} / \mathrm{kg}$ \\
\hline $\begin{array}{l}\text { Thermal capacity } \\
\left(20-35^{\circ} \mathrm{C}, \text { solids) }\right.\end{array}$ & ca. $190 \mathrm{~kJ} / \mathrm{g}$ \\
\hline $\begin{array}{l}\text { Thermal capacity } \\
\left(20-35^{\circ} \mathrm{C} \text { at } 35 \%\right)\end{array}$ & $\begin{array}{l}\text { ca. } 106 \mathrm{~kJ} / \mathrm{kg} \text { (Slurry containing } \\
35 \% \text { solids })\end{array}$ \\
\hline $\begin{array}{l}\text { Thermal capacity relative to water } \\
\left(20-35^{\circ} \mathrm{C}\right)\end{array}$ & $\begin{array}{l}\text { ca. }+68 \% \\
(35 \% \text { solids })\end{array}$ \\
\hline Particle diameter & ca. $1-3 \mu \mathrm{m}$ \\
\hline
\end{tabular}

The following formula is provided by BASF Micronal that can be used to calculate the overall thermal capacity as a function of the solids content

$$
\begin{gathered}
\text { QPCM }=\text { mPCM } \times \Delta \mathrm{H}(\rightarrow \text { within } \Delta \mathrm{T}) \\
\text { Qwater }=\text { mwater } \times 4.21 \mathrm{~kJ} / \mathrm{kgK} \times \Delta \mathrm{T} \\
\text { Qtotal }=\mathrm{QPCM}+\text { Qwater } \\
\Delta \mathrm{H}=\text { approx. } 160 \mathrm{~kJ} / \mathrm{kg}=44 \mathrm{Wh} / \mathrm{kg}
\end{gathered}
$$




$$
\begin{gathered}
\mathrm{m}=\text { Mass fraction of } \mathrm{PCM} \text { or water } \\
\Delta \mathrm{T}=\text { Change in temperature }
\end{gathered}
$$

The chart below shows the enthalpy-temperature for the selected microencapsulated phase change slurry.

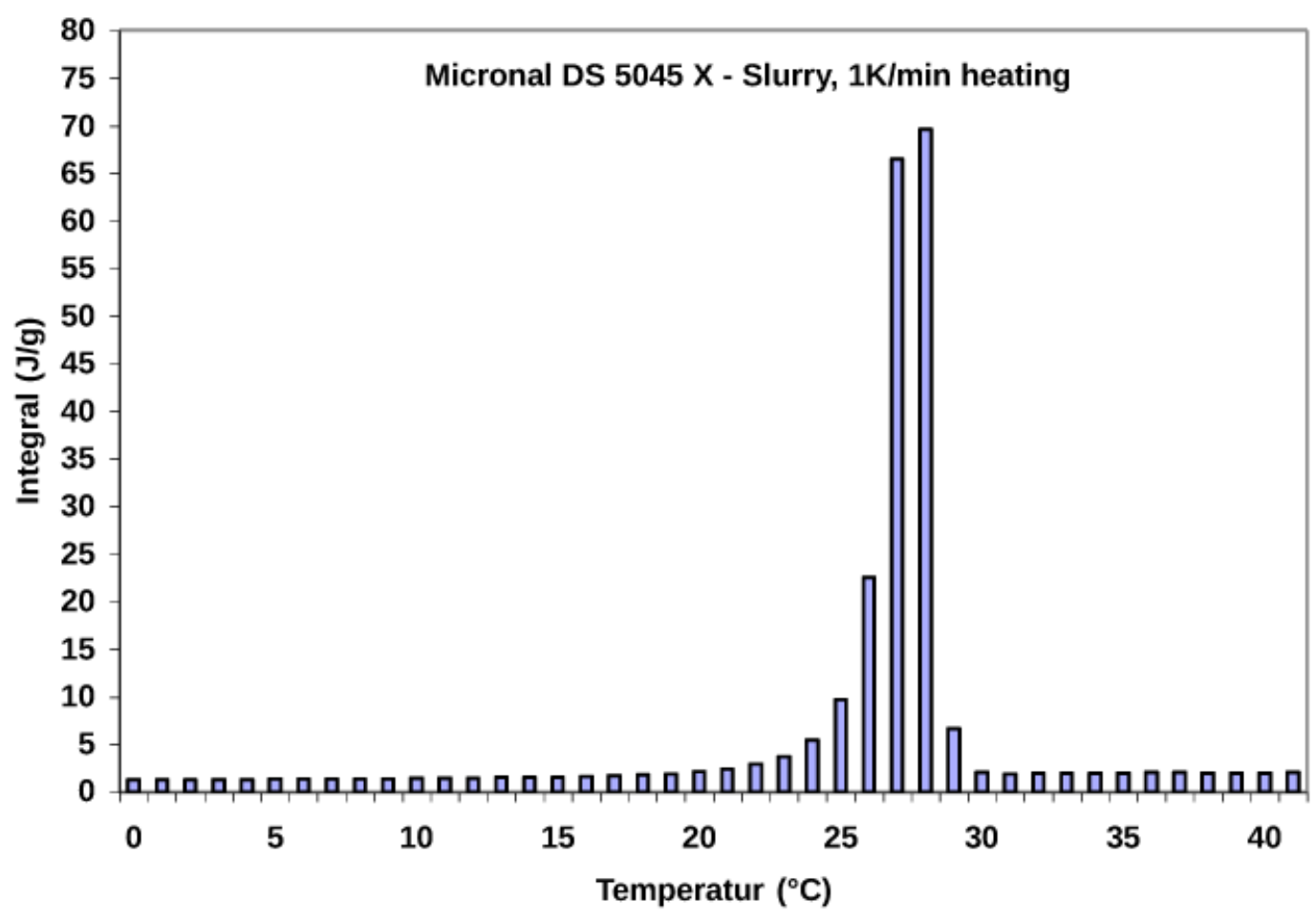

Figure 3.10: Enthalpy-Temperature graph [42] 


\section{CHAPTER 4}

\section{SCANNING ELECTRON MICROSCOPY FOR MICROENCAPSULATED PHASE CHANGE}

\section{SLURRY}

\section{$\underline{4.1 \text { Scanning Electron Microscopy Working (SEM) }}$}

SEM is an instrument that produces a largely magnified image by using electrons instead of light to form an image. A beam of electrons is produced at the top of the microscope by an electron gun.

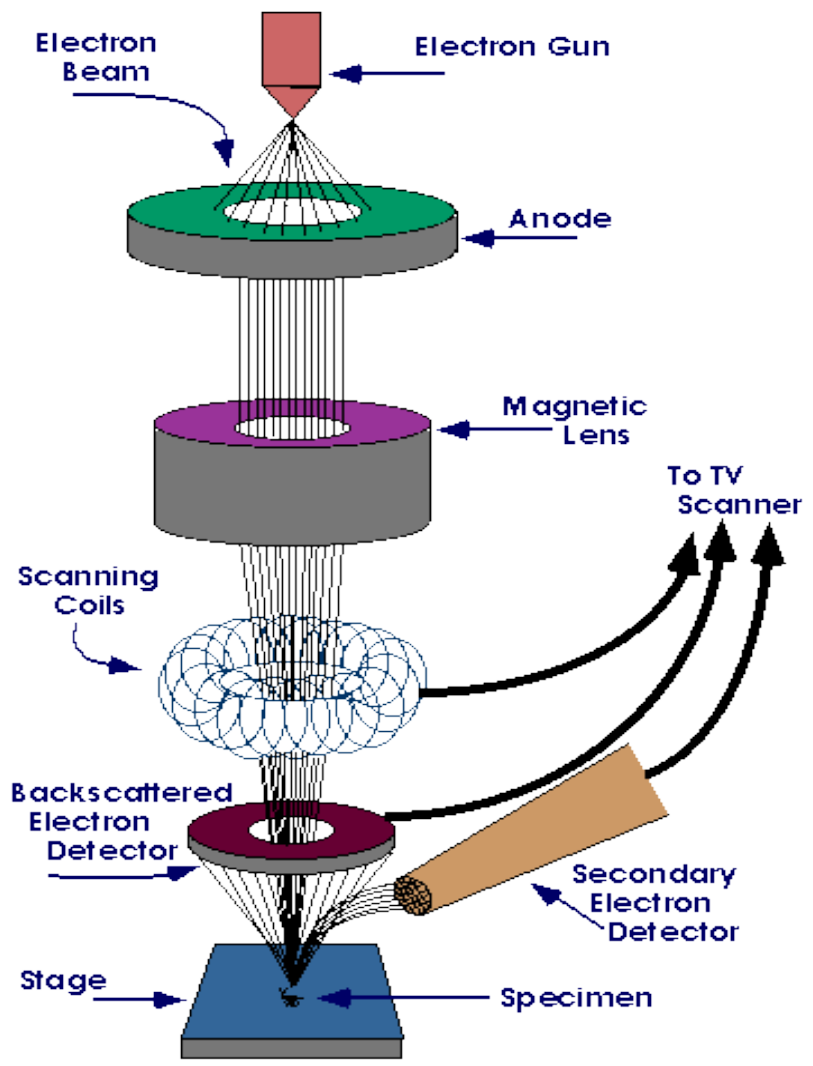

Figure 4.1: Working of SEM [43] 
The electron beam follows a vertical path through the microscope, which is held within a vacuum. The beam travels through electromagnetic fields and lenses, which focus the beam down toward the sample. Once the beam hits the sample, electrons and X-rays are ejected from the sample. SEM utilizes vacuum conditions and uses electrons to form an image all water must be removed from the samples because the water would vaporize in the vacuum.

\subsubsection{SEM Sample Preparation for mPCS}

No special preparation is required for SEM If the sample is conductive in nature. Since the slurry is non- conductive in nature the sample needs to be made conductive by covering it with a thin layer of conductive material i.e. either gold or silver. The process of depositing thin layer of metal on the nonconductive sample is known as sputtering operation. The device used for sputter coating is called a "sputter coater. Figure below shows the pictorial view of sputter coating machine.

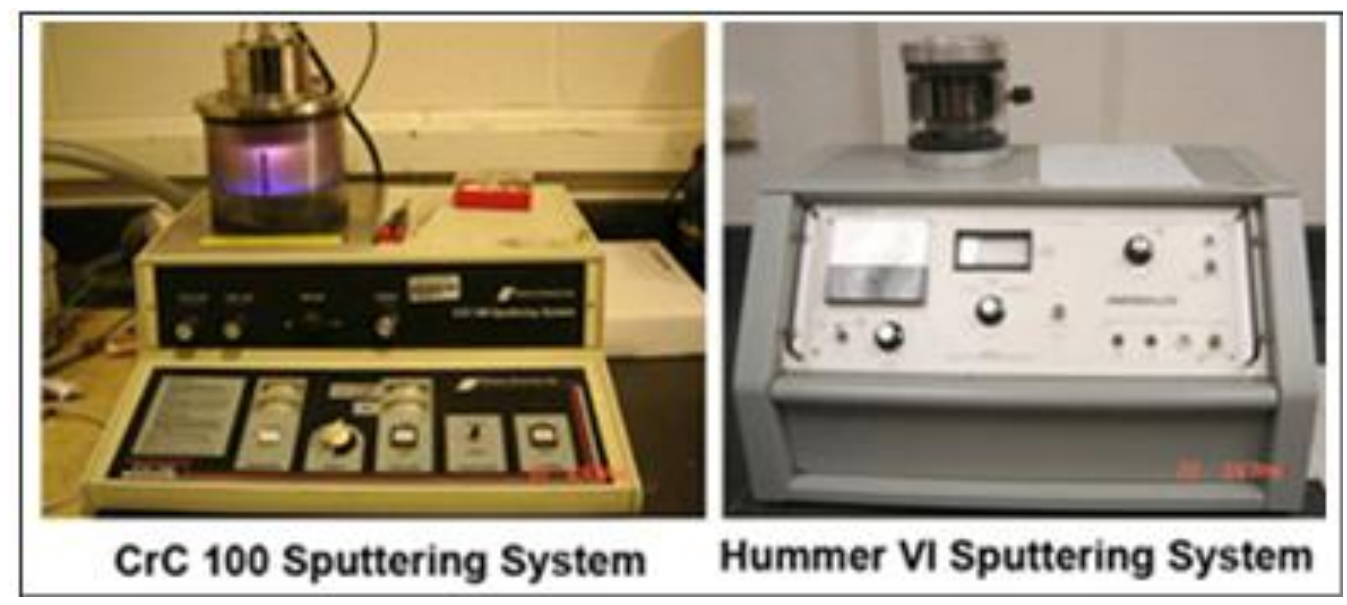

Figure 4.2: Sputter Coating Machines

The sputter coater uses an electric field and argon gas. The sample is placed in a small chamber that is at a vacuum. Argon gas and an electric field cause an electron to be removed from the argon, making the atoms positively charged. The argon ions then become attracted to a negatively charged gold foil. The argon ions knock gold atoms from the surface of the gold foil. These gold atoms fall and settle onto the surface of the sample producing a thin gold 
coating [43]. For analyzing samples of mPCS 10-15 seconds of sputter coating is enough. If coated for more time, the samples particles will not be visible under microscopic view.

\subsubsection{Stub}

Stub is the aluminum base on which liquid $\mathrm{mPCM}$ are placed. The samples need to be dried before analyzing in SEM, because in vacuum water will evaporate. The following figure shows the different mPCS samples on the stub.

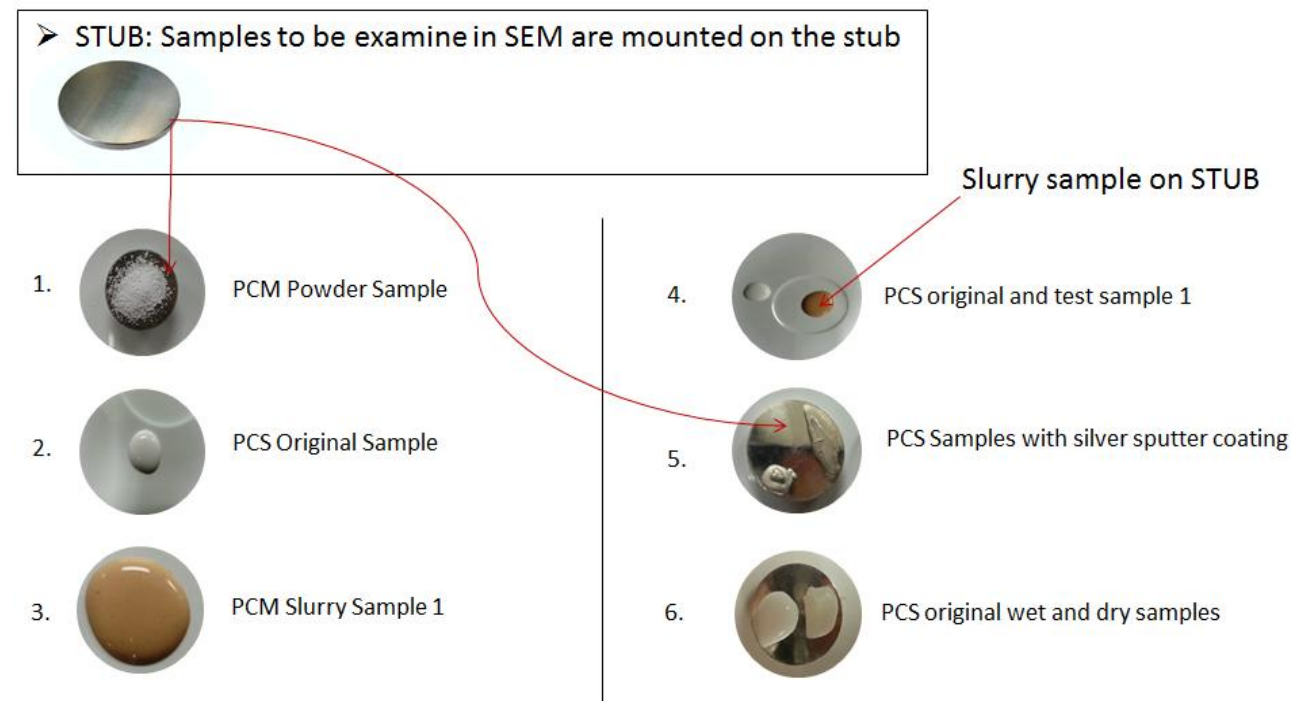

Figure 4.3: Dry and Wet mPCS samples on Stub
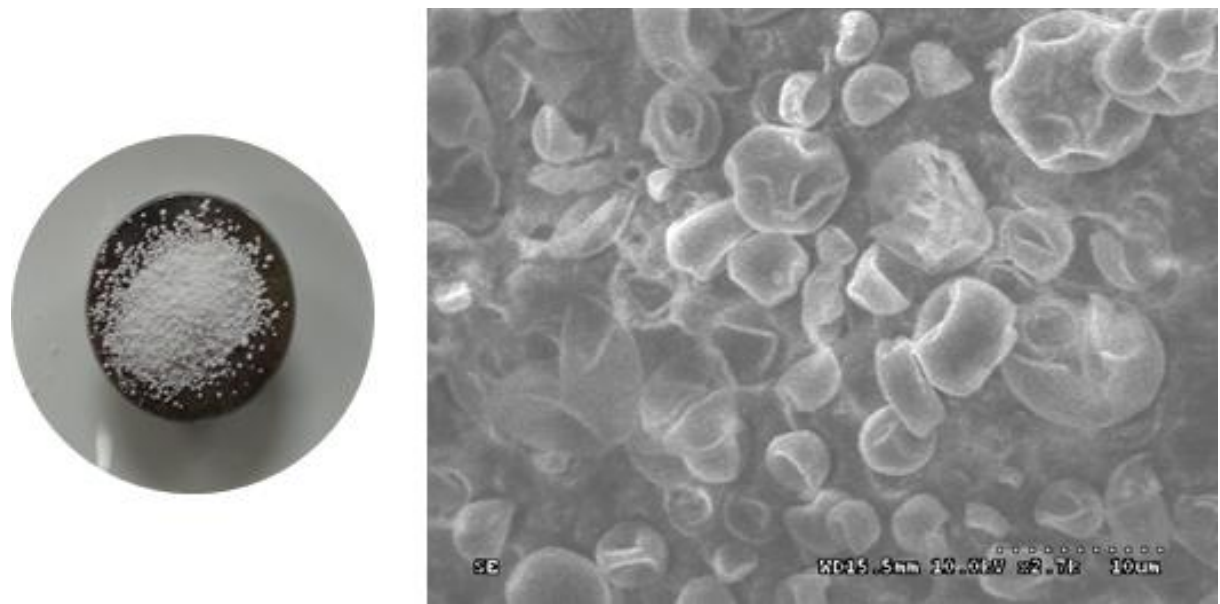

Figure 4.4: The general appearance of the powder mPCM capsule on the left and its SEM image on the right at UT Arlington 
CHAPTER 5

\section{EXPERIMENTAL RESULTS AND DISCUSSIONS}

\section{$\underline{5.1 \text { Initial Results }}$}

Initial testing was done on a large industrial size test rig. The figure below shows the basic schematic of the system. The stainless steel unit contains:

- Direct drive propeller wall fan with volume CFM of 9033 and power of 2.2 bhp

- Cross flow type cooling tower

- Centrifugal Pump with 1/2 horsepower, 1 inch Inlet x 3/4 inch outlet NPT and 3 phase totally enclosed fan cooled electric motor

- PVC Pipes

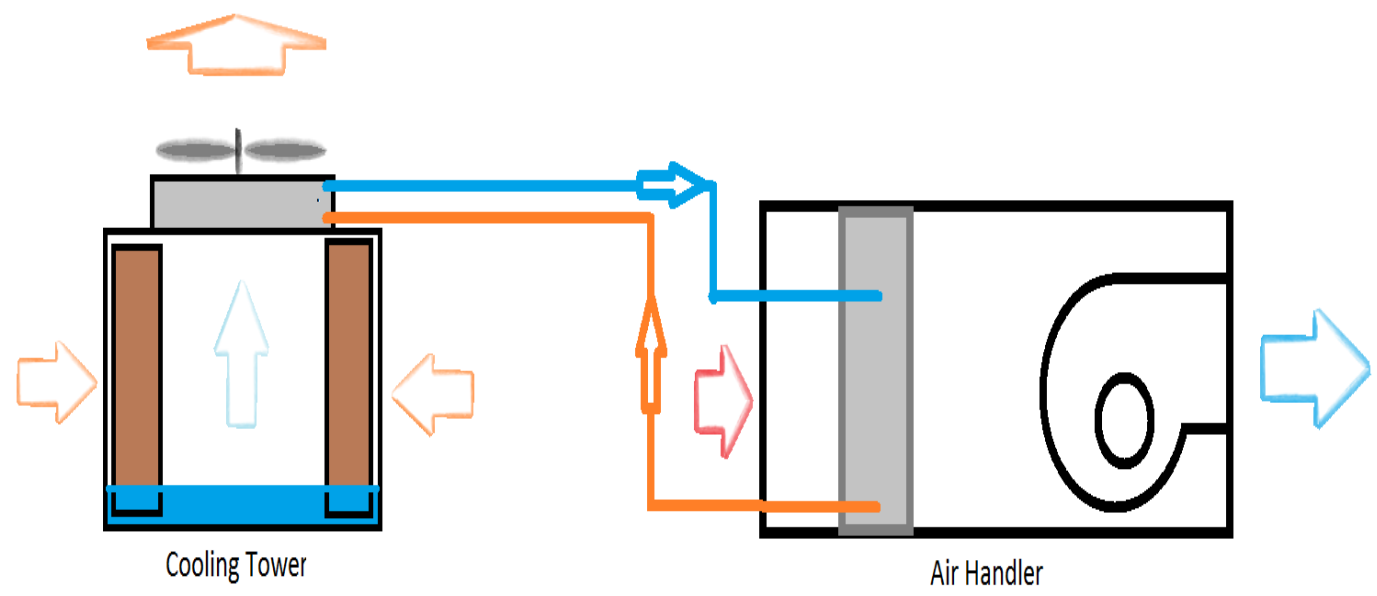

Figure 5.1: Initial Test Setup

Testing was initially started with water and air to make a baseline case. In addition only $4.8 \%$ of PCM solution was used for testing after water and air. Figure 5.1 shows the graph between preliminary testing for water, air and 4.8\% PCM solution. Uniform temperature difference across the heat source and the heat extraction unit is maintained by varying the flow 
rate. The intent is to obtain a temperature difference equivalent to the mPCM transition temperature range. Curve from water and air testing shows presence of hysteresis in testing. In general, the performance with water and air increases with flow rate. No significant results on cooling side were obtained with $4.8 \% \mathrm{PCM}$ testing but the performance was greatly affected by the wet bulb temperature as shown in graph.

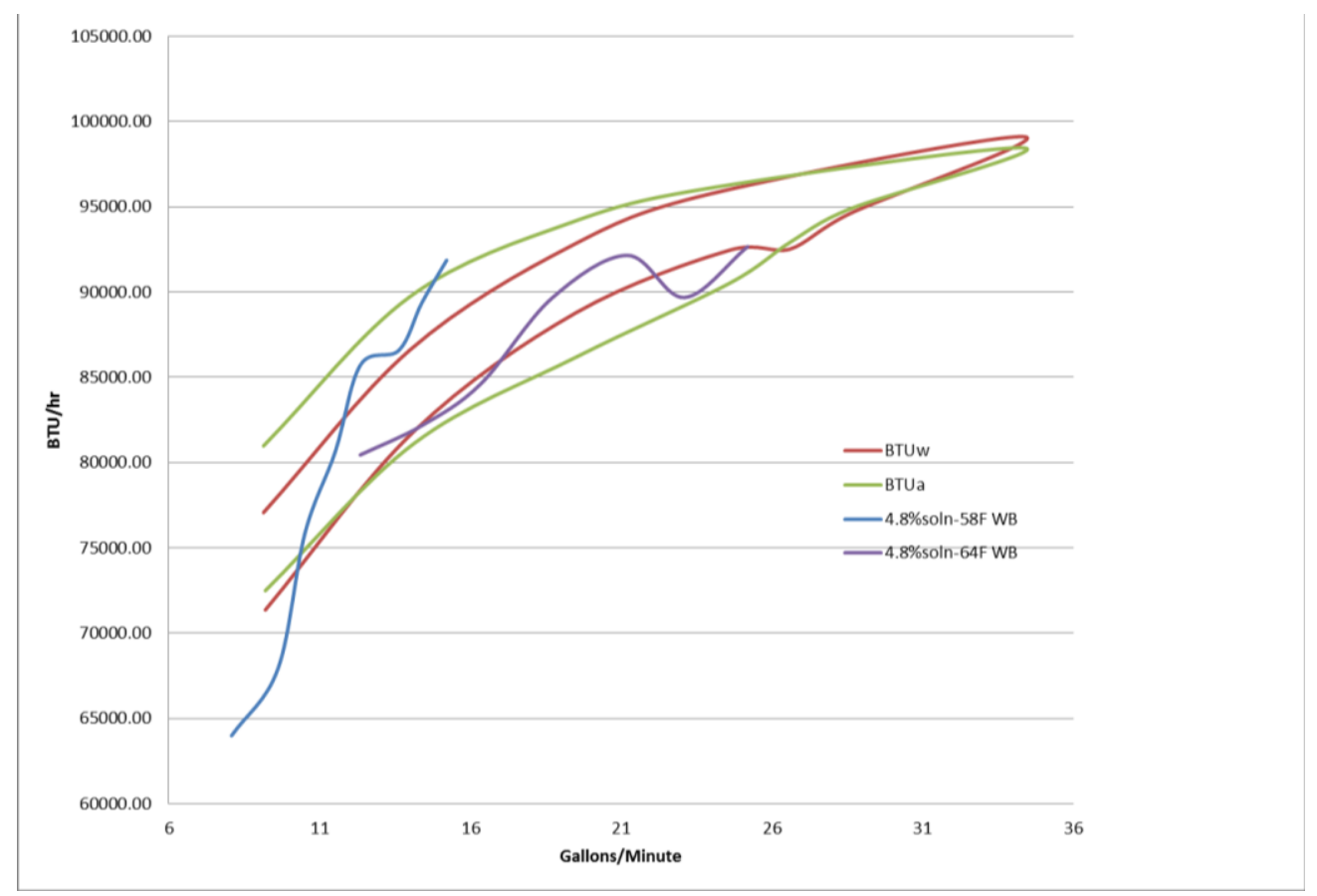

Figure 5.2: Preliminary Testing with 4.8\% PCM Solution

After approximately 24 hour time, the pipes of the system were found clogged with PCM solution. The possible reasons for cogging and agglomeration could be either non compatibility of the type of pump selected or due to non - compatible material used in the piping. Also, it has been shown that mPCM can be stabilized with certain nucleating agent to mitigate the damage to the carrier shell when used as a pumpable fluid. In order to figure out the possible reasons of clogging and agglomeration the durability analysis was done. The figures below show the images of clogged slurry inside the circulating pipes. 


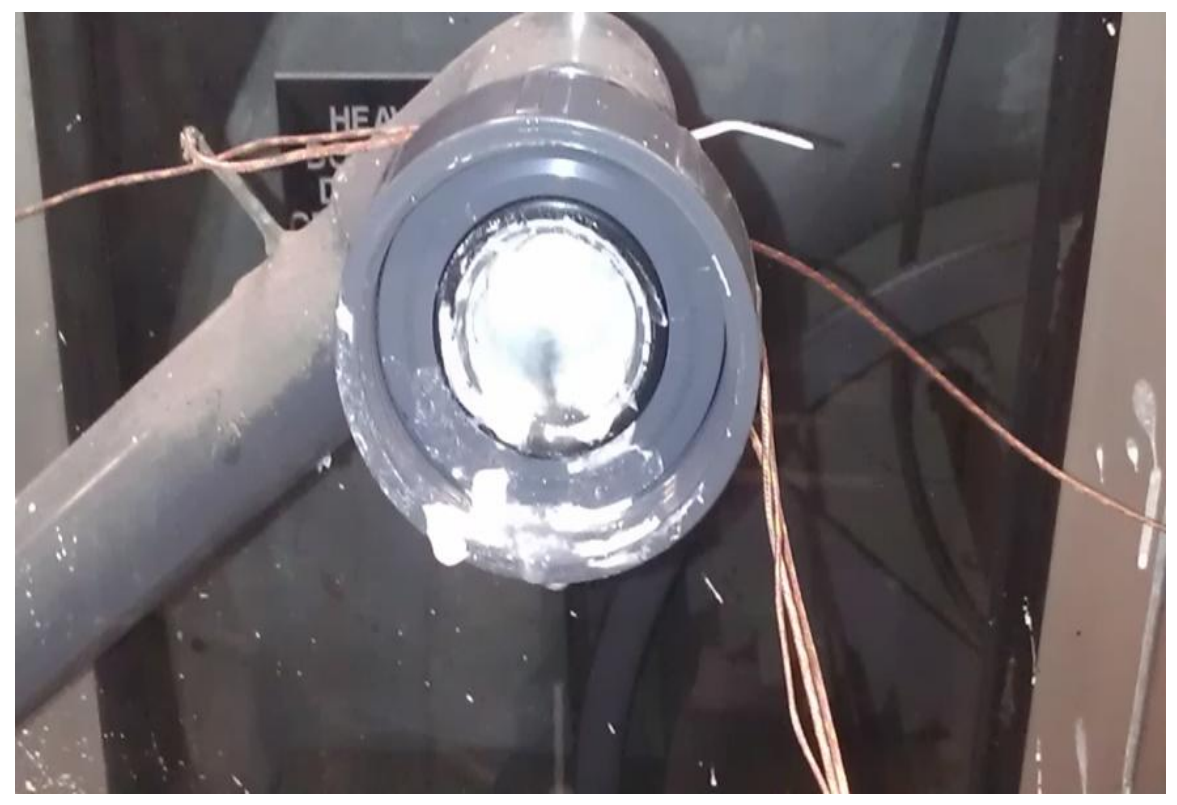

Figure 5.3: Clogged slurry inside the circulating pipes

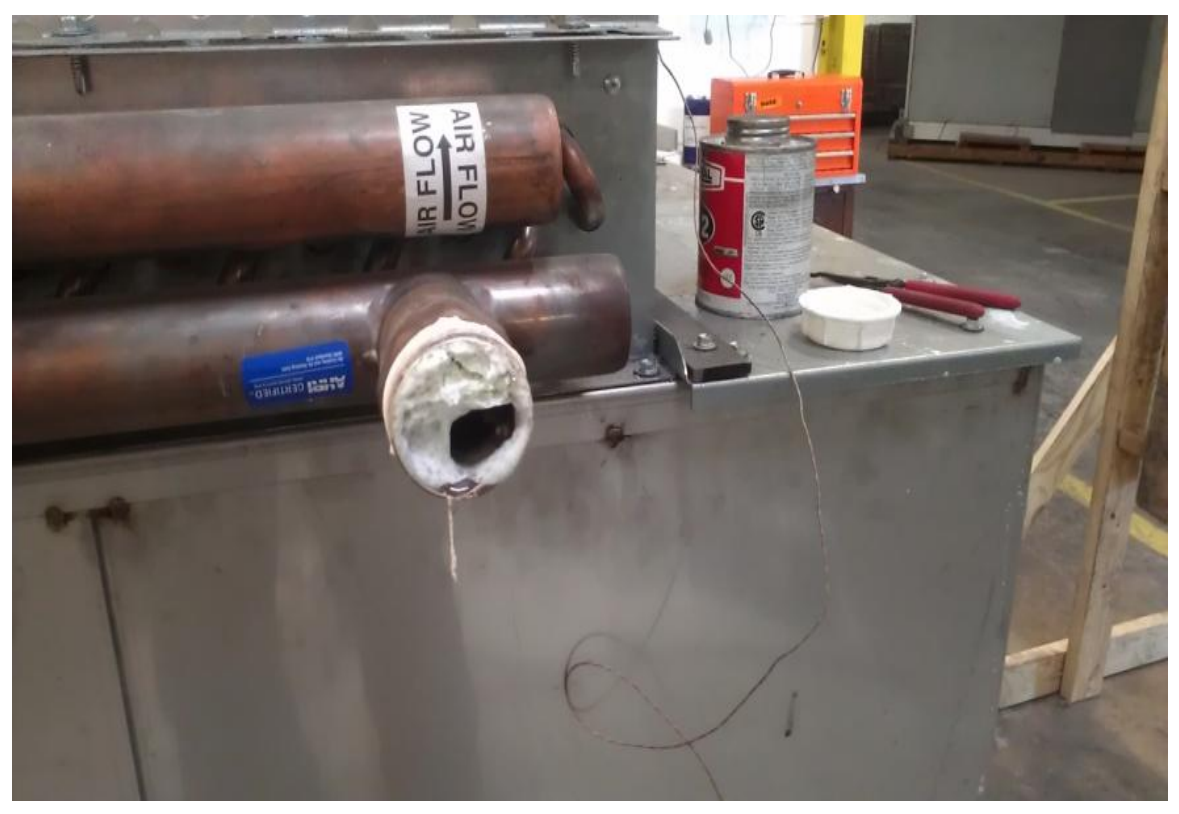

Figure 5.4: Clogging in the pipe at the outlet of cooling Tower In order to identify the possible causes of clogging durability analysis was done. 


\section{DURABILITY ANALYSIS}

\subsection{Closed Loop Testing Rig}

A set-up was built to identify the clogging problems of the microencapsulated phase change slurry (mPCS) flowing through a circular tube in the initial testing. Figure below shows the schematic of the test setup. It is a closed circuit that contains a heat source, centrifugal pump, expansion tank, heat extraction unit (Ice bath). Distilled water was introduced into the system as the heat transfer fluid initially and validation the setup was done by comparing the results with theoretical values.

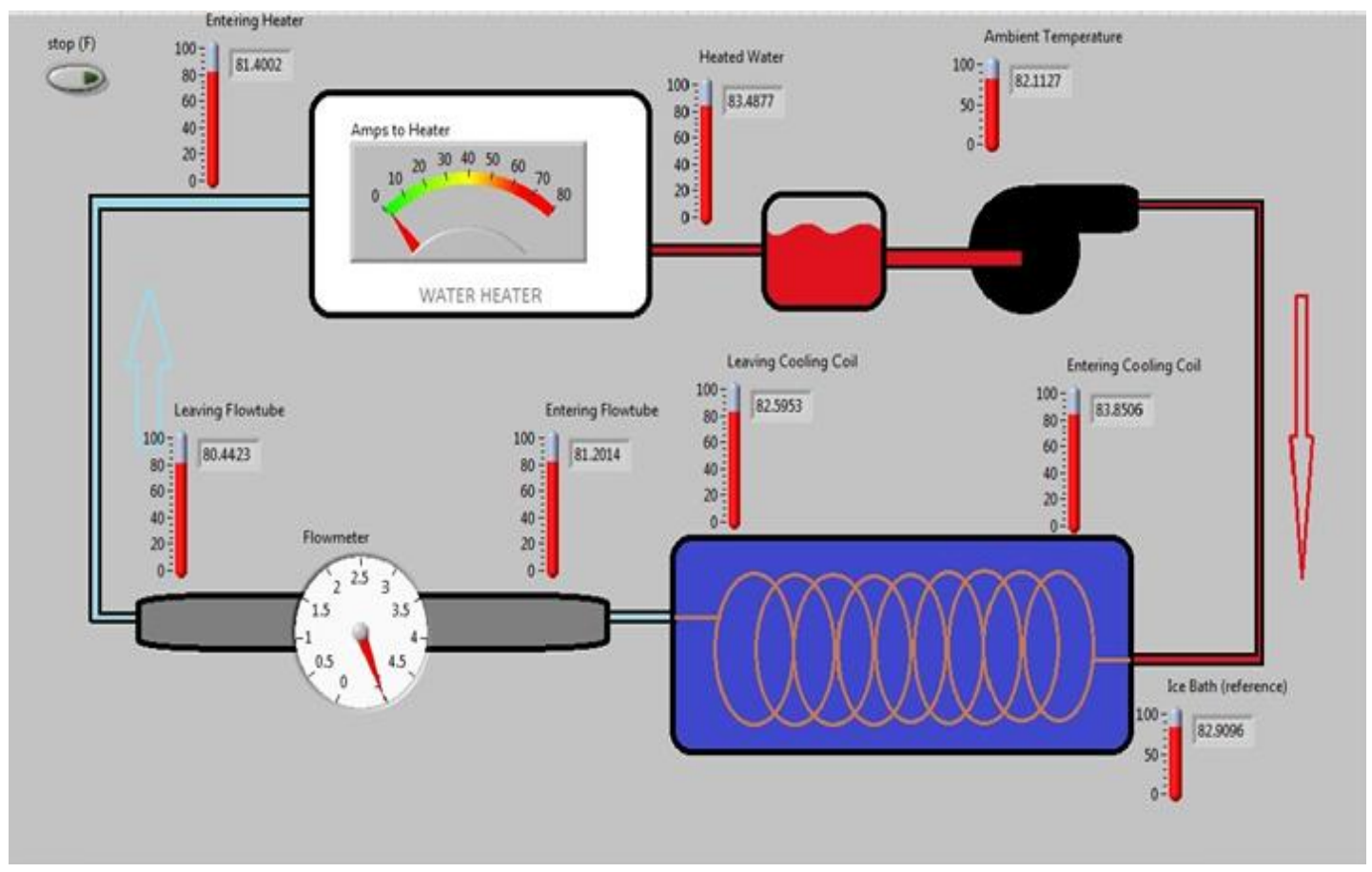

Figure 5.5: Schematic for mPCS Durability Analysis

The mPCS is pumped from the heater section and flows through the loop. The slurry proceeds to the heat transfer section, where heating elements provide uniformly distributed heat flux. Leaving the heat transfer section, the mPCS returns to the ice bath where it rejects its heat; passing through a thermal equilibrium section. The mass flow is measured by an electromagnetic flow meter installed in the piping. $T$ type thermocouple with temperature range of $-250^{\circ} \mathrm{C}$ to $350^{\circ} \mathrm{C}$ and limits of error $1.0^{\circ} \mathrm{C}$ or $0.75 \%$ were installed to measure the 
temperature of the slurry at different locations; temperature at the inlet and at the outlet of the heat transfer section and ice bath. The heater was connected to the AC power supply through a phase angle electronic regulator, which varied the voltage and therefore varied the heating power provided to the heat transfer section. The heat flux provided to the heat transfer section guaranteed complete phase change of the $\mathrm{mPCM}$ dispersed in the slurry. The graph below shows that transition temperature for the slurry was obtained.

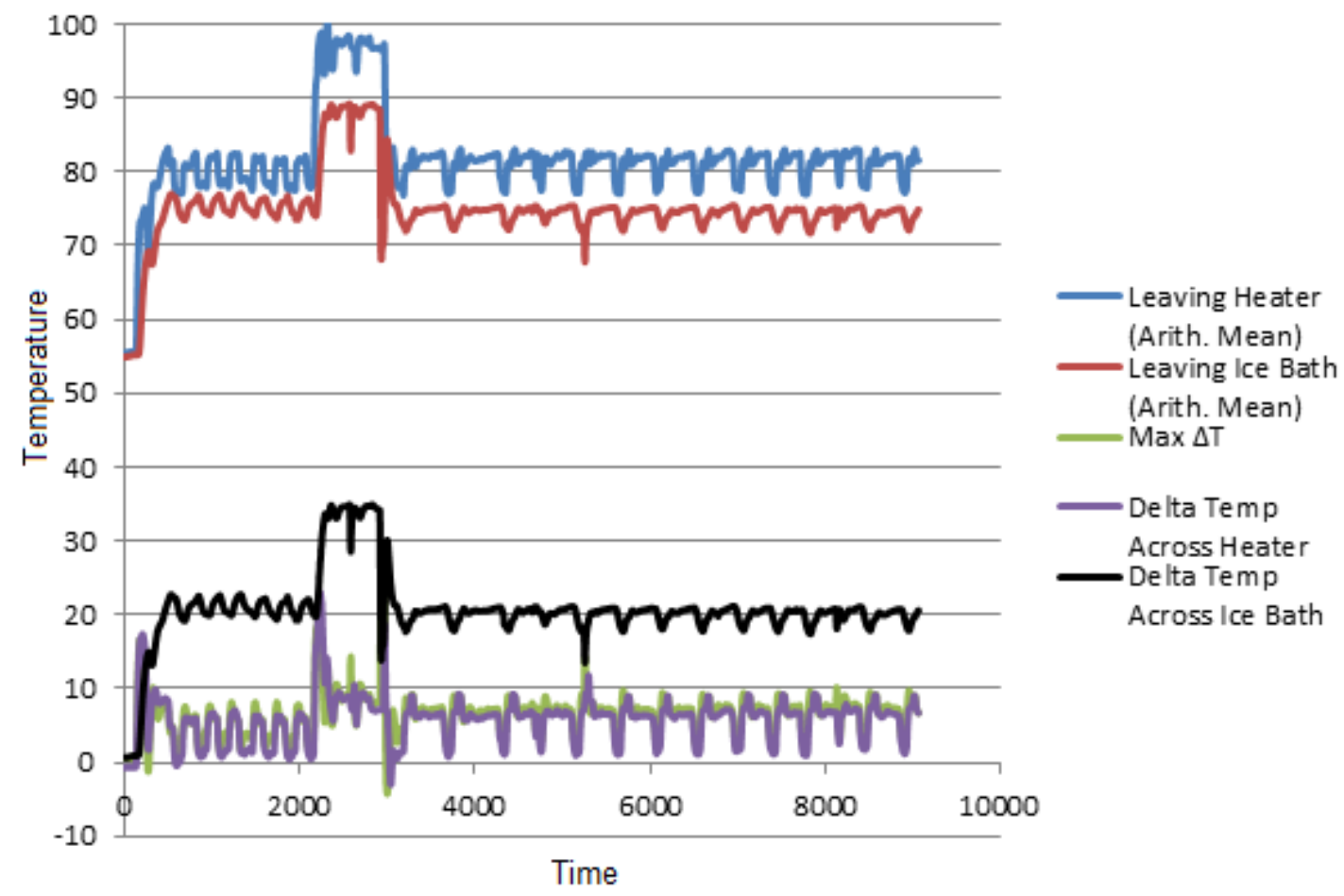

Figure 5.6: Transition and Crystallization Graph for mPCS

Heat losses were minimal, approximately $3 \%$, and therefore the heat transfer section was not thermally isolated. All measured data was recorded by using National Instruments data logger along and LabVIEW software. The tests were then repeated with the known temperature range and a solution containing $10 \%$ phase change slurry by weight. The pump was fitted on the hot side of the heater; this is done to ensure better cycling stability of the slurry. 


\subsubsection{Experimental Results}

The following experiment results in the clogging and agglomeration in the pipes after approximately 24 hours. The possible reasons encounter for clogging and agglomeration includes: Centrifugal pump selected for operation could be not compatible with

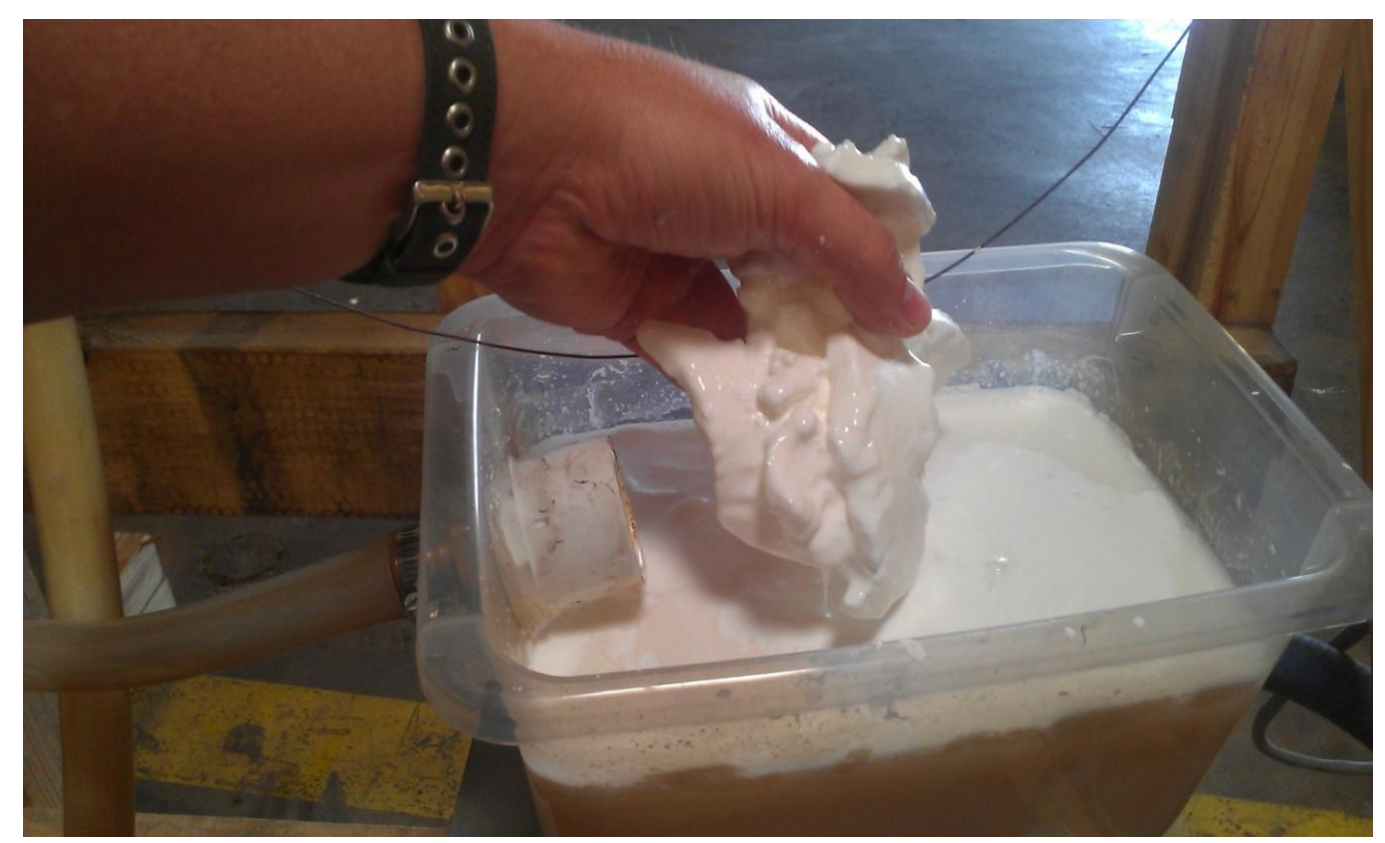

Figure 5.7: Agglomerate of mPCS in Expansion Tank

mPCS selected. That could have resulted in the breakage of shell material of microencapsulated phase change material. Also, the size of mPCM particles in the slurry affects the life of the mPCM particles. The non-compatibility of the any material used in the loop, most probably copper. The system was not fully air tight.

In order to resolve this issue, a new kind of mPCS (Micronal 5045-X) was selected and tested. The particle diameter of mPCM for this slurry ranges from $1-3 \mu \mathrm{m}$.

\section{$\underline{\text { 5.3 Pump Compatibility Test Setup for Micronal 5045- X }}$}

The stability of the micro-capsules in the slurry is greatly affected by high shear rates of the pump and pipe joints. Study from S. Gschwander et depict that after testing different pumps and pumping principles, centrifugal pumps were found able to pump the phase change slurry for a longer time without providing any damage to the shell material of the particles [26]. The 
intermediate goal of this testing to figure the stability of the new slurry obtained from BASF Micronal 5045-X. The figure below shows the basic test setup to conduct this experiment. The $U$ shape pipe was used to connect both inlet and outlet of the centrifugal pump with each other. T joint is provided at the outlet of the pump to fill in the slurry.

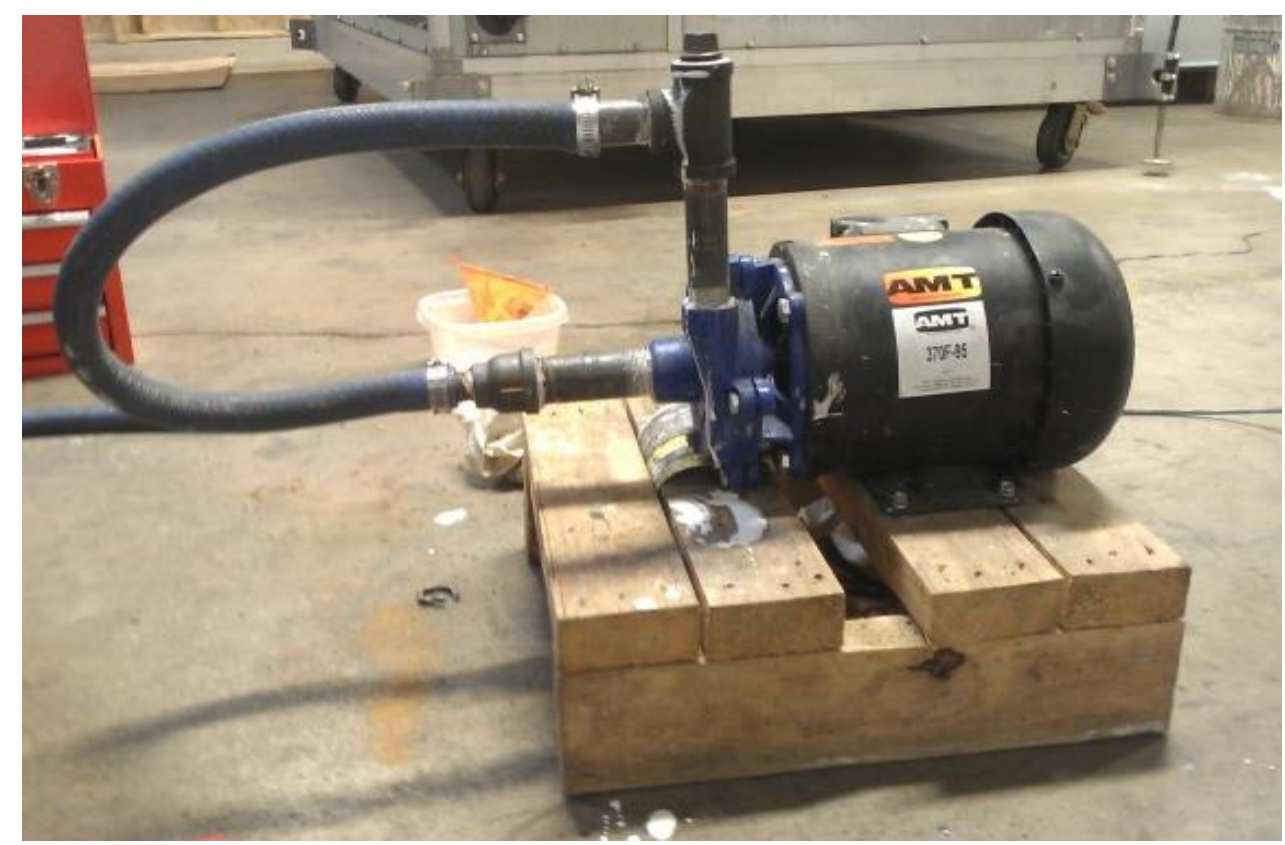

Figure 5.8: Centrifugal Pump Test Setup

The test setup consist a centrifugal pump with on-off switch. The pump was rotated at $2000 \mathrm{rpm}$ to ensure that the slurry is flowing inside the loop. A close loop circuit was made in which mPCS is circulated. Samples are collected at regular time interval and examined under scanning electron microscope for the shell condition of particles.
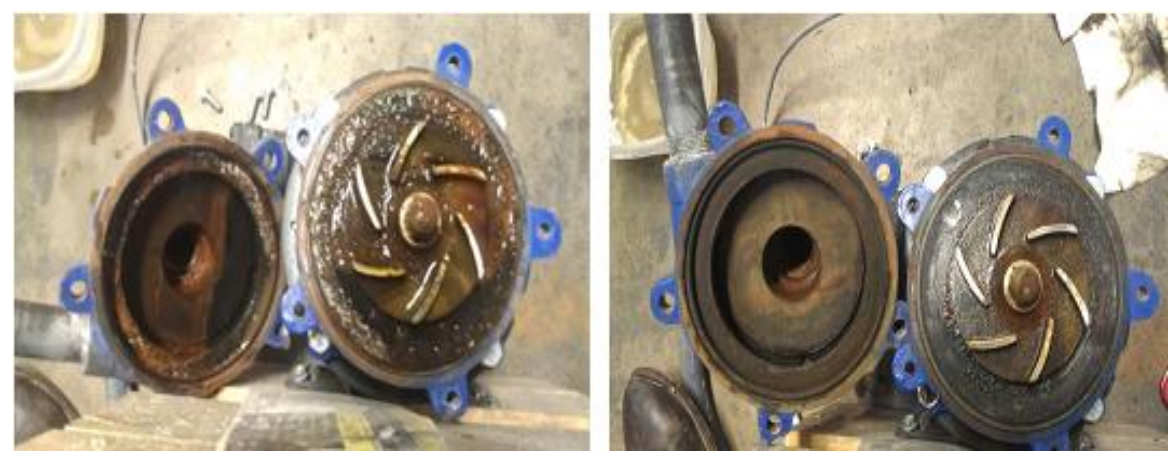

Figure 5.9: Pump Casing before and after cleaning 


\subsubsection{Results}

To check the stability of the slurry capsules after being pumped by centrifugal pump. The slurry samples were collected after certain time intervals and were examined under the SEM. Figure below shows the SEM image of the fresh sample. The average particle size was also measured using in built software with scanning electron microscope. The original particle diameter ranges from $1-3 \mu \mathrm{m}$. The samples were collected and after examining on SEM were measured in its in built software. The average particle size ranges $1.35 \mu \mathrm{m}$.

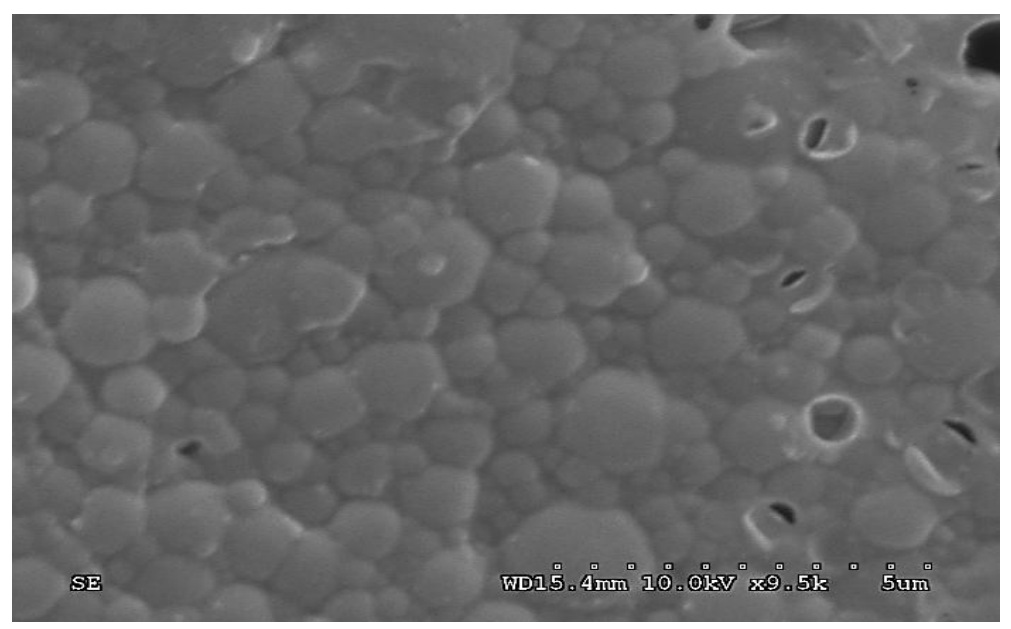

Figure 5.10: SEM image of Fresh mPCS Sample

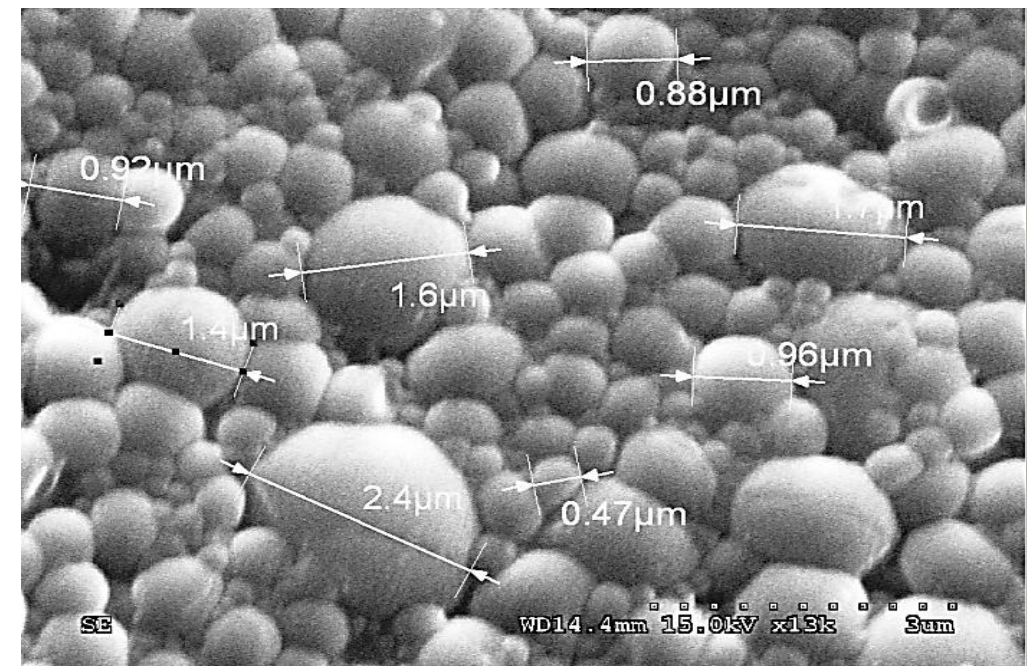

Figure 5.11: Measured Particle Diameter of Fresh mPCS Sample 
After 60 hours of constant running time the first sample was collected and examined under SEM. The pump rpm was set at 2000 to make sure the slurry is flowing. The measured diameters of the particles were $1.095 \mu \mathrm{m}$. The figure below shows the SEM images of the first sample.

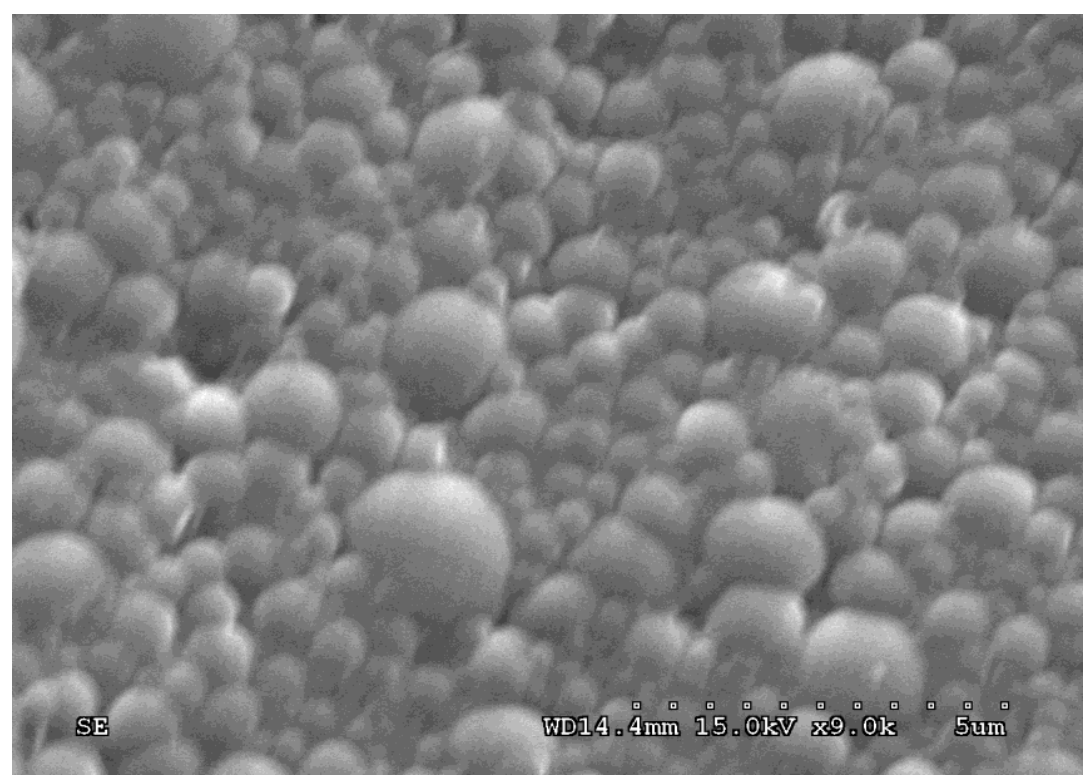

Figure 5.12: SEM Image of Second mPCS Sample

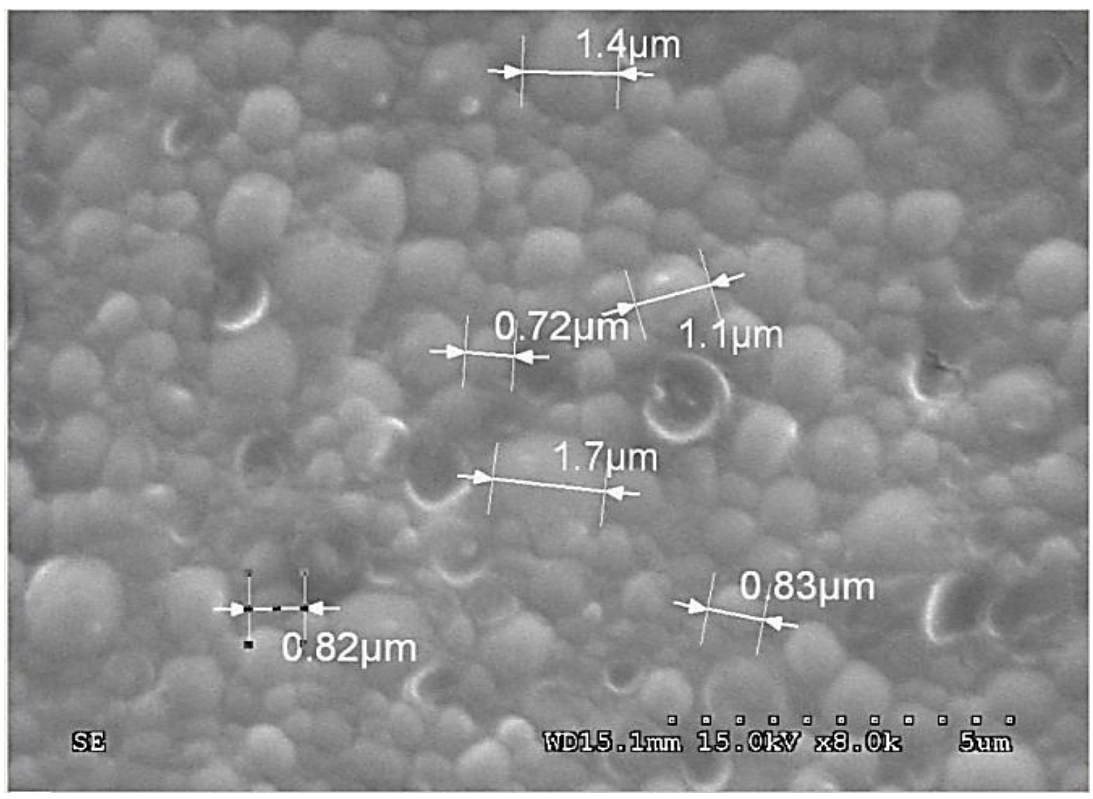

Figure 5.13: Measured Particle Diameter of Second mPCS Sample 
The Image clarity decreases in comparison to fresh sample. The reason being pump has some initial rusting. In addition in order to achieve perfect images more precise procedure needs to be followed. To measure capsules precisely we need a filtration device that separates the liquid phase from the solid phase (unbroken microcapsules) e.g. recommended is a membrane and filter holder

Another sample was collected after $\sim 120$ hours from the first sample. Figure below show the SEM images of the slurry. The pump rpm were maintained at 2000 to keep it flowing. No change in the viscosity of the slurry is observed. The measured diameter of particles were $1.19 \mu \mathrm{m}$. Majority of the capsules looks intact but few shrinkage can be seen if we compare the SEM images with fresh samples.

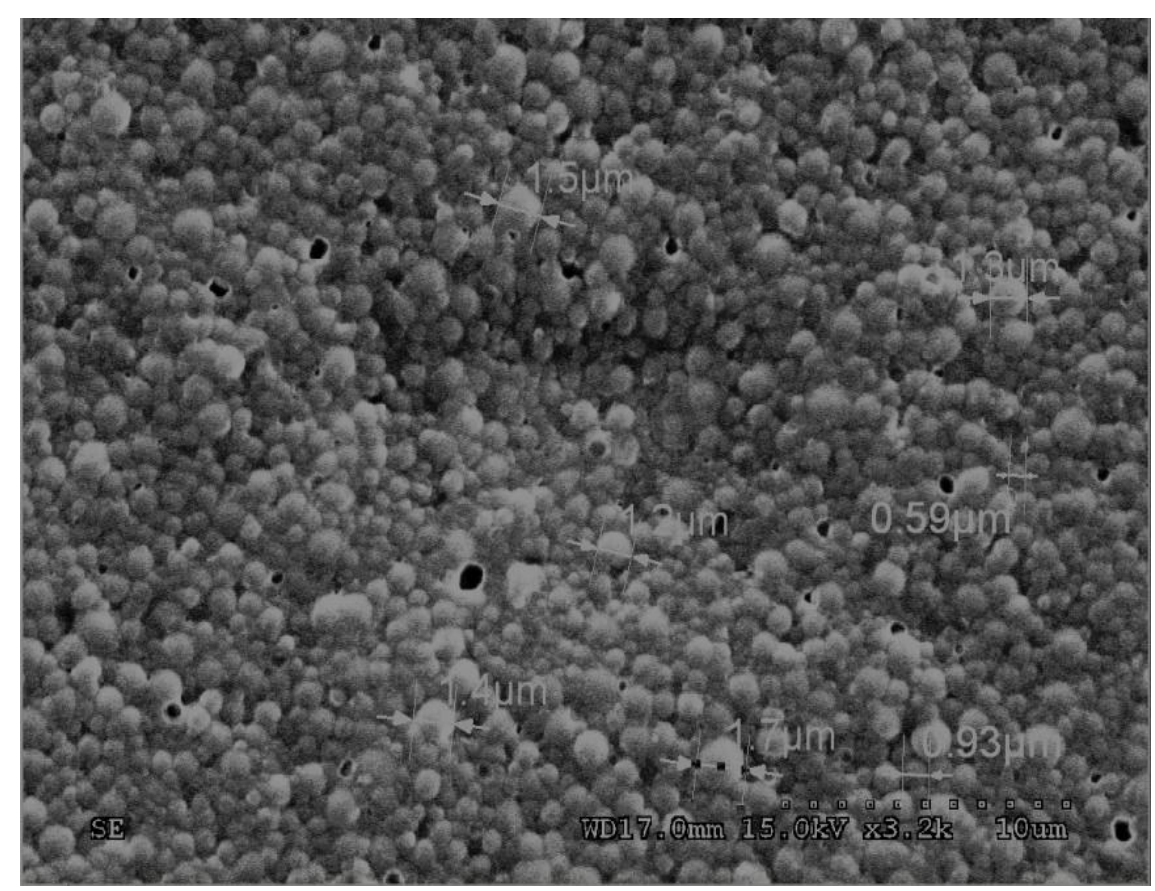

Figure 5.14: SEM Image of Third mPCS Sample 


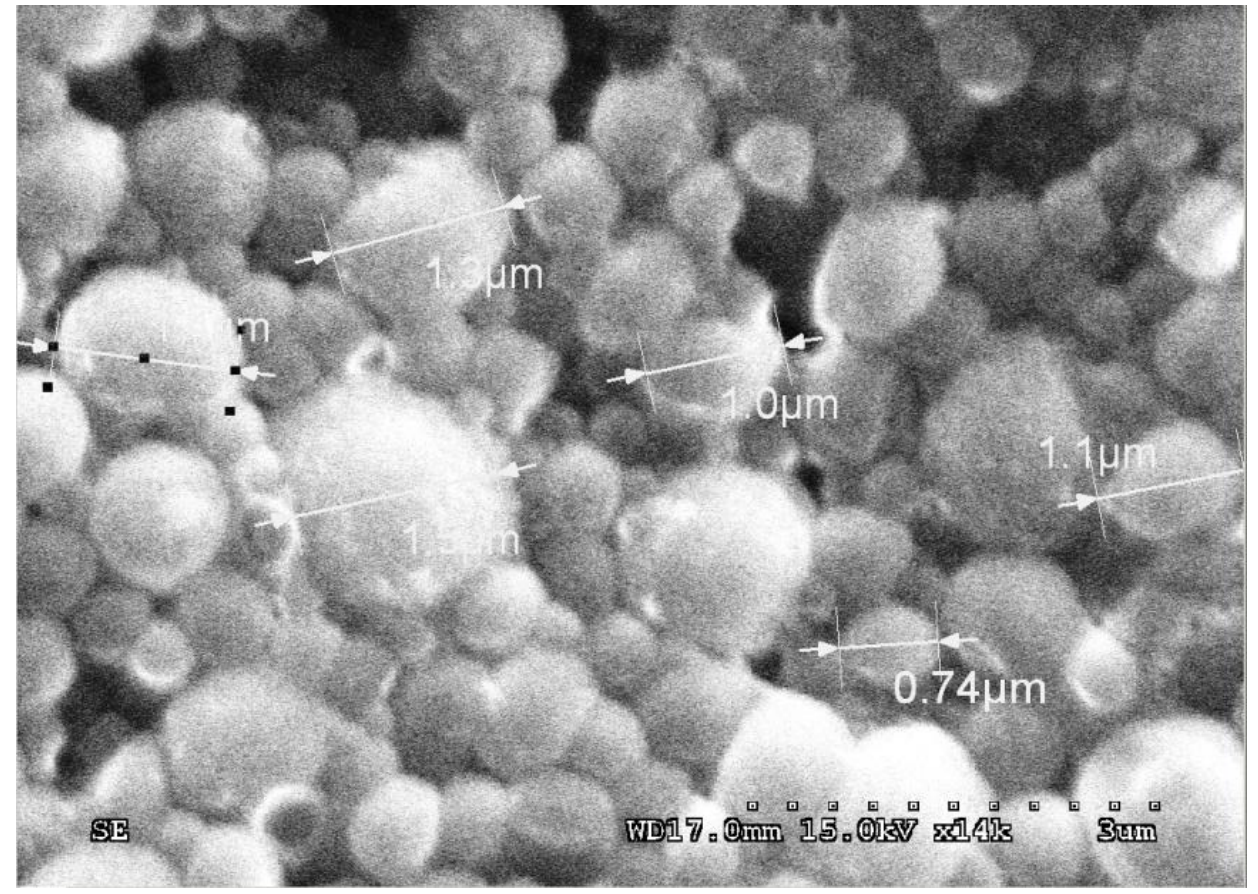

Figure 5.15: Measured Particle Diameter of Third mPCS Sample

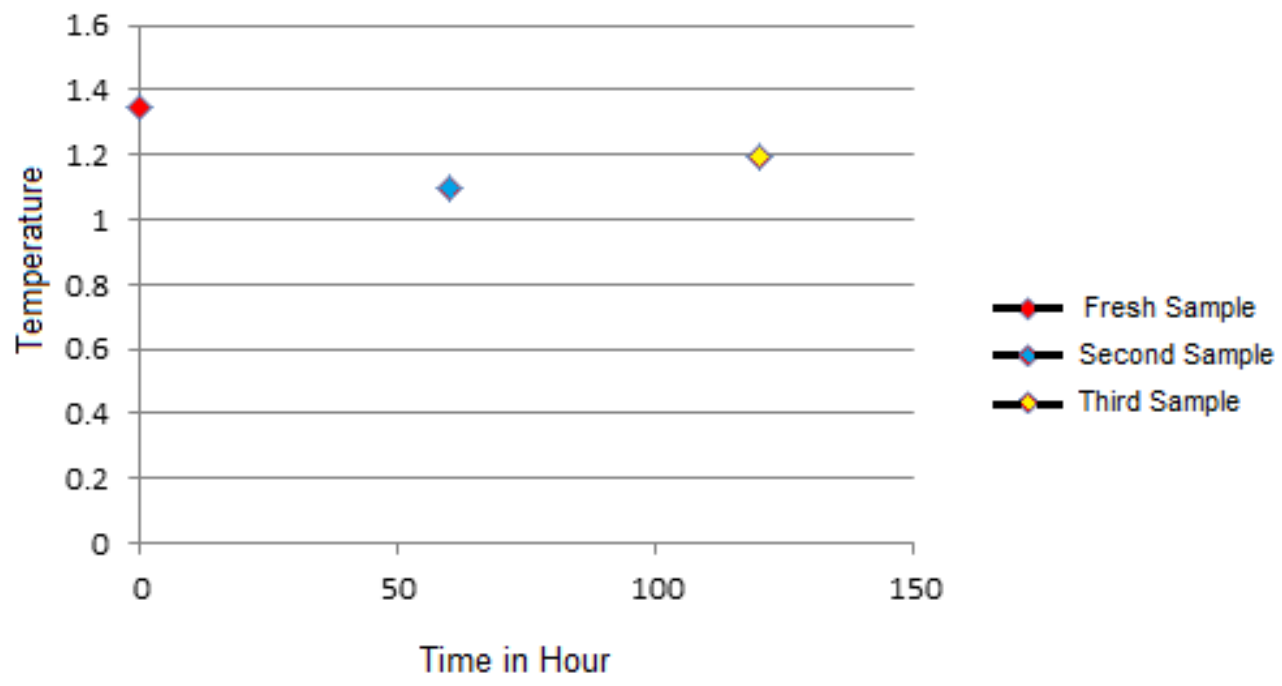

Figure 5.16: Graph for Different Tested mPCS Samples 
Table 5.1: Summary of the Testing for mPCS Samples

\begin{tabular}{|c|c|c|c|c|c|}
\hline $\begin{array}{l}\text { mPCS } \\
\text { Sample }\end{array}$ & $\begin{array}{l}\text { Time period of } \\
\text { Sample }\end{array}$ & $\begin{array}{l}\text { Temp } \\
\text { of } \\
\text { Slurry }\end{array}$ & $\begin{array}{c}\text { Average } \\
\text { Particle } \\
\text { Size } \\
(\mu \mathrm{m})\end{array}$ & $\begin{array}{l}\text { Agglomeration } \\
\text { or Coagulation }\end{array}$ & Remarks \\
\hline 1. & $\begin{array}{c}\text { Tue }- \text { Fri } \\
(2 / 5 / 13-2 / 8 / 13) \\
2.5 \text { days }\end{array}$ & $103^{\circ}$ & 1.095 & No & $\begin{array}{l}\text { Slurry color } \\
\text { turns to brown } \\
\text { ( due to initial } \\
\text { pump rusting) }\end{array}$ \\
\hline 2. & $\begin{array}{c}\text { Fri }- \text { Thurs. } \\
(2 / 8 / 13-2 / 14 / 13) \\
5 \text { days }\end{array}$ & $99^{\circ} \mathrm{F}$ & 1.19 & No & $\begin{array}{l}\text { Slurry color } \\
\text { turns to dark } \\
\text { brown }\end{array}$ \\
\hline 3. & $\begin{array}{c}\text { Thurs. - Fri } \\
(2 / 14 / 13-2 / 22 / 13)\end{array}$ & $96^{\circ} \mathrm{F}$ & 1.07 & No & $\begin{array}{l}\text { Slurry color } \\
\text { turns more dark }\end{array}$ \\
\hline
\end{tabular}

A centrifugal pump has been chosen in accordance with the work of S. Gschwander $r$ et al. [26]. After testing different pumps, S. Gschwander et al. concluded that centrifugal pumps were able to pump the mPCM slurry during a longer period of time without self-destruction or damage to the microcapsule shells. Also, no agglomeration or clogging problems were found during the testing.

\section{$\underline{5.4 \text { Testing for Water vs. mPCS as Thermal Storage Fluid }}$}

The experiment was setup to determine the performance of Micronal $5045-\mathrm{X}$ as thermal storage fluid in comparison with water. A small test rig was constructed which includes, a hot plate, a storage water tank and small cylinder. The figure below shows the schematic of the test setup. 


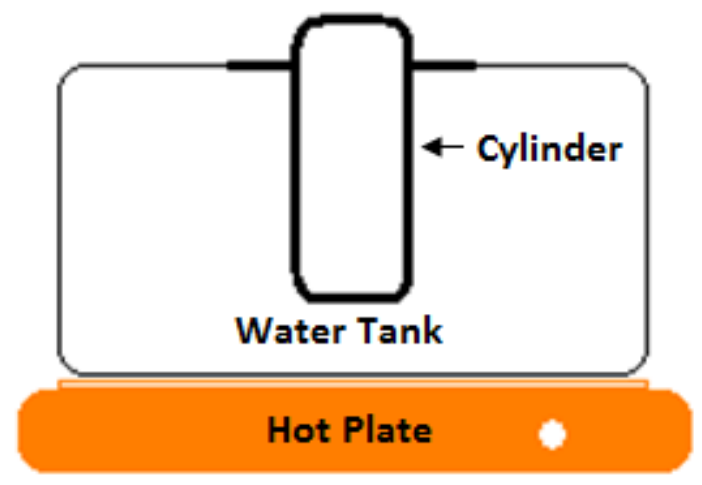

Figure 5.17: Test setup for Thermal storage

Same mass of water and mPCS were poured in the cylinder to obtain the heating curves. In the storage tank water was heated till $140^{\circ} \mathrm{F}$ for both the cases.

Table 5.2: Parameter for the Test Setup

\begin{tabular}{|c|c|c|c|}
\hline Sr. No & Particular & Temperature & Mass in gram \\
\hline 1. & Water storage tank & $\sim 140^{\circ} \mathrm{F}$ & - \\
\hline 2. & Water & $\sim 71^{\circ} \mathrm{F}$ & 200 \\
\hline 3. & Original mPCS & $\sim 71^{\circ} \mathrm{F}$ & 200 \\
\hline
\end{tabular}

\subsubsection{Results}

The temperature range was selected in order to achieve the transition range of the mPCS sample i.e. $72^{\circ} \mathrm{F}-82^{\circ} \mathrm{F}$. The following graphs depicts that it took $\sim 60$ seconds for water sample in cylinder to reach $\sim 103^{\circ} \mathrm{F}$ from $70^{\circ} \mathrm{F}$ with total temperature drop in water storage tank of approximately $2^{\circ} \mathrm{F}$. On the other hand, for mPCS sample it took $\sim 320$ seconds to reach from $\sim 71^{\circ} \mathrm{F}$ to $103^{\circ} \mathrm{F}$ with total temperature drop in tank $\sim 8^{\circ} \mathrm{F}$. 


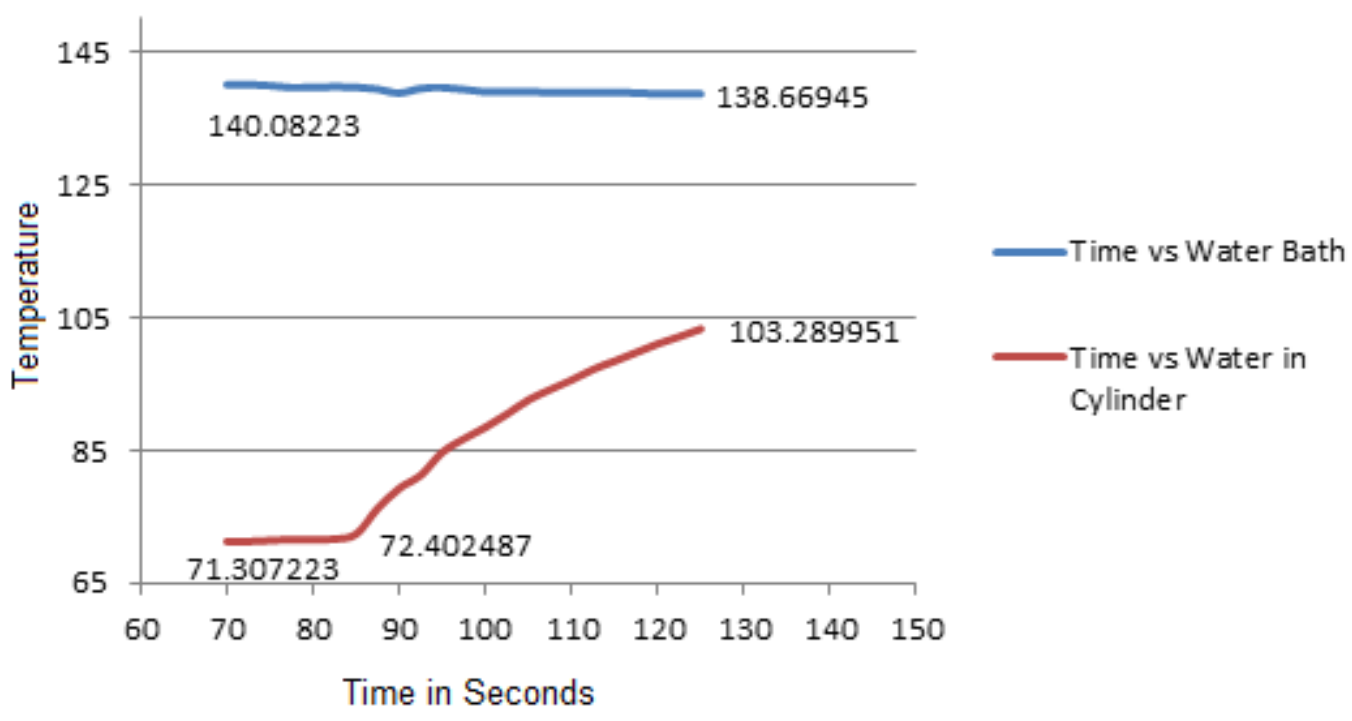

Figure 5.18: Heating Curve for Water

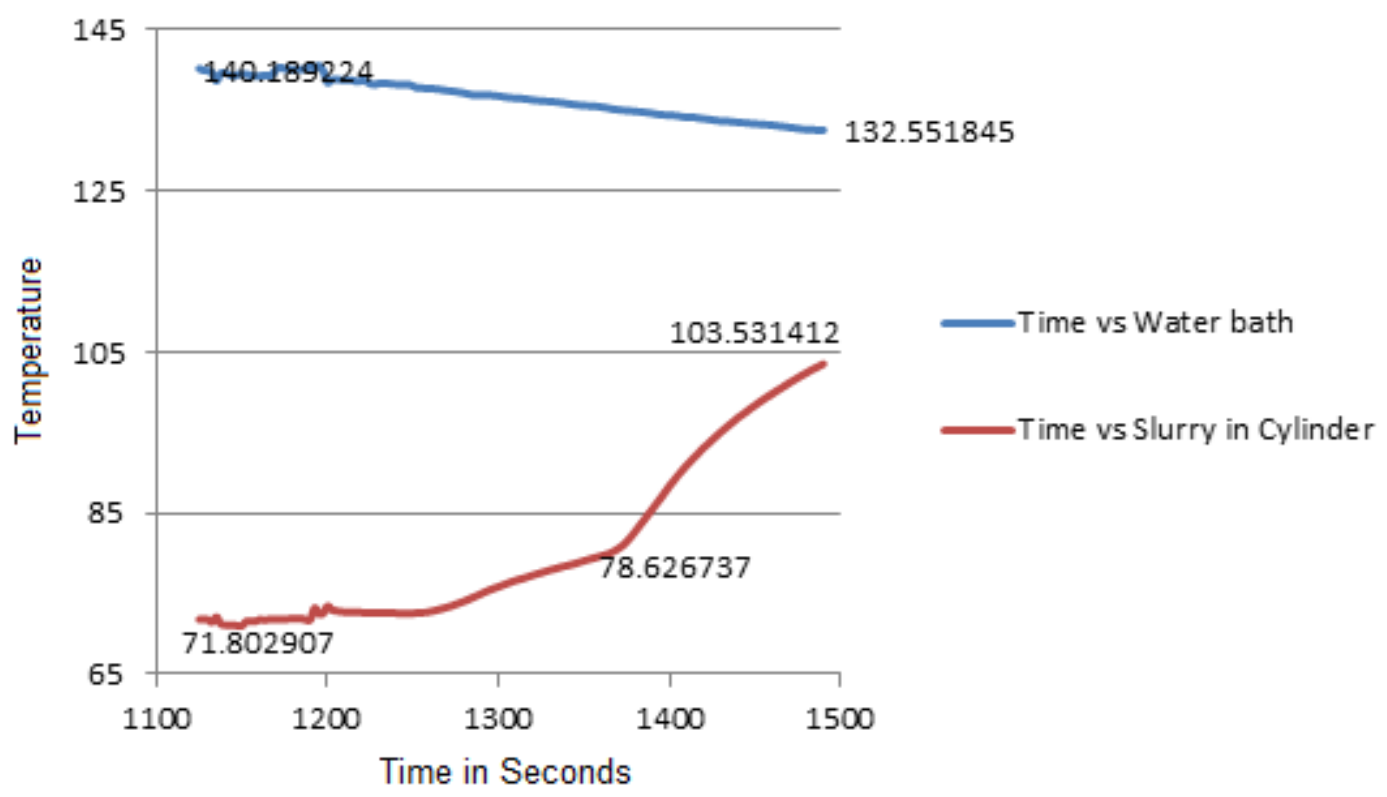

Figure 5.19: Heating Curve for mPCS

Another testing was performed with same setup to figure the performance of mPCS under turbulence. Initial testing was done with water to establish baseline case. Constant heat was added to the system in this case. 


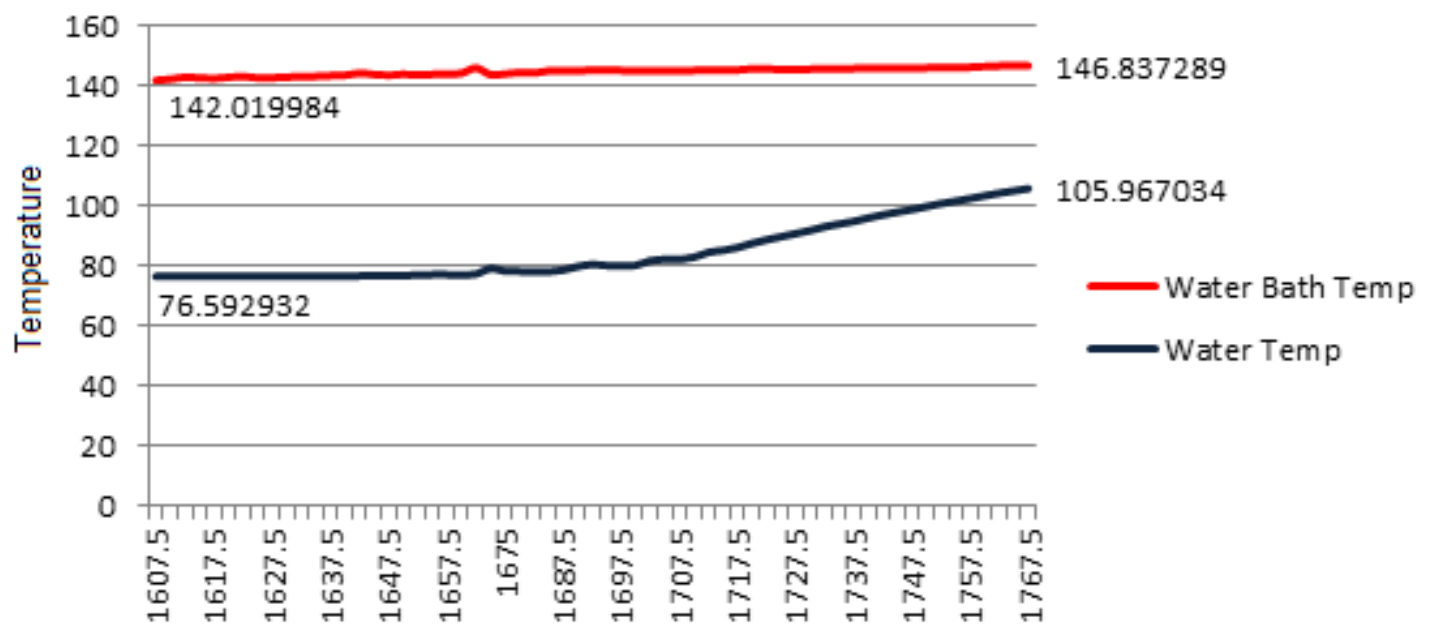

Time in Seconds

Figure 5.20: Water Testing without Stirring

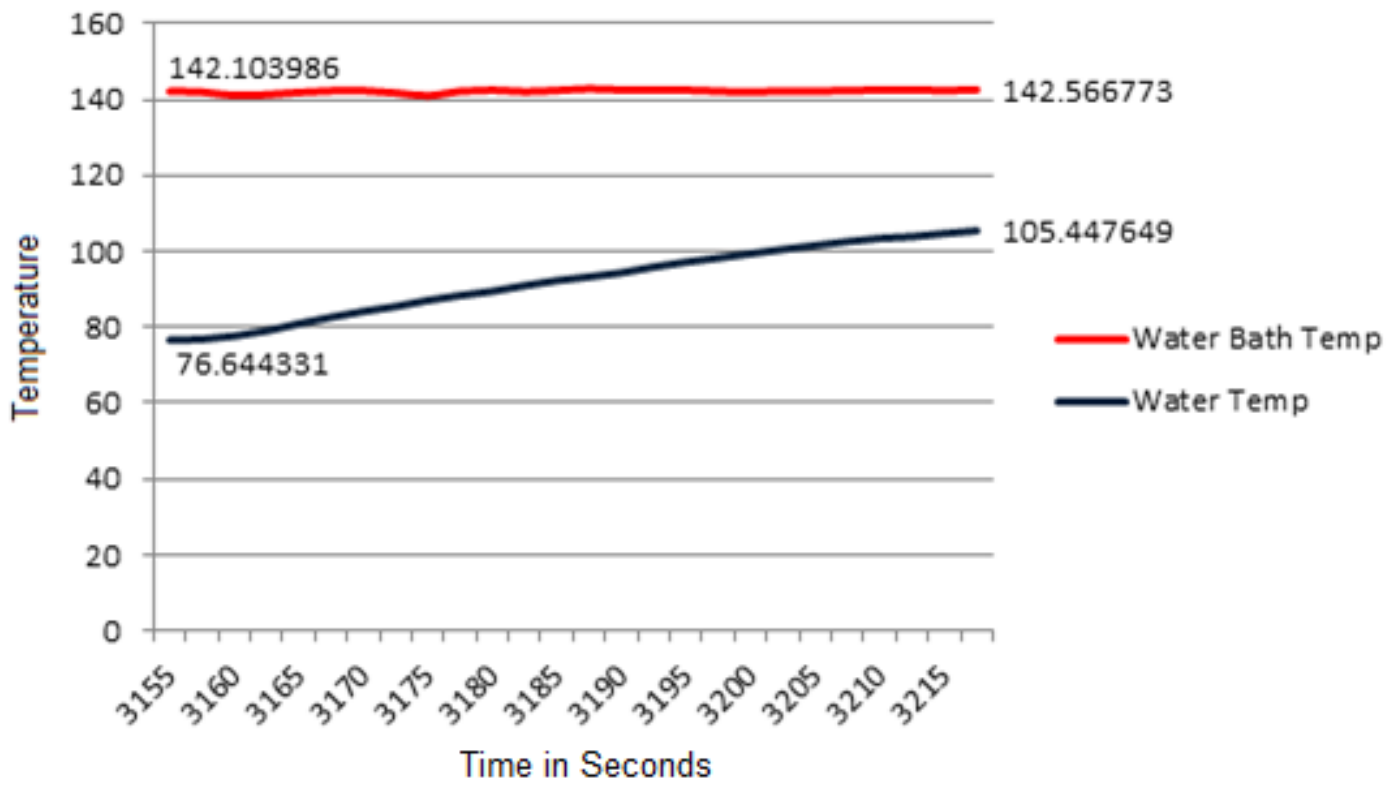

Figure 5.21: Water Testing with Stirring 


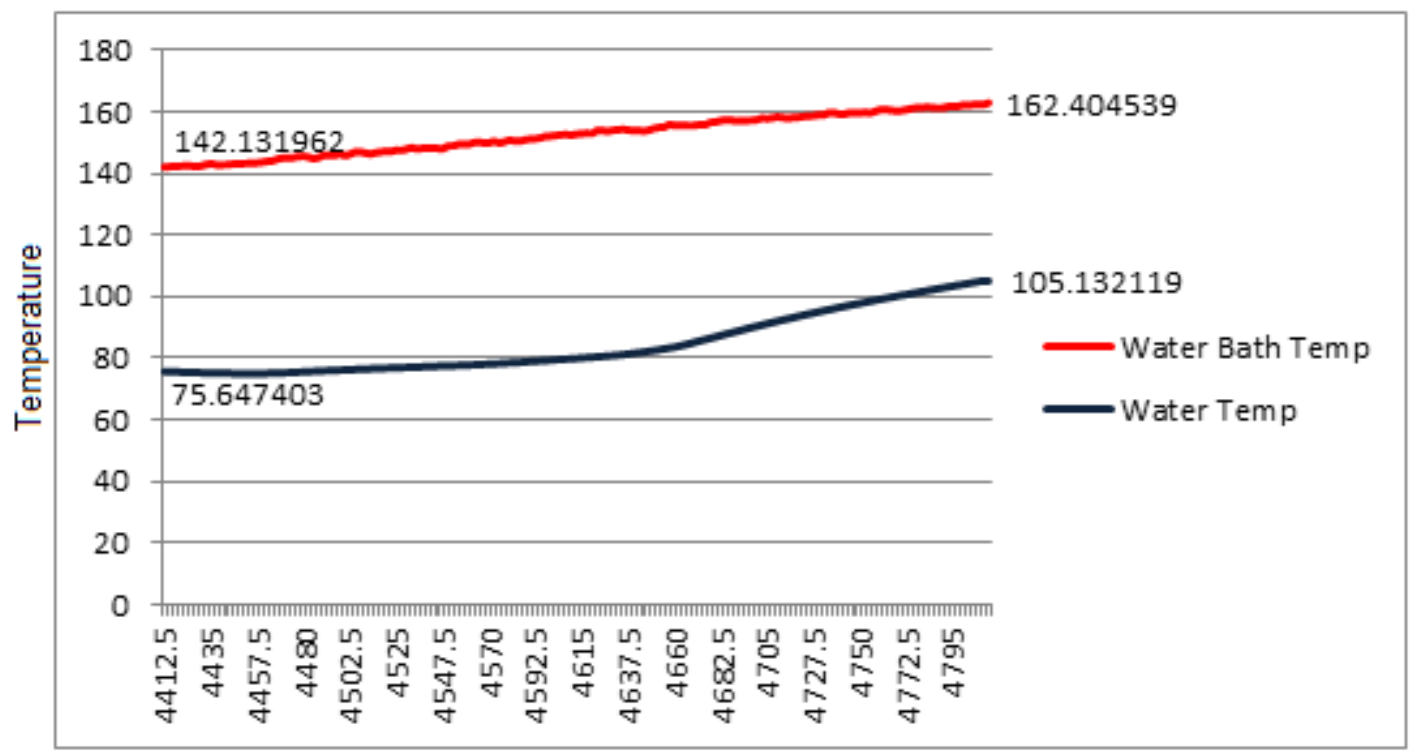

Time in Seconds

Figure 5.22: Slurry Testing without Stirring

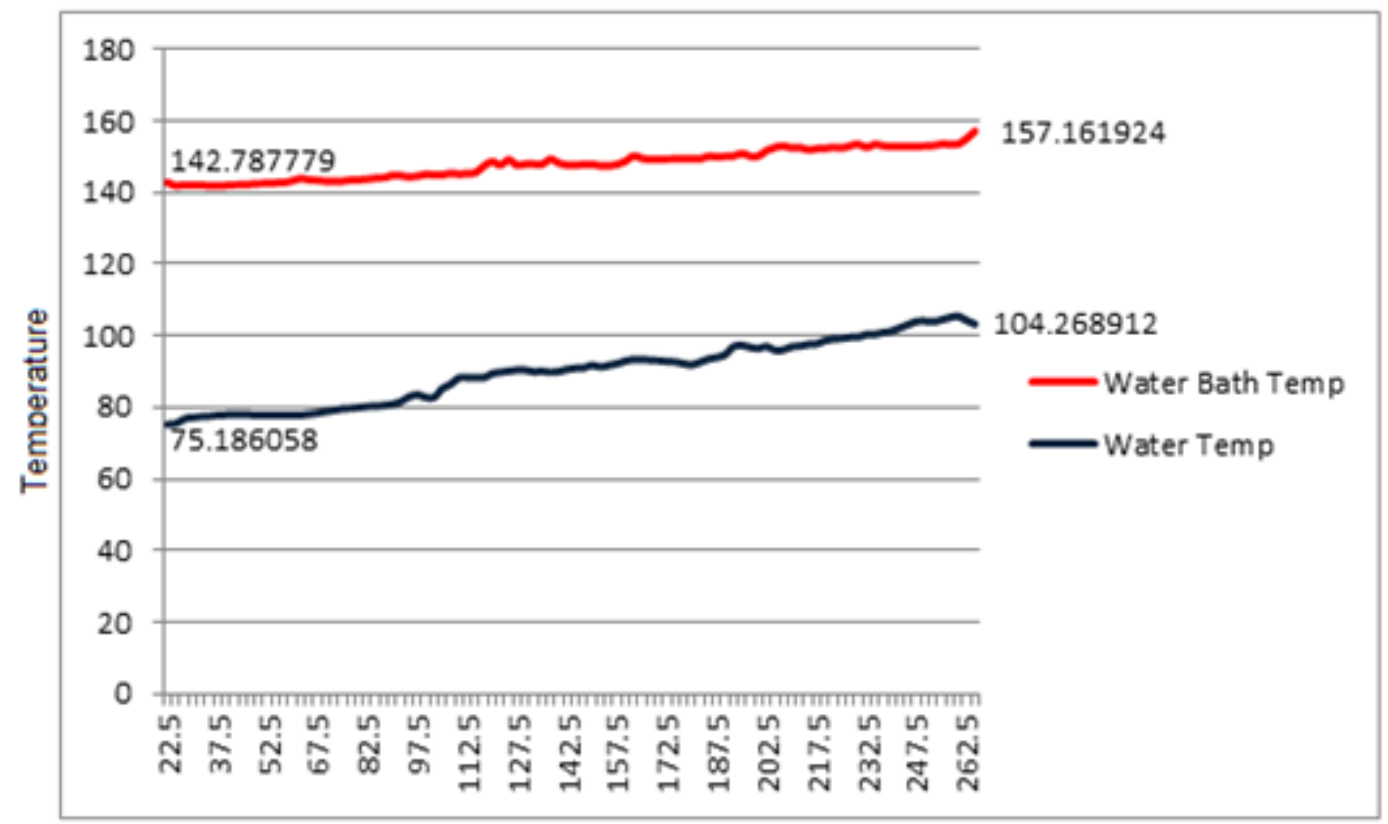

Time in Seconds

Figure 5.23: Slurry Testing with Stirring 


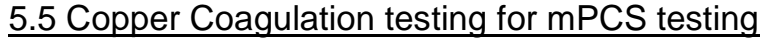

To figure the compatibility of Micronal 5045-X with copper, a closed loop system was constructed. The mPCS was circulated in the system for 15 hours continuously. The slurry was kept stagnant in the system after that for 30 days. The copper loop was inserted in ice bath and centrifugal pump was used to circulate the slurry. Thermocouples were installed at the inlet and outlet of the copper loop when placed in the ice bath to ensure that the slurry is flowing. No, coagulation or agglomeration was found in the system.

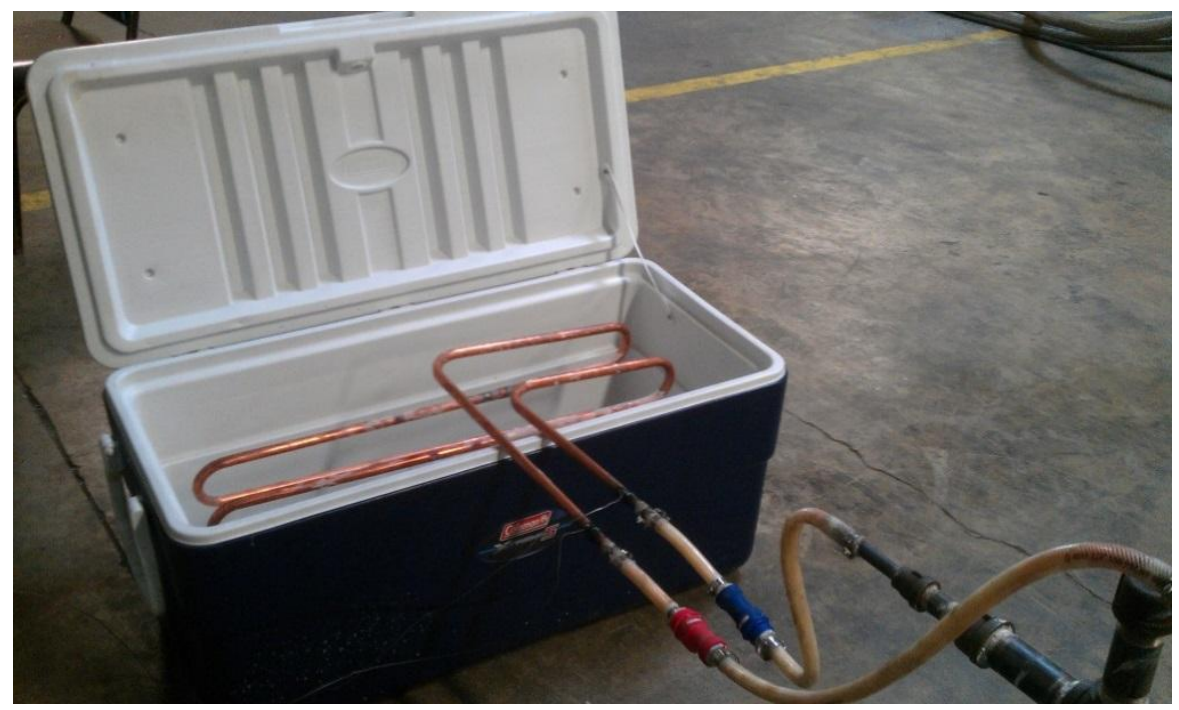

Figure 5.24: Copper Compatibility Test

\section{$\underline{5.6 \text { Final Testing with mPCS slurry }}$}

This new pumpable 5045-X mPCS promise all the possibilities to be used as heat transfer fluid. In addition, small test rig was constructed to see its performance. Shown below is the schematic of the test rig. 


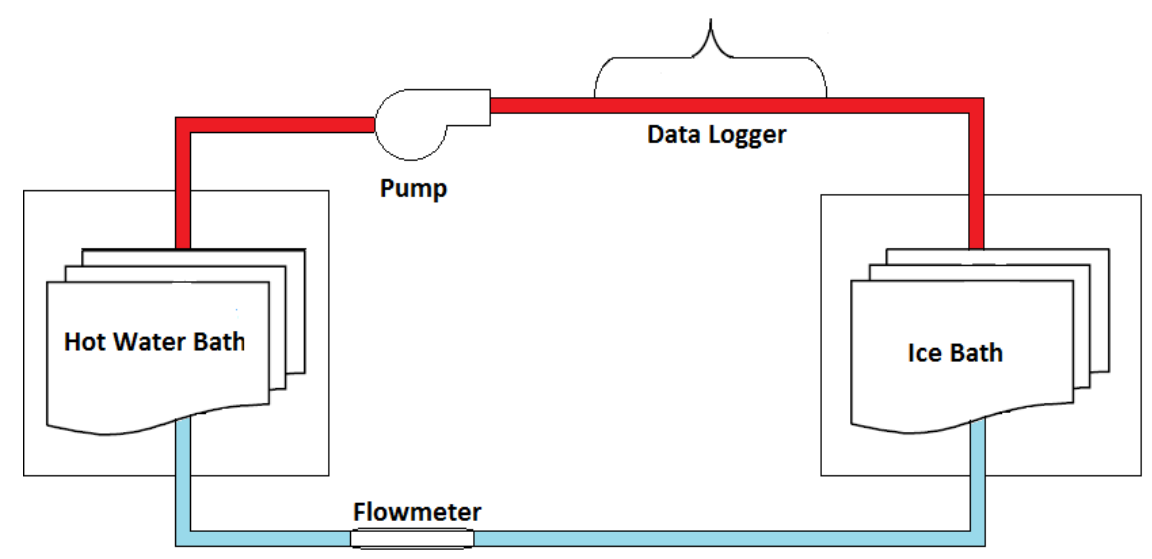

Figure 5.25: Schematic of the Test Rig

It contains two heat exchangers, hot water bath and ice bath. Both heat exchangers contains helical copper coil of helix diameter of $142 \mathrm{~mm}$ with 5 turn. The size of the copper coil was $3 / 8$ " diameter. Both heat exchangers were insulated to reduce the heat loss to surroundings. The heat exchangers were connected using a closed loop circuit. Pump was installed on the hot side of the heat exchanger because cyclic stability is better. Data Logger along with lab VIEW was used to acquire the readings of the system. Thermocouples were installed in the system. Initial testing was done with water. The pump rpm was set to 1000 to ensure its circulating in the loop. The following graph shows the performance with water: 


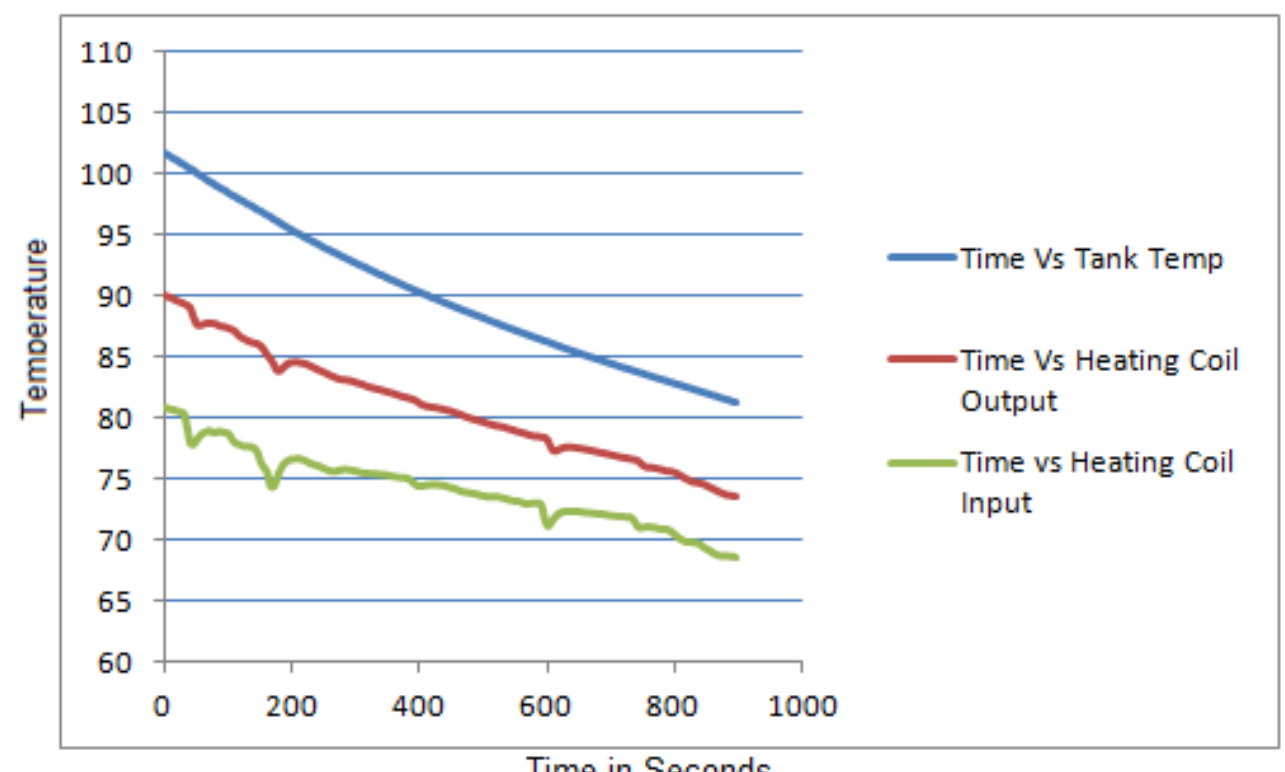

Figure 5.26: Time vs. Temperature Drop for Water

The average temperature drop in heating coil output and heating coil input were around 7 to 8 degrees. The total temperature drop in the tank temperature was from $\sim 101^{\circ} \mathrm{F}$ to $81^{\circ} \mathrm{F}$. The increase in temperature due to pump heating is around $\sim 2$ degrees.

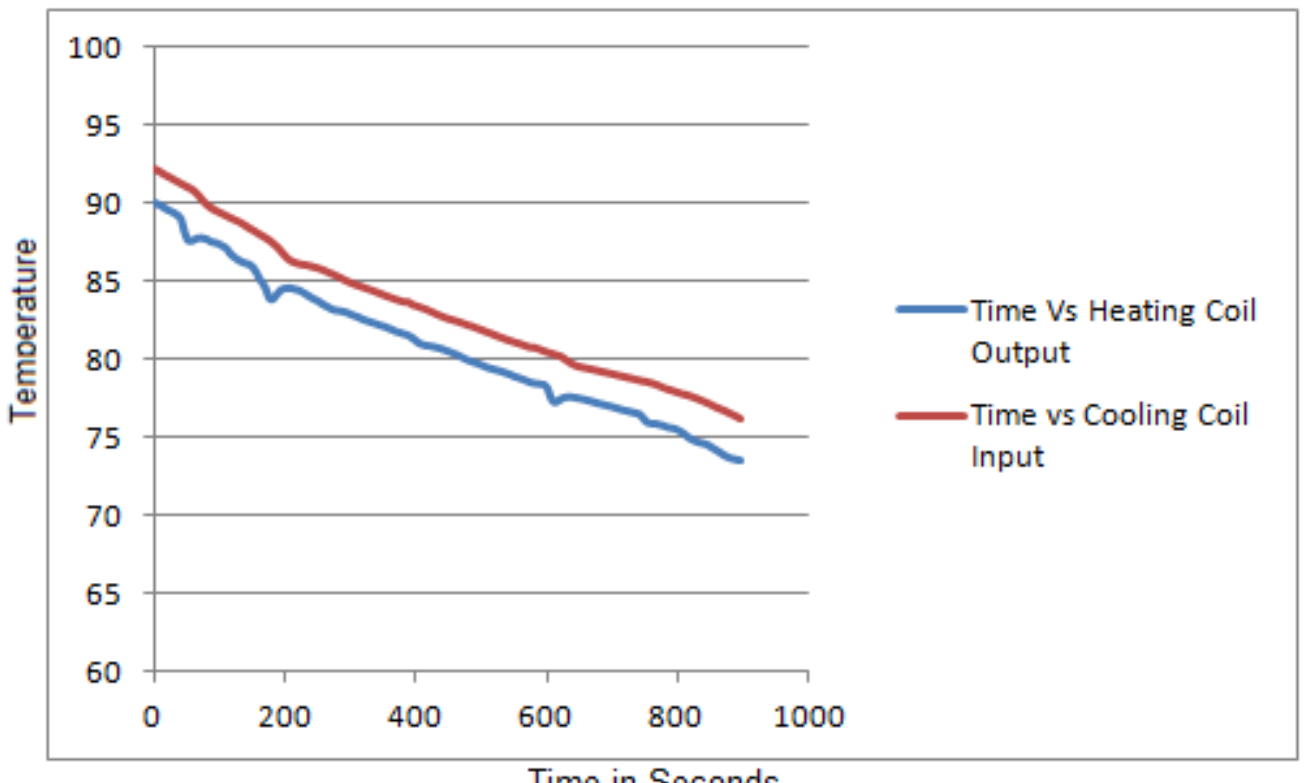

Figure 5.27: Temperature Difference due to Heat from Pump 
The mPCS was circulated in the same test rig after water. The pump rpm were increased to 3600 due to high viscosity of mPCS. In case of mPCS care has taken to ensure that the mPCS attains its transition temperature before entering the heating section and leaving the ice bath section. The temperature of mPCS should be lower than its crystallization point before it enters the heating section and higher than its melting point. This ensures that mPCM particles in slurry changes their phase completely. Following are the graphs for mPCS testing:

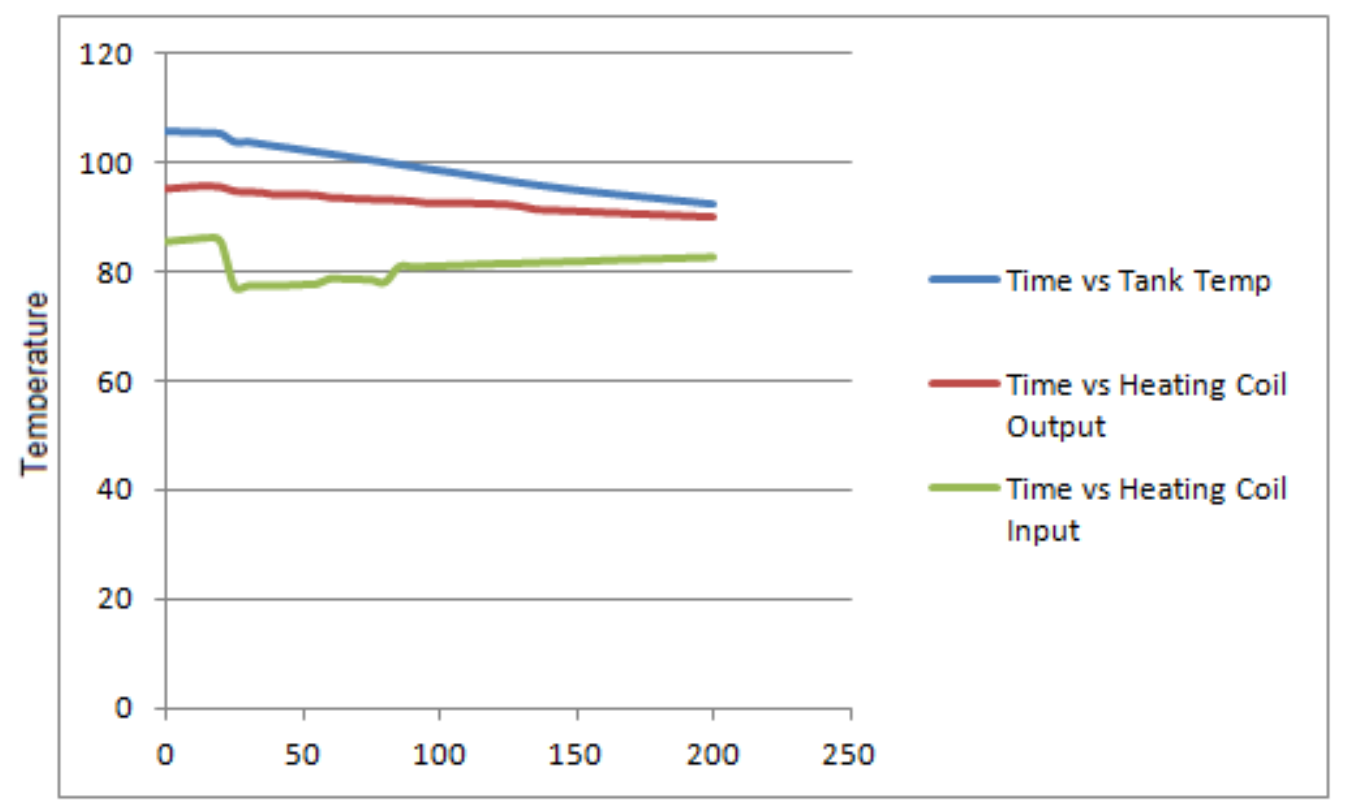

Time in Seconds

Figure 5.28: Time vs. Temperature Drop for mPCS

The average temperature drop in heating coil output and heating coil input remains almost same around 7 to 8 degrees when mPCS was not changing phase. The temperature drop increased to average of 15 degrees from 8 degrees when mPCS was changing its phase completely. The increase in temperature due to pump heating is around $\sim 3$ degrees. 


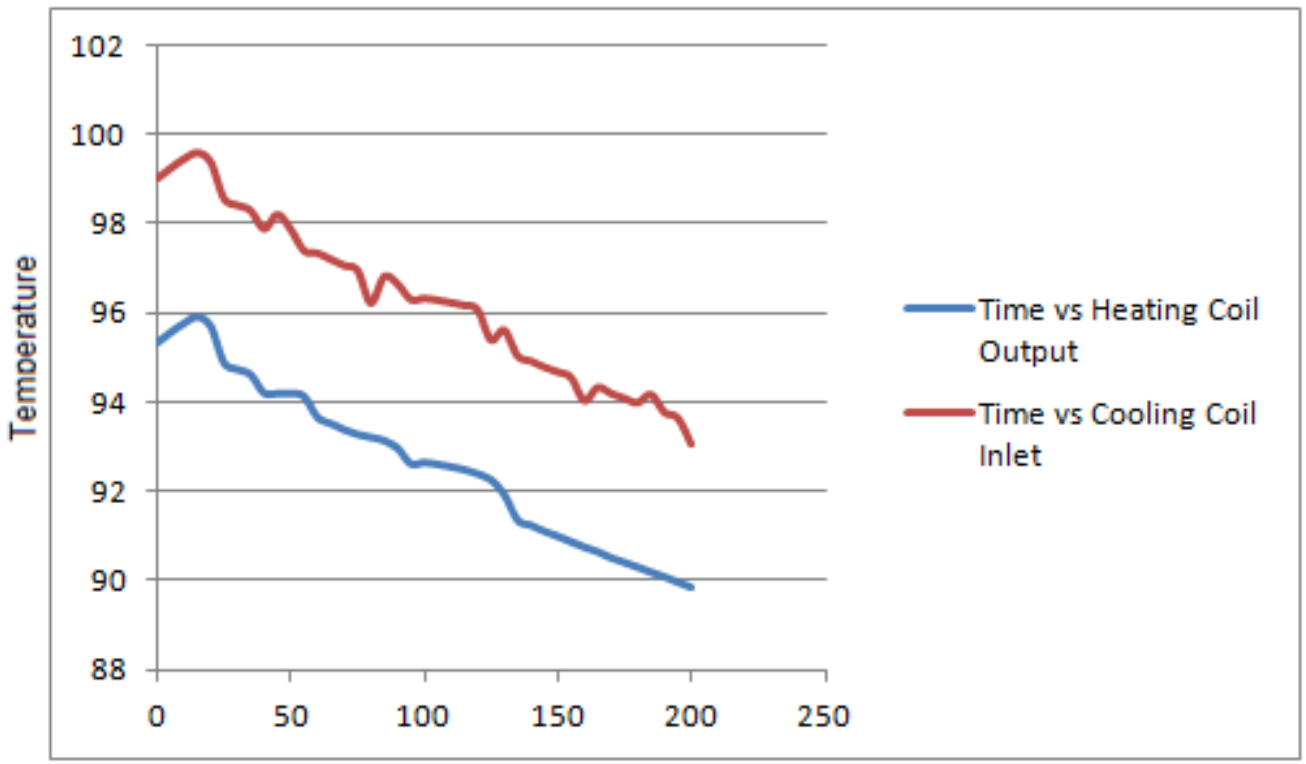

Time in Seconds

Figure 5.29: Temperature Difference due to Heat of Pump

\subsection{Conclusion}

The main objective of this work is to develop standalone pump-able microencapsulated phase change slurry that is able to withstand shear stresses of the pump and other course surfaces of pipe and pipe joints. The conclusions are: Results from scanning electron microscopy depicts that centrifugal pump is able to pump the mPCM particle till $3 \mu \mathrm{m}$ without damaging them. Also, copper is not affecting the mPCS and its not showing any coagulation or agglomeration problem. It can be used as heat exchanger with mPCS. Microencapsulated phase change slurry shows better performance in heat transfer under turbulence. It is true that this mPCS have shown better performance over water in hot water bath case. There is more temperature drop in the water tank. The new Micronal 5045-X mPCS selected promises all possibilities to be used heat transfer fluid. 


\subsection{Future Work}

The mPCS have higher viscosity than water. This tends to increase the pumping power. Experimental analysis will be done with different concentrations of slurry to find out the best heat transfer coefficient with respect to pumping power. Also, experimental analysis will be performed to figure the amount of water usage to lower down the temperature of the slurry below its crystallization point which will be compared with water consumed to cool down the supply air to the same temperature using evaporative cooling technique. 


\section{REFERENCES}

1. Veerendra Mulay., "Analysis of Data Center Cooling Strategies and The Impact of The Dynamic Thermal Management on The Data Center Energy Efficiency", December, 2009.

2. J.D. Mitchell-Jackson Energy needs in an internet economy: a closer look at data centers Energy and resources group University of California, Berkeley

3. Naveen Kannan., "Design and Modeling Techniques for Cooling of Telecommunication Systems", December, 2011.

4. Niket shah., "CFD Analysis of Direct Evaporative Cooling Zone of Air-Side Economizer for Containerized Data Center", May, 2012

5. Inside Sun's Project Black box, http://news.cnet.com/2300-1015 3-6126364.html, October 16, 2009.

6. The Expandable Modular Data Center, "SGI ICE Cube Air", http://www.sgi.com/pdfs/4274.pdf, 2011.

7. Digital Reality Trust, "Power Usage Effectiveness", https://www.digitalrealtytrust.com/pue-efficiency.aspx

8. M. Bramfitt and H. Coles, Lawrence Berkeley National Laboratory, "Modular/Container Data Centers Procurement Guide: Optimizing for Energy Efficiency and Quick Deployment", February 02, 2011.

9. Christian Belady, Andy Rawson, John Pfleuger and Tahir Cader, "Green Grid Data Center Power Efficiency Metrics: PUE and DCIE”, 2008. 
10. Shehabi A., Ganguly S., Traber K., Price H., Horvath A., Nazaroff W. and Gadgil A., "Energy Implications of Economizer Use in California Data Centers", Lawrence Berkley National Laboratory, 2008.

11. Air Side Economizers with Evaporative Cooling, Betsegaw Gebrehiwot, Kushal Aurangabadkar, Nikhil Dhiman, Kasturi Rajagopalan, Gaurang Naware, and Dereje Agonafer University of Texas at Arlington, October 23 - 25, 2012

12. http://www.energystar.gov/index.cfm?c=power mgt.datacenter efficiency economizer airside

13. Shehabi A., Ganguly S., Traber K., Price H., Horvath A., Nazaroff W. and Gadgil A., "Energy Implications of Economizer Use in California Data Centers", Lawrence Berkley National Laboratory, 2008.

14. Syska Hennessy

15. Saket Karajgikar, Veerendra Mulay, Dereje Agonafer, Roger Schmidt, "Cooling of Data Centers using Air Side Economizers", InterPack 2009, California, USA.

16. http://www.intel.com/content/dam/doc/technology-brief/data-center-efficiency-xeonreducing-data-center-cost-with-air-economizer-brief.pdf

17. http://www.splung.com/content/sid/6/page/latentheat

18. Manali Shukla., "Heat Transfer Enhancement by Slurry of Phase Change Material Through Rectangular Porous Channel", December, 2011

19. Atul Sharma, V.V. Tyagi, C.R. Chen, and D. Buddhi. Review on thermal energy storage with phase change materials and applications. Renewable and Sustainable EnergyReviews, 13(2):318-345, February 2009.

20. A.Heinz, W. Streicher. Application of Phase Change Materials and PCM-Slurries for Thermal Energy Storage.

21. Luisa Cabeza, Andreas Heinz, Wolfgang Streicher. Inventory of Phase Change Materials (PCM) 
22. Jorge L. Alvarado, Barclay G. Jones, Charles P. Marsh, David A. Kessler, Chang W. Sohn, Carl A. Feickert, Gary E. Phetteplace, Eric D. Crowley, Ryan J. Franks, and Thomas A. Carlson. [Thermal Performance of Microencapsulated Phase Change Material Slurry, March 2008.

23. E.M. Mechalick and A.T Tweedie. Two component thermal energy storage material. Report NSF/RANN/SE/AER-74-09186, National Science Foundation, Washington, D.C., 1975.

24. http://www.venture-chemical.co.jp/technical/technical leaflet.html

25. Su J-F, Wang X-Y, Wang S-B, Zhao Y-H, Huang Z. Fabrication and properties of microencapsulated-paraffin/gypsum-matrix building materials for thermal energy storage. Energy Convers Manage 2012;55:101-7.

26. S. Gschwander, P. Schossig, and H.-M. Henning. Micro-encapsulated paraffin in phasechange slurries. Solar Energy Materials and Solar Cells, 89(2-3):307-315, November 2005.

27. Syukri Himran, Aryadi Suwono, and G. Ali Mansoori. Characterization of alkanes and paraffin waxes for application as phase change energy storage medium. Energy Sources, Part A: Recovery, Utilization, and Environmental Effects, 16(1):117-128, 1994.

28. Li Huang, Marcus Petermann, and Christian Doetsch. Evaluation of paraffin/water emulsion as a phase change slurry for cooling applications. Energy, 34(9):11451155, September 2009.

29. Xichun Wang, Jianlei Niu, Yi Li, Xin Wang, Binjiao Chen, Ruolang Zeng, Qingwen Song, and Yinping Zhang. Flow and heat transfer behaviors of phase change material slurries in a horizontal circular tube. International Journal of Heat and Mass Transfer, 50(13-14):2480-2491, July 2007. 
30. Ruolang Zeng, Xin Wang, Binjiao Chen, Yinping Zhang, Jianlei Niu, Xichun Wang, and Hongfa Di. Heat transfer characteristics of microencapsulated phase change material slurry in laminar flow under constant heat flux. Applied Energy, 86(12):2661-2670, December 2009.

31. Xianxu Hu and Yinping Zhang. Novel insight and numerical analysis of convective heat transfer enhancement with microencapsulated phase change material slurries: laminar flow in a circular tube with constant heat flux. International Journal of Heat and Mass Transfer, 45(15):3163-3172, July 2002.

32. P. Charunyakorn, S. Sengupta, and S.K. Roy. Forced convection heat transfer in microencapsulated phase change material slurries: flow in circular ducts. International Journal of Heat and Mass Transfer, 34(3):819-833, March 1991.

33. S.K. Roy and B.L. Avanic. Laminar forced convection heat transfer with phase change material emulsions. International Communications in Heat and Mass Transfer, 24(5):653-662, September 1997.

34. Patterson MK. The effect of data center temperature on energy efficiency In: Thermal and thermo-mechanical phenomena in electronic systems, 2008 ITHERM 2008 11th intersociety conference on Orlando, FL; 2008. p. 1167-74.

35. J.D. Mitchell-Jackson Energy needs in an internet economy: a closer look at data centers Energy and resources group University of California, Berkeley (2001

36. J.G. Koomey Worldwide electricity used in data centers. Environ Res Lett, 3 (2008), pp. $1-8$

37. Report to congress on server and data center energy efficiency public law 109-431. US Environmental Protection Agency; 2007 pp. 1-133

38. Brill KG. Moore's law economic meltdown Forbes.com; 2008

39. Brill KG. The invisible crisis in the data center: the economic meltdown of Moore's Law. The Uptime Institute White Paper, The Uptime Institute; 2007 
40. http://www.pumpagents.com/AMTPumps/370F-95.html

41. C.Y. Zhaoa,*, G.H. Zhangb. Review on microencapsulated phase change materials (MEPCMs): Fabrication, haracterization and applications

42. Micronal DS 5045 X, Polymer Dispersions for Construction www.basf.de/dispersions

43. http://www.purdue.edu/rem/rs/sem.htm 


\section{BIOGRAPHICAL INFORMATION}

Nikhil Dhiman received his Bachelor of Technology degree in mechanical engineering at Beant College of Engineering \& Technology, Punjab, India in 2010. During his Bachelor of Technology, He actively participated in many technical activities at national level. Also, he served as a president of the technical society of his college. After obtaining his degree, he worked as $R$ \& $D$ engineer in Sonalika International Tractors Ltd., Hoshiarpur. During his employment with Sonalika International Tractors, he worked on project to develop HydrogenElectric Vehicle. In fall 2011, he began his graduate studies at the University of Texas at Arlington, Texas. While working as a graduate student researcher, he worked on projects related to Evaporative Cooling Technology for large Data Centers. In addition he also completed his thesis in collaboration with industry in Application of Phase Change Materials in Sustainable Cooling of Data Center. During the course of his study, he had an opportunity to collaborate with industrial exposure which helped him to think creatively to bridge the connection between academic knowledge and industry. 